\title{
UICN
}

Valeur économique des services écosystémiques

du Parc National

de l'Ichkeul, Tunisie

Hamed Daly-Hassen 
Valeur économique des services écosystémiques du Parc National de l'Ichkeul, Tunisie 


\section{Valeur économique des services écosystémiques du Parc National de l'Ichkeul, Tunisie}

Hamed Daly-Hassen

L'UICN est une union de Membres composée de gouvernements et d'organisations de la société civile. Elle compte avec l'expérience, les ressources et le poids de ses plus de 300 organisations Membres et les competences de plus de 16000 experts. LUICN fait aujourd hui autorite au niveau international sur l'etat de la nature et des ressources naturelles dans le monde et sur les mesures pour les préserver.

Le Centre de Coopération pour la Méditerranée de l'UICN a ouvert en 2001 avec le soutien du Ministère de l'Environnement espagnol, de La Junta de Andalucia et de l'Agence Espagnole de Coopération Internationale pour le Développement (AECID). Au cours de ses 15 années d'existence, I mission du Centre a ete $d$ influencer, encourager et aider 
La terminologie géographique employée dans cet ouvrage, de même que sa présentation, ne sont en aucune manière l'expression d'une opinion quelconque de la part de l'UICN ou des autres organisations concernées sur lo statut juridique ou l'autorité de quelque pays, teritoire ou région que ce soit, ou sur la délimitation de ses frontières.

Les opinions exprimées dans cette publication ne reflètent pas necessairement celles de l'UICN ou des autres organisations concernées.

Le présent ouvrage a pu être publié grâce à un soutien financier du Fonds de Partenariat pour les Écosystèmes Critiques (CEPF) et de la Fondation MAVA.

Auteur : Dr. Hamed Daly-Hassen

Publié par : UICN, Gland, Suisse et Malaga, Espagne

Droits d'auteur : ๑) 2017 Union internationale pour la conservation de la nature et de ses ressources.

La reproduction de cette publication à des fins non commerciales, notamment éducatives, est permise sans autorisation écrite préalable du détenteur des droits d'auteur à condition que la source soit dûment citée.

a reproduction de cette publication à des fins commerciales, notamment en vue de la vente, est interdite sans autorisation écrite préalable du détenteur des droits d'auteur.

Citation : Daly-Hassen, H. (2017). Valeurs économiques des services écosystémiques du parc National do Mchkeul, Tunisio. Gland, Sulsse Malaga, Espagne. UICN. 104pp.

Reviseurs : Dr. Joël Houdet et Justine Delangue (Membres de la Commission de Gestion des Écosystèmes de l'UICN)

\section{Responsable de l'étude : Maher Mahjoub}

BBN : 978-2-8317-1841-5

DOI: 10.2305/UCN.CH.2017.03.fr

Photo couverture : $\odot$ UICN/Irene Morel

Mise en page : miniestudio.es

Disponible auprès de :

Centre de Coopération pour la Méditerranée

Calle Marie Curie, 22

29590 Campanillas

Málaga, España

Tel.: + 34952028430

Fax: +3495202814

uicnmed@iucn.org

http://www.iucn.org/resources/publications

\section{Table des matières}

\section{Préambule}

\section{Liste des abréviations}

Résumé exécutif

1. Introduction

1.1. Présentation du Parc National de l'Ichkeul

1.2. Objectifs de l'étude

2. Approche méthodologique

2.1. Méthodologie d'analyse

2.2. Classification des biens et services des écosystèmes

2.3. Concept de la valeur économique totale des écosystèmes

2.4. Méthodes d'évaluation de la valeur économique des bénéfices

2.5. Distribution des bénéfices

2.6. Collecte des données

3. Identification des services écosystémiques

fournis par le parc national de l'Ichkeul

3.1. Identification des bénéfices économiques directs pour la population locale et pour les autres usagers

3.2. Inventaire des principales espèces animales et végétales directement utilisées par la population locale, les types d'usage et de collecte

3.3. Identification des bénéfices économiques indirects fournis par le PN

3.4. Identification des bénéfices potentiels

3.5. Liens entre l'état de l'écosystème et les activités pratiquées

3.5.1. Richesse faunistique et écotourisme

3.5.2. Richesse floristique et pâturage

3.5.3. Apports d'eau douce et production de poissons

4. Evaluation économique des services écosystémiques

4.1. Valeurs économiques des bénéfices directs

4.2. Valeurs économiques des bénéfices indirects fournis par le PNI

5. Valeur économique agrégée des services

écosystémiques et leur distribution par bénéficiaire

5.1. Valeur économique agrégée

5.2. Comparaison avec les études antérieures sur le Parc Ichkeul

5.3. Comparaison internationale des valeurs monétaires des services fournis par les zones humides intérieures végétalisées

9 . 
6. Evaluation économique de la conservation des habitats

et de la biodiversité du Parc National de l'Ichkeul

6.1. Principaux facteurs influençant l'équilibre de l'écosystème

6.2. Résultats de l'enquête auprès de la population locale et des visiteurs

6.3. Résultats de l'enquête auprès du public

6.4. Valeur économique de la conservation des habitats et de la biodiversité

7. Evaluation économique des programmes de réhabilitation du parc

8. Conclusions

\section{Références}

Annexe 1 : Questionnaire auprès de la population locale (chefs de ménage)

Annexe 2 : Inventaire des plantes à vertu médicinale

Annexe 3 : Questionnaire pour l'estimation de la valeur récréative du Parc National d'Ichkeu

Annexe 4: Questionnaire sur la valeur de l'amélioration de la biodiversité du Parc National de l'chkeul Tunisie https://goo.gl/kddm7B

Annexe 5 : Actions réaliséesdans le cadre du projet GAP (2005-2009)
Les Zones Clés pour la Biodiversité (ZCB) et les aires proté gées en général, établies pour la conservation de la diversitê biologique et des processus écologiques, sont reconnues comme etant un outil essentiel pour lutter contre la perte de la diversité biologique mondiale. Ces dernières sont sujettes à diverses contraintes mettant en péril la résilience de leurs écosystèmes' et la fonctionnalité de leurs services écosystémiques². Outre les contraintes causées par le de veloppement et la pression anthropique, le constat actue montre qu'l y a un manque d'information sur les valeurs écologiques, sociales et économiques des ZCB, ansi que sur lour contribution pour le développement connunaule le bien-être des populations.

Dans le but de contribuer à l'amélioration des connaissances et des outils de gestion et d'aménagement des Zones Clés pour la Biodiversité, le Centre de Coopération pour la Méditerranée de l'Union Internationale pour la Conservation de Nature (UICN-Med) a bénéficié d'un financement du Fonds de Partenariat pour les Écosystèmes Critiques (CEPF) pour a mire en

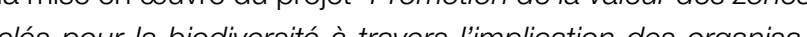
cles pour la biodiversite a travers limplication des organisafions de la societé civile dans leur conservation et gestion en Afrique du Nord. Cest dans ce cadre que sinscrit la pré sente étude qui porte sur l'évaluation économique des services écosystémiques du Parc National de l'Ichkeul en Tunisie.

La mise en œuvre de ce projet vise l'amélioration de conservation et du statut de protection d'un certain nombre de ZCB, en Algérie, en Libye, au Maroc et en Tunisie. En Tunisie, le Parc National de l'Ichkeul (PNI), faisant partie des ZCB retenues par ce projet, a été choisi comme zone d'étude. L'objectif étant de développer une évaluation de valeur économique des services écosystémiques rendus par PNI. Cette évaluation permettra de renforcer les connaissances sur ledit site et de sensibiliser davantage les dec- deurs et les usagers pour assurer une conservation et une gestion durable de son patrimoine naturel et culture.

Cette étude a été réalisée sous la supervision de Monsieur Maher Mahjoub (Coordinateur du programme Afrique du Nord à l'IUCN-Med), de Monsieur Habib Abid (Directeur de Conservation de la Nature, DGF) et de Mme Nabiha Ben Mbarek (Responsable de l'Unité chargée du suivi de l'Ichkeul à l'ANPE).

Différentes institutions ont joué un rôle primordial par leurs conseils et ont collaboré très étroitement pour fournir les données nécessaires : DGF (Mr Youssef Saadani, Mme Hela Guidara, Mr Jamel Tahri), ANPE (Mr Mahmoud Doggi, Mme Yoldiz Kassaoui, Mme Marie-José ElDumi), DG Péche Mr Rafik Nouall, Mr Mehrez Besita), DGACTA (Mr Issam AnaPr, Mr Stheddine Ghechou), CRDA Bze (Mr IMr ADdessatar Belkou Mne Kou), CRDA Bizerte (Mr Abdessaah) OID Ghezla Kar Mohazed Tanar Nouri, Mene Nada ho, OTD G Eza (Mr Mohamed Tahar Noun, Mme Nad a Bouakline), DG Barrages (Mr Hassen Ben Ali, Mr Mehrez Rejeb), SONEDE (Mr Montasar Chebili, Mr Mohamed Hassan Baouab, Mme Rim Oueslati), CTV Mateur (Mr Mohamed Hedi Ghanmi), CRDA Mateur (Mr Taoufiq Jaziri), INAT (Mr Mohamed Salah Romdhane), WWF (Mr Faouzi Maamoun, Mr Sami Ben Achour), Association Les Amis des Oiseaux (Mme Claudia Feltrup-Azafzaf). Aussi, Mr Habib Ghazouan Melle Mariem Khalfaoui et Mr Mokhtar Aloui ont contribue à encuêtes et la collecte des données. Certaines de ces institutions et d'autres, ainsi que Mr Boubaker Houman, Profsseur Universitire, Mr Joet Houdet, expert membre de la commission de gestion des écosystimes de "UICN Mme Justin Delangue exper en servistes ecosyèmiques au Comis assistant au programme Afrique du Nord de l'IUCN, ont aussi revise et commenté la première version de cette étude. Qu'ils soient tous remerciés. 
Liste des abréviations

\begin{tabular}{|c|c|}
\hline$\$ / \epsilon$ & Dollar des Etats-Unis / Euro \\
\hline ANPE & Agence Nationale de Protection de l'Environnement \\
\hline BV & Bassin versant \\
\hline CAP & Consentement à payer \\
\hline CCP & Capacité de charge physique \\
\hline $\mathrm{CCR}$ & Capacité de charge réelle \\
\hline CICES & Classification internationale commune des services écosystémiques \\
\hline CRDA & Commissariat Régional du Développement Agricole \\
\hline CTV & Cellule Territoriale de Vulgarisation \\
\hline DGACTA & Direction Générale de l'Aménagement et de Conservation des Terres Agricoles \\
\hline DGBTH & Direction Générale des Barrages et des Grands Travaux Hydrauliques \\
\hline DGF & Direction Générale des Forêts \\
\hline DG Pêche & Direction Générale de la Pêche \\
\hline DT & Dinar Tunisien \\
\hline GEF & Global Environment Facility (FEM) \\
\hline INAT & Institut National Agronomique de Tunisie \\
\hline INS & Institut National de la Statistique \\
\hline IPBES & Intergovernmental Science-Policy Platform Biodiversity and Ecosystem Services \\
\hline MAB & Programme de l'UNESCO de l'Homme et la Biosphère \\
\hline MARH & Ministère de l'Agriculture et des Ressources Hydrauliques \\
\hline МСT & Méthode de Coût de transport \\
\hline MEA & Millenium Ecosystem Assessment \\
\hline MEC & Méthode d'Evaluation Contingente \\
\hline ONG & Organisation Non Gouvernementale \\
\hline OTD & Office des Terres Domaniales \\
\hline PAG & Plan d'Aménagement et de Gestion \\
\hline PAM & Plantes Aromatiques et Médicinales \\
\hline PDC & Plan de Développement Communautaire \\
\hline PIB & Produit Intérieur Brut \\
\hline $\mathrm{PNI}$ & Parc National de l'Ichkeul \\
\hline Pop. & Population \\
\hline PPA & Parité du pouvoir d'achat (PPA) \\
\hline PSE & Paiements pour les Services Environnementaux \\
\hline REF & Régie d'Exploitation Forestière \\
\hline SECADENORD & Société d'Exploitation du Canal et des Adductions des Eaux du Nord \\
\hline SONEDE & Société Nationale d'Exploitation et de Distribution des Eaux \\
\hline STATA & Data Analysis and Statistical Software \\
\hline STL & Société Tunisie Lagunes \\
\hline $\mathrm{tCO}_{2}$ & Tonne de dioxyde de carbone \\
\hline TEEB & The Economics of Ecosystems and Biodiversity \\
\hline UE & Union Européenne \\
\hline UF & Unité Fourragère \\
\hline UICN & Union Internationale pour la Conservation de la Nature \\
\hline UICN-Med & Centre de Coopération pour la Méditerranée de l'UICN \\
\hline UNESCO & United Nations Educational, Scientific and Cultural Organization \\
\hline VET & Valeur Economique Totale \\
\hline WWF & World Wildlife Fund \\
\hline ZCB & Zones Clés pour la E \\
\hline
\end{tabular}

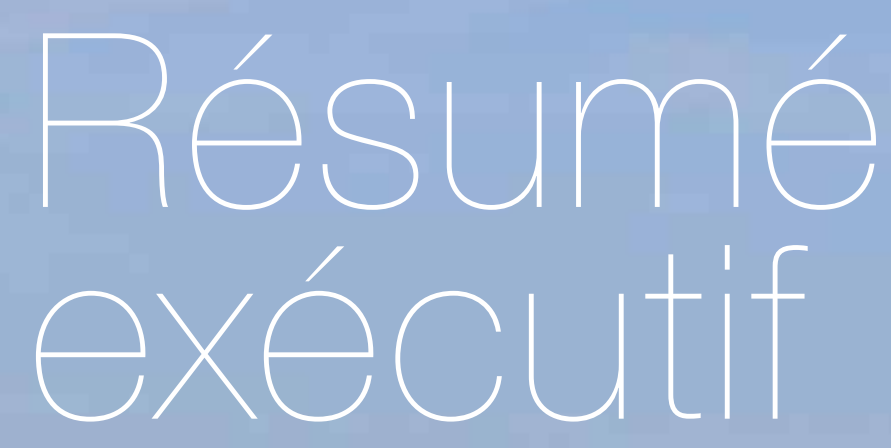

Résumé exécutif

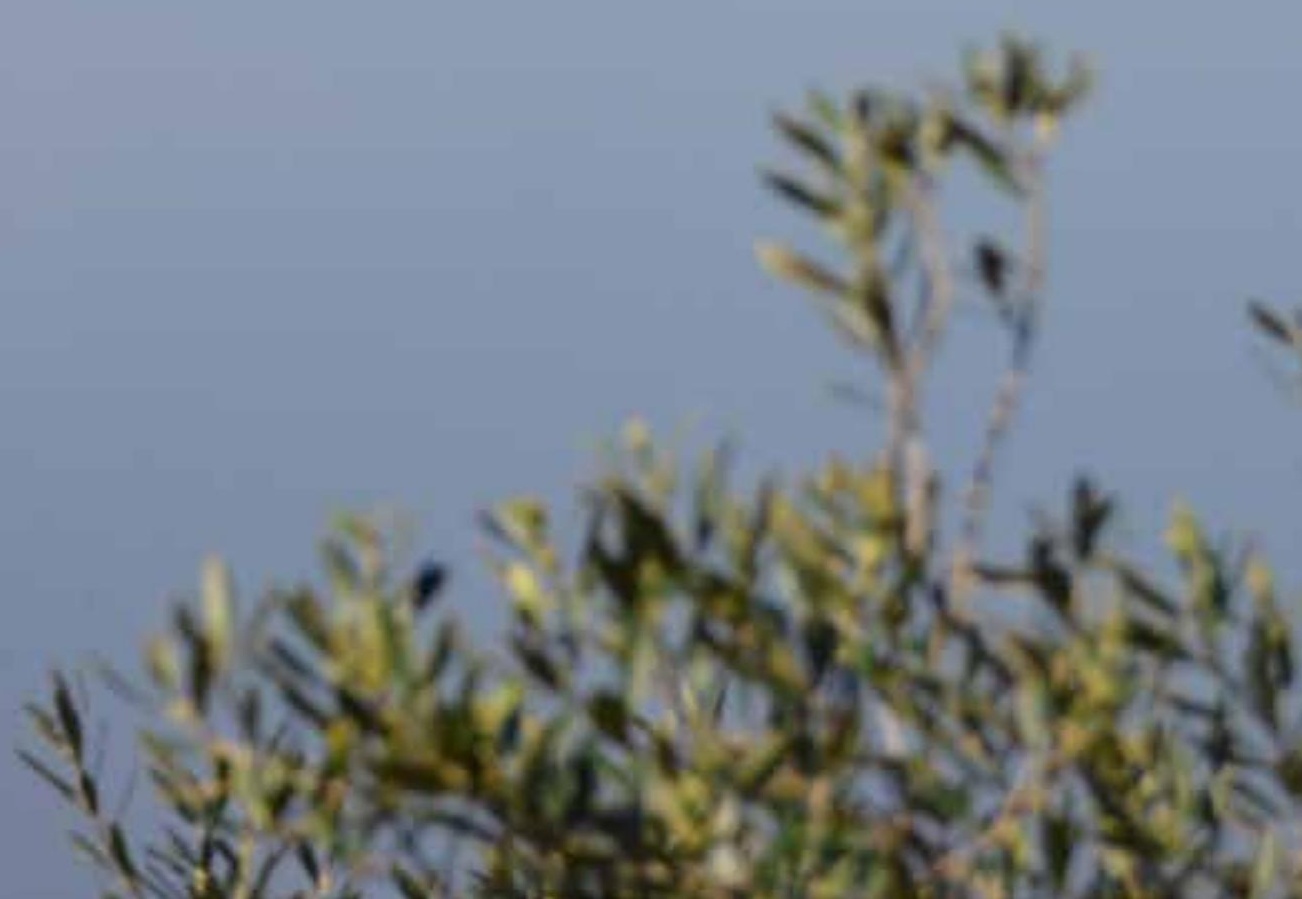




\section{Introduction}

Le Parc National de l'Ichkeul se situe au Nord-Est de la Tunisie à $75 \mathrm{Km}$ de Tunis. II occupe 12.600 ha, couvrant successivement la montagne (1.363 ha), le lac Ichkeul (8.500 ha), et les marécages (2.737 ha). Le lac et les marais sont depuis longtemps reconnus comme l'une des principales zones humides du bassin occidental de la Méditerranée. est aussi connu comme un lieu de refuge pour les oiseaux. Le lac est alimenté en eau douce par un bassin versant d'une superficie de $2080 \mathrm{~km}^{2}$ et est en relation avec la mer par l'intermédiaire du lac de Bizerte via l'Oued Tinja.

L'équilibre de l'écosystème et la préservation de la biodiversité est tributaire de la salinité, dépendante de la quantité d'eau dans le lac (pluviométrie, lâchers des barrages et la gestion du débit dans l'Oued Tinja).

Les principaux enjeux sont relatifs à l'interdiction de certain usages directs (pâturage, pêche) pour la population locale, et la réduction du niveau d'eau dans le lac et la salinisation des eaux. En effet, l'installation des barrages en amont destinés à l'alimentation en eau potable et à l'irrigation a contribué au déséquilibre de l'écosystème.

Cette étude vise à évaluer la valeur économique des services écosystémiques rendus par le parc national de l'Ichkeul (PNI). Cette évaluation permettra de renforcer les connaissances sur le site et de sensibiliser davantage les décideur et les usagers pour assurer la conservation et l'utilisation durable des différentes composantes de la biodiversité. Ceci permettra aussi de concilier les objectifs du PNI avec les attentes et les aspirations de la population locale, notamment en termes d'usage et de valorisation des produits.

\section{Méthodologie}

L'approche méthodologique est composée de cinq étapes : (i) Identifier et classer les services écosystémiques fournis par le PNI selon la classification internationale commune des services écosystémiques (CICES) : services d'approvisionnement, services de régulation et services culturels. (i) Estimer leurs valeurs utilisant différentes méthodes d'év. (ii) tion économique, ainsi que leur distibution entre les ber ciaires. (iii) Etablir les liens entre les qualités écologiques du ciaires. (IIi) Etablir les liens entre les qualités ecologiques dita tive et quantitative des services écosystémiques. (iv) Estimer a valeur économique de l'amélioration de la biodiversité en les ben la menode devaluation contingente. (v) Comparer les bénéfices rendus par le PNI, ainsi que la valeur de l'ameloration de la biodiversité par rapport aux coûts des programmes de préservation du parc, y compris le coût d'une quantité d'eau additionnelle afin d'assurer l'équilibre de l'écosystème en année sèche.

Les données utilisées sont les statistiques officielles et les données fournies par les différentes institutions, les publications scientifiques et les etudes. Aussi, trois enquetes on eté menées auprès de la population résidante locale (100 menages), des visiteurs (93 personnes) et du public (43) aff de collecter les données pour l'application des différentes méthodes choisies.

\section{Valeurs des services}

écosystémiques

a valeur économique agrégée des services écosystémiques est estimée à 6,270 millions $\mathrm{DT}^{3}$ en 2015. La vaeur des services de régulation, ou les bénéfices d'usage indirect, constituent la majorité des bénéfices (73\%), alors que la valeur des bénéfices sur site (services d'approvisionnement (18\%) et culturels (9\%)) ne constitue que $27 \%$ de valeur agrégée. Les valeurs importantes obtenues correspondent aux services de protection contre les inondations (33,8\% de la valeur agrégée), de la recharge de la nappe phréatique $(23,2 \%)$, de la rétention des sédiments $(12,4 \%$ ) du pâturage $(10,4 \%)$, de la récréation $(8,9 \%)$ et des poissons $(7,0 \%)$ en plus du rôle joú par les forêts dans la rétenrion des teres (2,3\%) et la séquestration du carbone (19\%) a production d'olves $(0,2 \%)$, la production du mie $(0,4 \%$, lich et rutilsation des eaux thermalos $(0,4 \%)$. Laugmentation de a frequence des evenenements extrêmes previsibles avec les dans l'atenuar an a dans l'atténuation des effets du changement climatique.

La valeur des services écosystémiques obtenue (498 DT/ha ou 254 \$/ha en 2015) est supérieure à la moyenne mondiale (74 \$/ha - prix 2015) ou celle estimée récemment pour les orêts tunisiennes (176 DT/ha en 2012). En effet, le PNI n'est pas un écosystème comme les autres en termes paysagers, ecologiques, et économiques, grâce à la coexistence et les interactions dynamiques entre les trois sous-écosystèmes. C'est le lac qui dégage le plus de bénéfice (526 DT/ha), grâce notamment aux multiples services de régulation. C'est la population des villes et villages voisins qui profite de la plus grande partie de la valeur economique des services fournis par le parc à travers les services relatifs à la régulation de l'eau, la récréation, etc. Malgré la faible part profitant à la population locale (11\%), la valeur du bénéfice profitant à la population locale est assez élevée : soit 3089 DT par ménage par an pour la population à l'intérieur du parc et 2043 DT ménage par an pour la population à l'extérieur du parc.

La valeur des services écosystémiques excède le coût gestion et protection du parc, donc, les dépenses consenties pour la conservation du parc (656.000 DT/an) sont justifiées économiquement. Plus spécifiquement, les valeurs attribuées à certains services écosystémiques (récréation, poissons) sont supérieures aux coûts consentis pour leur maintien.

Comme les visiteurs ont montré une disposition à payer un droit d'entree au parc de 2,5 DT en moyenne pour les adultes et de 1 DT pour les enfants, cela signifie que l'écotourisme pour rait constituer une source de financement intéressante du parc.

\section{Liens entre l'état de}

l'écosystème et l'importance des services écosystémiques

est communément admis que la conservation de la biodversite constitue un support pour la fourniture des services ecosystémiques. Dans le cas du PNI, plusieurs auteurs on souligné les effets négatiff de la baisse du niveau d'eau du lac et de sa salinité sur la biodiversité et ses impacts sur les services écosystémiques. Toutefois il n'y a pas eu de fortes baisses des flux des services (poissons, écotourisme, pâturage, etc) observés pendant la période 2000-2015 malué évènements de sèchese II est probable que la resiSence de lecosysteme les eftors de preservaton du parc ence de lecosysteme et les efforts de pre nt joué un rôle positif dans ce sens.

y a donc un besoin de développer la recherche pour l'établissement de modèles intégrant a la fois les aspects écologiques et économiques. Ces modèles devront être app qués pour les pratiques de gestion existantes, ainsi que pour les scénarios de gestion étudiés
Valeur de la conservation des habitats et de la biodiversité

Le bénéfice lié à l'amélioration de la biodiversité est estimé à partir de la disposition à payer des populations concernées, a savoir : les usagers du parc (population locale à lintérieur et à l'extérieur du parc), les visiteurs, ainsi que le public en général. La valeur globale de la disposition à payer par a

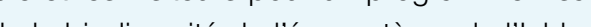
est estimée à 1,3 Million DT, soit l'équivalent de 104 DT/ha en moyenne.

'enquête effectuée auprès des communautés locales sur disposition à payer pour l'amélioration de la biodiversité de l'écosystème Ichkeul montre une attitude très positive, toulefois, cette attitude est à base utilitaire, relative à l'amélioration des services écosystémiques (paturage, services lies lu hamm, etc). L'enquete aupis des visteurs presente

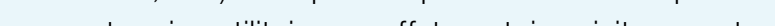
un aspect moins uthlaire, en effet, certains visteurs ont exdiversité, et non pas pour tirer profit de ses services. L'enquête auprès du public, aussi bien national qu 'internationa, a également montre une forte disposition à payer, considérée principalement comme une valeur de non usage. La démonstration des valeurs d'existence au niveau international permet de justifier les prêts et donations internationales pour la protection du parc.

La prise de conscience pour lamélioration de lenvironnement et du cadre de vie dans le futur contribuerait à un meilleure perception de l'amélioration de la biodiversité par - part plus large de la population.

La valeur attribuée à la conservation de la biodiversité pourrait justifier des lâchers d'eau dans le lac (de 16 à 44 MilIon $\mathrm{m}^{3}$ tous les 3 ans selon l'usage alternatif de l'eau). Cette quantite correspond au même ordre de grandeur que la demande environnementale de l'eau (20 Million $\mathrm{m}^{3}$ ) indiquée par l'ANPE en 2003. 


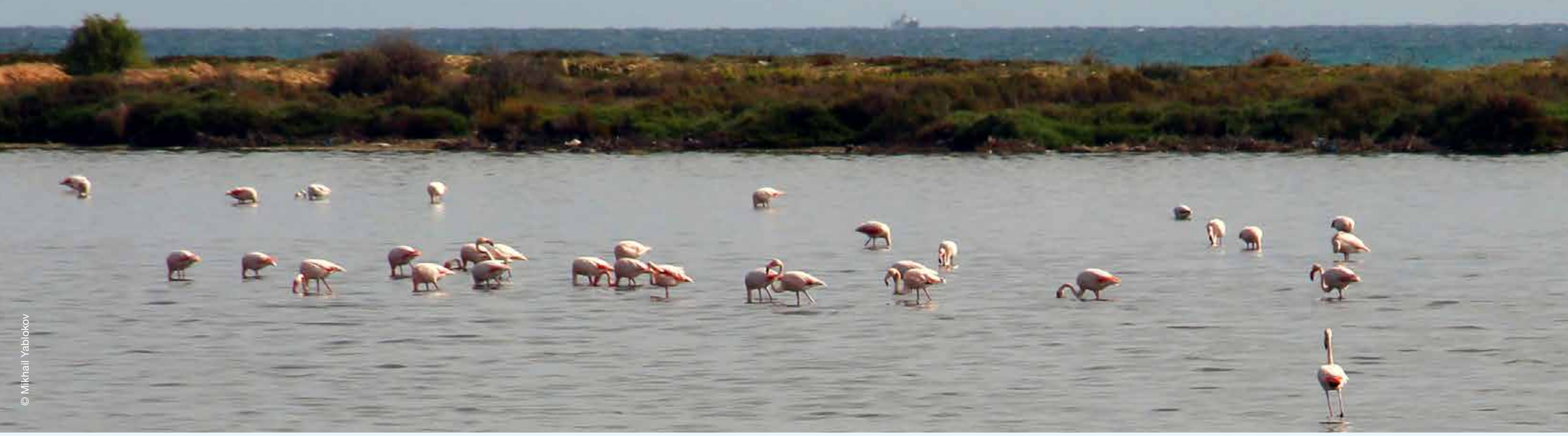

Recommandations

La compréhension de l'ensemble des valeurs de la conservation de la biodiversité et des services fournis par le Parc National Ichkeul (PNI) nous amène à aider à la prise de décision dans la gestion du parc :

1 Renforcer la contribution du lac dans la protection des terres agricoles en amont de la zone humide et de la ville de Mateur contre les inondations afin d'accroitre la valeur des bénéfices du parc. Actuellement, une superficie de 1000 ha environ est encore soumise a des risques d'inondation. D'autant plus, ce rôle bénéfique pour la population de la région est accompagné par une amélioration de léquilibre de l'écosystème lacustre (situation gagnant-gagnat).

2 Considérer les bénéfices multiples du PNI dans la gestion de l'écluse, et ne pas se focaliser simplement sur les conditions favorables pour développer un service écosystémique particulier. Plusieurs services rendus par le parc ne sont pas reconnus alors que leurs valeurs économiques sont élevées, la protection contre les crues et les inondations, et la recharge de la nappe notamment.
3 Renforcer la participation des résidents du parc, des riverains, des agriculteurs de la région, notamment l'OTD, es responsables de la commune de Mateur et des services liés à la gestion des barrages, ainsi que les autorv́s réviona a ges lon cos butorcomposantes et que la mulfonctonnalie du PNtes les composes soient consideres comme un mocal voire régional. Lamelioration de la gestion doit permettre à la fois de developper certains services comme la récréation, l'éducation environnementale, la baignade, la relaxation et le soin (grâce au hammam) et de faire mieux profiter les différentes parties prenantes des services $d u$ parc sans porter atteinte à l'équilibre de l'écosystème.

4 Rechercher des compromis entre les différents acteurs sur les différents usages (pâturage, pêche, récréation contrôle des inondations, dévasement des barrages), ̀̀ travers la logique gagnant-gagnant, le développement local et la cogestion des ressources naturelles. Ces solocal et la cogestion des ressources naturelles. Ces so-
lutions de compromis vont non seulement assurer une coordination entre les differents bénéficiaires sur la gestion du parc, mais aussi assurer un meilleur partage des coûts et des bénéfices entre le gestionnaire du parc et les bénéficiaires.
5 Mettre en place des mécanismes et dispositifs pour assurer les paiements de certains services écosystémiques par les béneficiares, ou au moins prendre en charge coûts des activités (visiteurs, agriculteurs, etc). L'écotourisme pourrat devenir uns importante source de financement du PNI.
mevenir une importante source de finance-

6 Orienter l'approche de conservation du PNI vers des arguments économiques basés sur l'ensemble des valeurs des services écosystémiques fournies, en plus de ceux sur la préservation la biodiversité. La conception des programmes et des projets de protection du PNI nécessite une nouvelle vision en rapport avec le développement cal l'́ versants. La promotion du lac comme une destination versants. La promotion du l'ce comme une destination touristique et como site deducation environnementale pourrait aider à accroitre la sensibilisation sur la valeur du PNI et limportance de son utilisation rationnelle. Ceci permet aussi de creer des revenus au niveau local et de drainer des fonds nationaux et internationaux. A l'échelle mondiale, l'écotourisme progresse de 20\% par an.
7 Utiliser les valeurs des services écosystémiques comme moyen de communication sur le rôle du parc dans l'économie locale, régionale et nationale, afin d'obtenir l'adhesion pour la gestion du parc, l'attraction de financements pour des mesures de protection et de gestion, et réduire les pressions sur le parc.

\section{Perspectives}

llest nécessaire de développer des connaissances scientifiques afin de minimiser les coûts de dégradation. Plusieurs questions restent posées : Quels sont les effets de certains usages, tels que le surpâturage, le dévasement des barages sur la conservation de la biodiversité et la durabilité des services écosystémiques? Ouls sont les effets à long des sorvices écosystemiques? Quels sont les effets a long 作 thes sur les fiux des services ecosystemiques ? Aussi, les miqnifiques devraient développer des instruments econoun meilleur partage des coûts et bénéfices entre les parties prenantes, et de faire impliquer de façon coordonnée les différents acteurs dans la gestion du parc. 
Les zones humides occupent une grande partie de la surface de la Terre. Elles fournissent des services écosystémiques vitaux tels que la régulation des crues, l'épuration des eaux la fourniture d'eau, la pêche, les activités récréatives. Elles jouent un rôle important dans l'atténuation et l'adaptation au changement climatique, et soutiennent le developpement economique (par exemple hyydroelectricie). Les zones riches en biodiversité foumissent des opportunités pour la recherche scientifique, les loisirs et l'éducation. La valeur économique totale des benefices de quelques 63 millions d'hectares de zones humides dans le monde est estimée à environ \$3,4 milliards par an, soit \$54/ha (Schuyt et Brander, 2004).

II y a plusieurs bénéficiaires des écosystèmes à léchelle locale, nationale et internationale qui ont des perceptions divergentes concernant leurs utilisations. Cette multitude $d$ services des zones humides et de leur dimension spatiale souligne le compromis essentiel entre les services de régulation des zones humides et les les services d'approvisionnement pour les utilisateurs locaux.

La question de l'évaluation de la biodiversité considéré comme suppr a été tratée par plusieurs auteurs depus 1977 : Westo (1977), Randall (1988), Pearce et Moran (1994) et Perring (1995) notamment. Cette question provoque un large débat, tant au sein de la communauté scientifique, qu'auprès des décideurs publics susceptibles d'en utiliser les résultats. La volonté de développer une vision commune s'est traduit par trois grandes initiatives internationales : le Millennium Ecosystem Assessment (MEA, 2005) et The Economics of Ecosystems and Biodiversity (TEEB, 2010), et récemment, P'IPBES (Intergovernmental Science-Policy Platform on diversity and Ecosystem Services, Nations Unies).
Lévaluation de la valeur économique des services écoun certain ordre de tes teclaringes necessares a la prise de detes éclairages nécessaires à la prise de de-

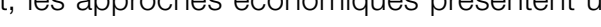
con pres des choix dans cosnurvation es putilisation durable des differentes composantes de l'ecosystème. Lanalyse economique de l'ecosysteme se donne non seulement pour objectif d'apprecier es benefices, mais aussi de les comparer par rapport aux coûts des programmes de préservation de l'écosystème qu peuvent genérer plus de bénéfices que de coutt Durant les années de sècheresse, il y a une demande de la part des or ganisations de protection de l'environnement pour que le lac chkeul sol alineté par les eaux du barrage afin de mainteen biodiversité (oiseaux migrateurs), alors que cette ressouce rare est générement allún à latis que tion en eau potable et à l'irrigation.

Une telle approche est de nature à contribuer à l'amélioration de l'état de conservation des aires protégées et des zones clés pour la biodiversité et à mieux les intégrer dans leur contexte social et économique. Ceci permettra ainsi de concier les objectifs du PNI avec les attentes et les aspirations des bénéficiaires. Montrer comment le Parc National contribue, ou peut contribuer, dans l'économie de sa région, voire du pays, enforcera sa conservation à travers une meilleure acceptation et une meilleure vision de son intérêt.

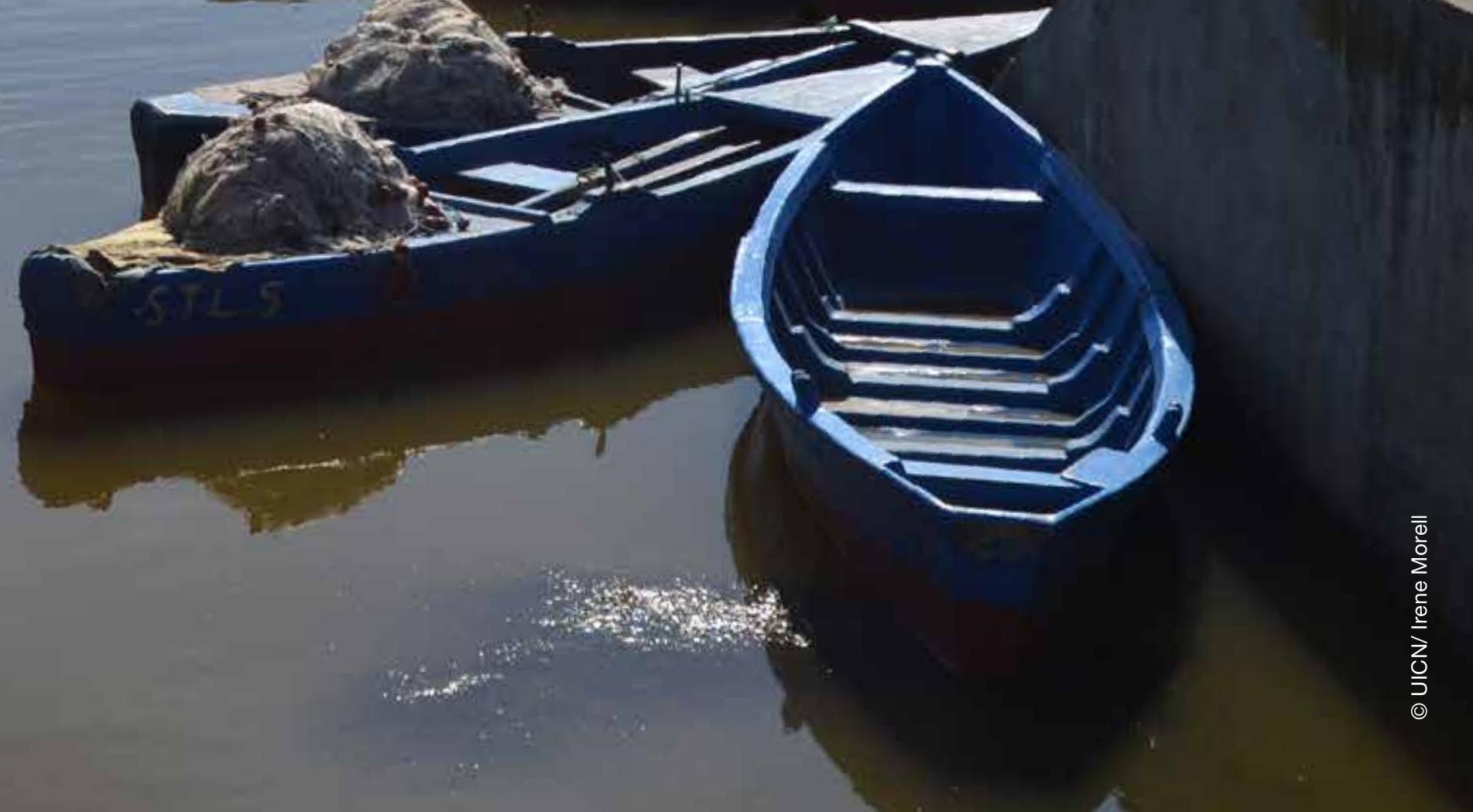


1.1 Présentation du Parc National de l'Ichkeul

Le parc Ichkeul se situe au Nord-Est de la Tunisie à 75 Km de (1363 ha), le lac Ichkeul (8500 ha), et les marécages (2737 ha) Tunis, à $25 \mathrm{~km}$ au Sud-ouest de Bizerte et à $15 \mathrm{~km}$ des villes (cf. Carte 1). Le parc est accessible via la route nationale goude Mateur et de Menzel Bourguiba. II se situe sur la plaine de dronnée GP 11 reliant les villes de Menzel Bourguiba, Tinja et Mateur entre les chaînes de montagnes de Mogods et la mer. Mateur. Il est bordé du côté Nord, Sud et Ouest par des terres Ml occupe 12.600 a courant successivement, la montagne a aricoles privées et du coté Est par la ville de Tinja.

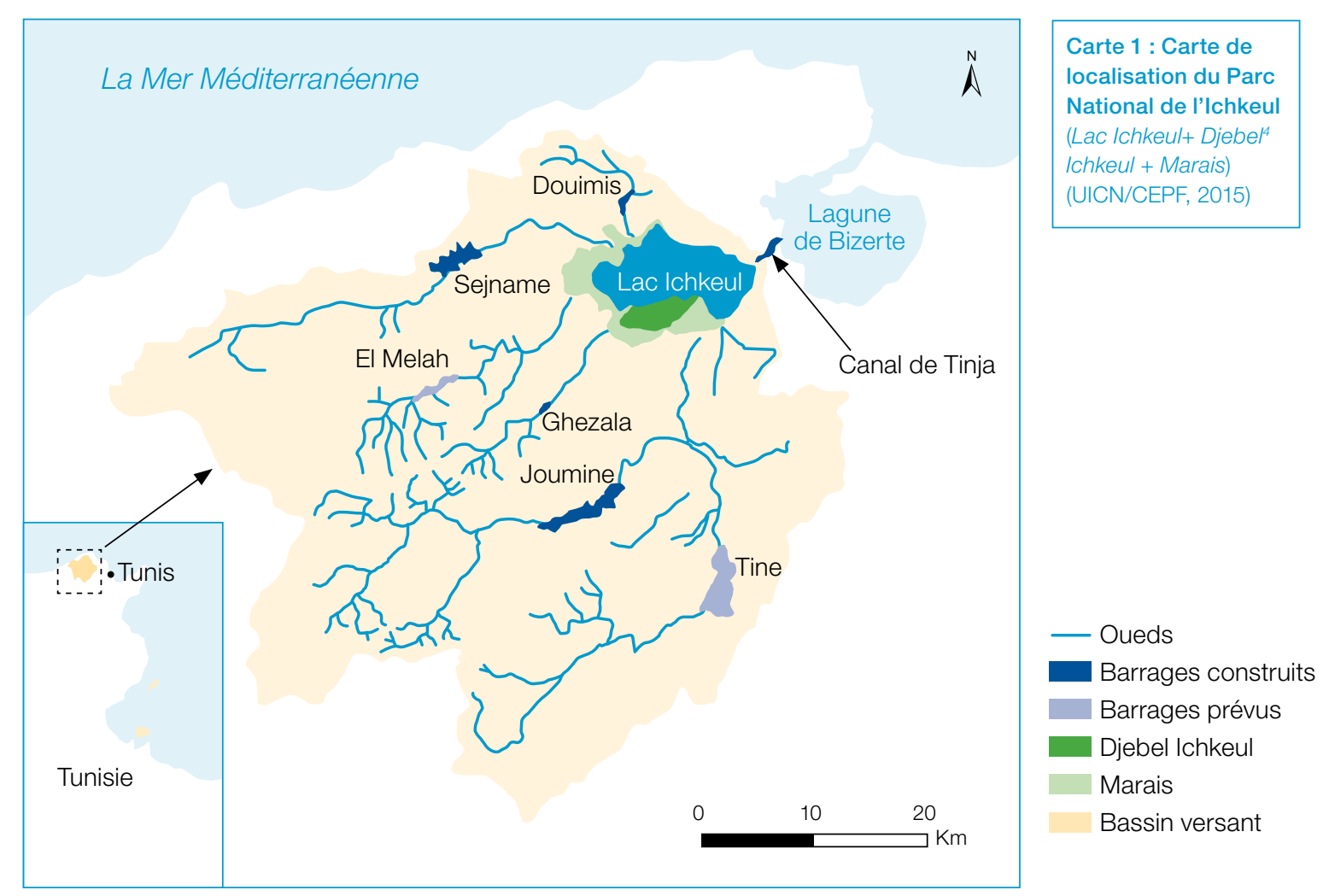

IMPORTANCE INTERNATIONALE : La zone humide de Ces conventions est donc la conséquence logique d'un l'Ichkeul représente un écosystème très varié en Tunisie. Elle prise de conscience environnementale à léchelle nationa est 'une des quatre principales zones humides du bassin mé- et internationale, traduite en grands engagements lors de diterraneèn avec la Camargue en France, Donana en Espagne conferences reunnissant de nombreux pays, eux-mèmes et El Kala en Algérie. Son importance écologique et interna- adaptés aux enjeux nationaux de préservation de la bioditionale pour l'hivernage des oiseaux d'eau du paléarctique versité (Saied et Elloumi, 2007). Le Parc National de l'chkeu occidental justifie son inscription au titre de trois conventions est l'un des plus importants sites d'hivernage d'Afrique du internationales. Le Parc est classé «Réserve de la Biosphère» Nord pour les oiseaux d'eau du paléarctique occidental. Son par l'Unesco depuis 1977 à travers le programme MAB (Man système laguno-lacustre favorise le développement d'une

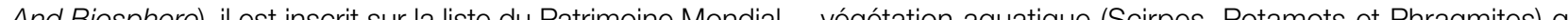
naturel de l'Unesco depuis 1979 et il est inscrit dans la liste sert de support nutritif aux oiseaux migrateurs et de lieu de en dimportance internationale comme habi- développement pour les poissons du lac. ats de la Convention de Ramsar depuis 1980

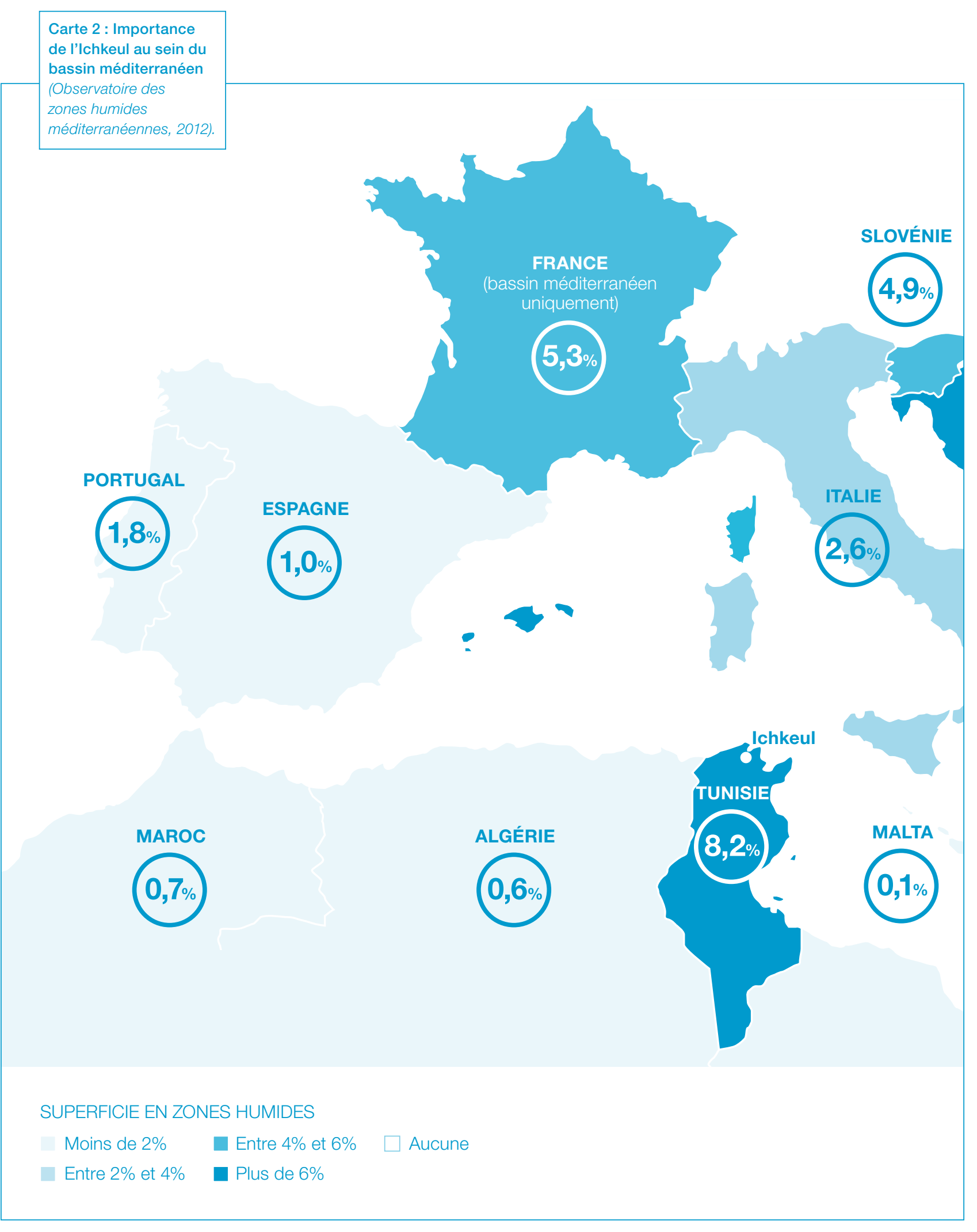

Djebel (mot arabe) signifie montagne 
HISTOIRE : Au XIII’̀me siècle, sous la dynastie hafside, le GÉOLOGIE ET GÉOMORPHOLOGIE : La région de l'Ichkeul site a été institué réserve de chasse. Ce n'est qu'à partir constitue la bordure orientale du domaine tellien maghrébin. 1948 que le statut foncier de cet espace a été officialisé Les ensembles morpho-structuraux qui la composent jouent

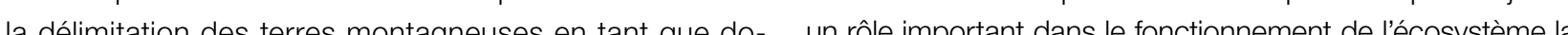
maine public, lo lac a cté institué Domes en tant que do- un

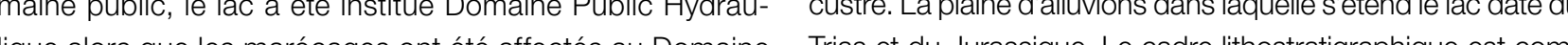
Public et qu Tes marecages ont ete affectés au Domaine Thas et du Jurassique. Le cadre lithostratigraphique est comPublic et aux Terres Domaniales. Lintérêt international de posé de plusieurs massiffs et complexes appartenant au Trias, ce site a été officialisé pour la première fois en 1965 par la au Jurassique, au Crétacé, à l'Eocène, à l'Oligocène, au Miodéclaration du site comme zone humide d'intérêt interna- cène et au Pliocène. Le Djebel, constitue de roches calcaires tional (UICN). En 1974, l'Etat tunisien a confié à la Direction dolomitiques et de marbre, datant du Trias et du Jurassique. Générale des Forêts (DGF), relevant du Ministère de l'Agriculture, la gestion des marais.

A partir de 1976, les marais ont été clôturés, et la chasse la pêche ont ete interdites dans len e plan d'eau. Les operations de classement se sont intensifiées à partir de 1977, suite a son inscription au titre des conventions internationales et sa désignation en tant que Parc National en 1980

CLIMAT : Le PNI est classé dans l'étage bioclimatique méditerranéen subhumide à hiver doux.

- Pluviométrie : La moyenne annuelle de la pluviométrie est de l'ordre de $625 \mathrm{~mm} / \mathrm{an}$ avec des fluctuations assez importantes selon les années (de $360 \mathrm{~mm}$ à $860 \mathrm{~mm}$ ).

LePNI (et sa perripherie) se caractérise par deux saisons bien tranchées : (1) Une saison humide, d'octobre à fevvrier où les moyennes pluviométriques mensuelles peuvent atteindre une centaine de $\mathrm{mm}$ (décembre et janvier) ; (2) une saison sèche, de mars à septembre, avec des moyennes pluviométriques mensuelles nettement plus basses (2 mm en juillet). Les précipitations ont une grande influence sur la salinité de leau du lac qui peut varier, selon les années, de 1,5g/l en hiver à $70 \mathrm{~g} / \mathrm{l}$ en été.

Température : La température moyenne annuelle est de l'ordre de $18^{\circ} \mathrm{C}$ avec un minimum en janvier- février $\left(11,7^{\circ} \mathrm{C}\right.$ et un maximum en août $\left(26,3^{\circ} \mathrm{C}\right)$. Les mois les plus froids sont les mois de décembre et janvier : les mois les plus chauds, juillet, août et septembre.

- Vents : En éte, les vents sont chauds et secs. Durant les mois d'hiver, des vents dominants de NW se manifestent sur toute l'étendue du Lac entrainant l'apparition de vagues impressionnantes. La vitesse moyenne annuelle est de $4,45 \mathrm{~m} / \mathrm{sec}(7 \mathrm{~m} / \mathrm{s}$ en hiver et $2,5 \mathrm{~m} / \mathrm{s}$ en éte).

- L'évaporation : Le plan d'eau du lac est soumis à une forte mum est enregistré en juillet (201 mm) et le minimum en $\mathrm{h}$ ver $(63,3 \mathrm{~mm}$ en janvier). L'évaporation entraine une hausse considérable de la salinité durant la saison estivale.
En outre le lac de superficie variant de $78 \mathrm{~km}^{2}$ à $110 \mathrm{~km}^{2}$ Un modèle mathématique, tenant compte notamment de en hiver reçoit une moyenne de $650 \mathrm{~mm} / \mathrm{an}$, ce qui génère la salinité, gère normalement le fonctionnement de l'écluse n apport direct moyen de $63 \mathrm{Mm}^{3}$. '’évaporation moyenne l'objectif de cet aménagement est de maintenir un équiannuelle est estimée à $122 \mathrm{Mm}^{3}$. Le lac Ichkeul étant relié à libre écologique qui conditionne la vie des zoocénoses et lagune de Bizerte par l'Oued Tinja. En période estivale le des phytocénoses de cet écosystème lacustre. Ce milieu lac Ichkeul reçoit de l'eau de la lagune (environ $64 \mathrm{Mm} 3$ ) et nécessite une gestion rigoureuse, notamment suite (i) aux en hiver, c'est plutôt le lac qui se déverse vers la lagune (345 aménagements hydrauliques (barrages) réalisés au niveau $\mathrm{Mm}^{3}$ ). Les infiltration des eaux du lac permettent d'alimenter du bassin versant du lac de l'Ichkeul et (ii) aux années de la nappe de Mateur d'un volume moyen de $15 \mathrm{Mm}^{3} /$ an. Le sécheresse qui apparaissent souvent de façon imprévisible. ac reçoit également les eaux de drainage de la plaine de Mateur. Le débit annuel varie entre 1 et $4 \mathrm{Mm}^{3} /$ an

CADRE INSTITUTIONNEL, JURIDIQUE ET FONCIER: Les Parcs Nationaux font partie du domaine de l'Etat. Placés Les apports à l'Ichkeul sont caractérisés par une très grande sous la tutelle de la Direction Générale des Forêts (DGF), ils variabilité annuelle et saisonnière. En effet, pour une période sont créés par Décret Gouvernemental en application des $150 \mathrm{Mm}^{3}$ alors qu'ills sont de $650 \mathrm{Mm}^{3}$ en année très humide. été immatriculée en 1948 sous les titres fonciers $n^{\circ} 145$-628 Cette particularité des écosystèmes méditerranéens a été ; ;le lac est intégre au Domaine Hydraulique et les marécages bien prise en compte lors de la réalisation du modèle hydro- en partie au Domaine Public et en partie aux Terres Domalogique de prévision élaboré en 1996. En effet, la série histo- niales. Les marais orientaux ont été cédés en 1974 à la DGF. rique, période de référence du modèle, est de 1953 à 1992 a été caractérisée par le maintien des différents écosystèmes en dépit de successions d'années plus ou moins sèches. a salinité du lac constitue un paramètre fondamental pour l'écosystème. Durant un cycle annuel les eaux du lac sont

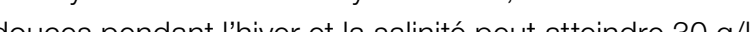

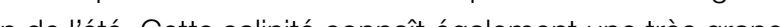

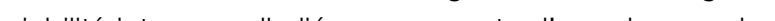
variabilité interannuelle liee aux apports d'eau des oueds et lechange avec la lagune de Bizerte. Durant des periodes de sècheresse, la salinité peut dépasser $60 \mathrm{~g} /$. 
1.2 Objectifs de l'étude

La zone humide est soumise à des risques de déséquilibre de l'écosystème liés à la réduction des ressources en eau au niveau du lac et sa salinité en cas de sécheresse ; les lâchers eau des réservoirs permettent de prevenir la dégradation de l'écosystème. Le pâturage excessif dans les marais, notamment, induit un conflit entre la population locale et les gestionnaires concernant la dégradation de la végétation des marais du parc. L'équilibre de l'écosystème et la préservation de sa biodiversité (espèces d'oiseaux et plantes) dépendent de la gestion du parc et de l'alimentation en eau douce (pluviométrie, lâchers des barrages). Cet équilibre est nécessaire pour assurer une production durable des services écosystémiques.

Lévaluation économique des bénéfices de l'écosystème Ichke peut apporter des réponses par rapport à ces deux enjeux

1 Quels sont les impacts de la gestion actuelle sur la distribution de la valeur des services écosystémiques pour les différents bénéficiaires ?

2 Les lâchers d'eau sont-elles justifiées économiquement (arbitrage conservation / développement économique)?

Les objectifs de cette étude sont les suivants

1 Développer une évaluation de la valeur économique des services écos "lchkeul (PNII). Cette évaluation permettra de renforcer les connaissances sur le site et de sensibiliser davantage les décideurs et les usagers pour assurer une conservalion et une gestion durable de son patrimoine naturel et culturel. L'évaluation apportera aussi des éclairages né cessaires à la prise de décision afin d'assurer la conservation et l'utilisation durable des différentes composantes de la biodiversité. Ceci permettra aussi de concilier les objectifs du PNI avec les attentes et les aspirations de la population locale.
2 Comparer les bénéfices fournis par le PNI aux coûts des programmes de sa réhabilitation / protection (ou conservation). Durant les années de sécheresse, il y a un besein d'alimenter le lac Ichkeul par les eaux des barrages afin

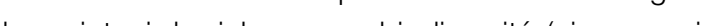
de allouée à l'alimentation en eau potable et à l'irrigation.

Afin de répondre à ces objectifs, les activités suivantes ont te menees :

- Etablissent d'une liste des services écosystémiques fournis au niveau du PNI et leurs caractéristiques, ainsi que leur perception par la population locale

- Evaluation de la valeur économique agrégée des services écosystémiques fournis par le PNI

- Estimation de la contribution de la biodiversité et de l'écosystème en général aux bénéfices des populations locales du Parc National de l'Ichkeul

- Développement des recommandations pour la consolidation de cette initiative et la valorisation de ses résultats (utlisations des valeurs dans l'analyse économique, la prise de décisions, etc.). 
2.1 Méthodologie d'analyse

Plusieurs approches ont été développées afin d'évaluer la valeur des services écosystémiques : on cite notamment TEEB, 2010 et Daly-Hassen, 2013. Plus récemment, l'IPBES a élaboré un guide méthodologique sur les concepts des valeurs multiples de la nature et de ses bénéfices. ${ }^{5}$ Le Groupe d'évaluation scientifique et technique de la Convention de Ramsar a pris note des directives de l'IPBES et prépare un cadre méthodologique pour améliorer l'évaluation des diverses valeurs afin de soutenir la gestion intégrée des zones humides (Information fournie par Dr. Ritesh Kumar, Wetlands International South Asia, Inde).

L'approche d'analyse comprendra les étapes suivantes:

1 Identification et classification des services écosystémiques : II s'agit de lister les biens et services fournis par e PNI selon la classification internationale commune des services écosystémiques (CICES). Une consultation des références existantes a permis d'examiner les informations disponibles.

2 Analyse qualitative et quantification biophysique des services ecosystemiques selon lapproche TEEB. II s'agit d'établir le lien entre l'état de l'écosystème et l'importance des services fournis (usages, activités pratiquées) d'une part, et de déterminer l'état des paramètres environnementaux (eau, végétation) et de leurs impacts sur l'équilibre de l'écosystème d'autre part.

3 Une définition de méthodes d'évaluation pour les difféents biens et services non marchands. Selon le service, ces méthodes peuvent être des méthodes basées sur le prix du marché, le prix des produits de substitution les coûts de dommages évités, la méthode de coût de transport, ou aussi la méthode d'évaluation contingene. Cette étape s'inspire aussi des méthodes appliquées zones humides.

2.2 Classification des biens et services des écosystèmes

4 Collecte de données (cf. 2.6) en vue de l'application des methodes identifiées pour l'évaluation de l'ensemble des biens et services.

5 Conduite des enquêtes auprès de la population locale et des visiteurs du parc pour collecter les données selon 1 méthode d'évaluation adoptée.

6 Estimation de la valeur des différents services, ainsi que Estre les bénéficiaires.

7 Comparaison de la valeur des biens et services obtenue avec celles d'autres zones humides dans le monde.

8 Estimation de la valeur économique de la conservation des habitats et de la biodiversité.

Comparaison des bénéfices rendus par rapport aux coûts des programmes de préservation du lac. Cette comparaison sera effectuée dans deux scénarios :

a. Absence d'eau supplémentaire alimentant le lac.

b. Versement d'une quantité d'eau additionnelle pour assurer l'équilibre de l'écosystème en année sèche. La valeur economique de l'eau sera basée soit sur performance économique des périmètres irrigués solt sur la valeur de pompage de l'eau du barrage pouvant alimenter le lac.
Les écosystèmes procurent de nombreux biens et services contribuant au bien être humain. Les services résultent des conctions écologiques qui semblent être en mesure de souenir ou protéger les activit́s humaines de production ou consommation, ou d'affecter le bien-être en général (Dic-

Définitions des termes relatifs à l'évaluation économique des services de l'écosystème

SERVICES ÉCOSYSTÉMIQUES : Bénéfices résul- VALEURS : La valeur reflète les besoins et les pertant des fonctions de l'écosystème (par exemple, ceptions des individus pour améliorer leur bienune meilleure pêche et chasse, une eau plus propre, être. Définie en termes strictement économiques, la une meilleure vue, une réduction des risques sur la gamme complète des valeurs des services écosyssanté humaine et des risques écologiques). Ceux-ci témiques comprend la volonté de payer de chaque exigent une certaine interaction avec les humains, personne en unités monétaires pour chaque service ou tout au moins une certaine appréciation. Toute- agrégée pour toutes les personnes et tous les serfois, ils peuvent être mesurés en termes physiques vices des écosystèmes. L'ensemble de la volontét

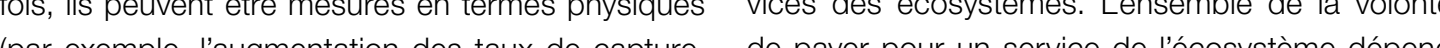
(par exemple, laugmentation dos taux de capture, de payor pour un service de libcosysteme dépend

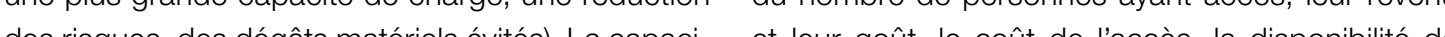
des risques, des dégats materils evites). La capaci- et leur goun, le cout de lacces, la disponibilité de té d'un écosystème à fournir des services peut être substituts, et d'autres facteurs lies à l'offre et la de estimée sans jugements ethiques ou subjectifs sur mande locale, régionale et nationale (King, Wainger, la valeur des services. Les types de services pos- 2001). La capacité d'une zone humide à produire sibles dépendent dans une certaine mesure des un service est à relier avec l'opportunité (sociale et fonctions assurées par l'écosystème, mais surtout économique) de ce service, pour fonder une valeur sur d'autres facteurs (par exemple, l'accès, la proxi- （National Research Council, 2005cité par Agence de mité de la population ou des dégradations causés l'eau Adour-Garonne, 2009.

par la population) (King, Wainger, 2001). En bref, $\mathrm{ce}$ sont les bénéfices que retirent les individus à partir de l'écosystème donné (MEA 2005).
Un bien ou un service a de la valeur, en terme économique, à ravers l'amélioration du bien être humain. Pour cela, l'étude The Economics of Ecosystems and Biodiversity " (TEEB 2010), supporte l'idée que la vision économique de la biodi-

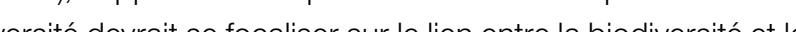
bien être humain. Son objectif étant d'utiliser les approches informations. Son objectif etant duttiliser les approches lees à la degradation de la biodiversite) afin de develop- er des instruments politiques : (dés-incitations)/incitations Payement des services environnementaux, taxes, compensation), et règlementations (normes, aires protégées, investissement public). Des valeurs économiques peuvent être ttribuées aux pertes de services des écosystèmes, même si certains services de soutien peuvent avoir une valeur intrinsèque indépendante de son utilité sociale (conservation des espèces animales et végétales). 
Différents avantages des écosystèmes pour la société.

L'ensemble des services produits par les écosystèmes n peut être évalué économiquement et n’a pas toujours une valeur marchande. Ils ne peuvent donc être intégrés totalement dans la comptabilité nationale. La valeur des services produits par l'écosystème.

La nature des biens et services (biens privés, biens com muns, biens publics et externalités) peut être associée au type de benéficiaire : population à linterieur du parc (beneficlaire de certains droits d'usage), population riveraine (beneficiaire du pâturage), la population nationale, la communauté globale, l'Etat et le secteur privé. Cette classification permet de dégager les divergences ou les complémentarités pouvant apparaitre entre les utilisateurs des biens et services, en vue de développer un meilleur processus de coordination et de gouvernance entre les différents acteurs en vue d'une meilleure durabilité.

Conformément aux objectifs visés par cette évaluation économique, les services écosystémiques sont classifiés selon les catégorisations internationales telles que MEA, CICES (Staub C. et al. 2011). Cette approche permet d'identifier trois and dervices définis en tant qu'avantages procurés aux individus (bien être humain) selon MEA (2005) (cf. Figure 1) :
- Les services d'approvisionnement/prélèvement, ce Les d'approvisionnement/prelèvement, ture, l'eau douce, le bois, les ressources génétiques, etc. IIs permettent notamment de procurer des revenus, l'accès à 'eau, à une source d'énergie et à un habitat sain et propre.

- Les services de régulation, issus de la régulation des processus des écosystèmes (tels que la régulation de l'eau et du climat, la purification de l'eau) qui permettent la réduction des maties, l'accès à un environn prope, l'́volution dans une atmosphe saine.

Les services culturels, ce sont les bénéfices : spirituels, éco-touristique et récréatifs, qui permettent d'établir des bonnes relations sociales, et constituent des opportunités d'extérioriser les valeurs récréatives, culturelles et spirituelles.

La fourniture des services ci-dessus est lié au bon état de lecosysteme. Le fonctionnement de lecosysteme permet le developpement du cycle des elements nutritifis, la constitution du sol et la production primaire, et assure ainsi la conservation des especes et habitats générant ces services. Par exemple, la conservation d'un milieu naturel propice à la reproduction permet de maintenir la pêche, la chasse et cueillette (MEA 2005). A l'inverse, la dégradation de l'écosystème peut entrainer la perte de ces services.

\section{Figure 1 : Les services rendus par les écosystèmes et leurs liens avec le bien être humain (MEA,2005)}

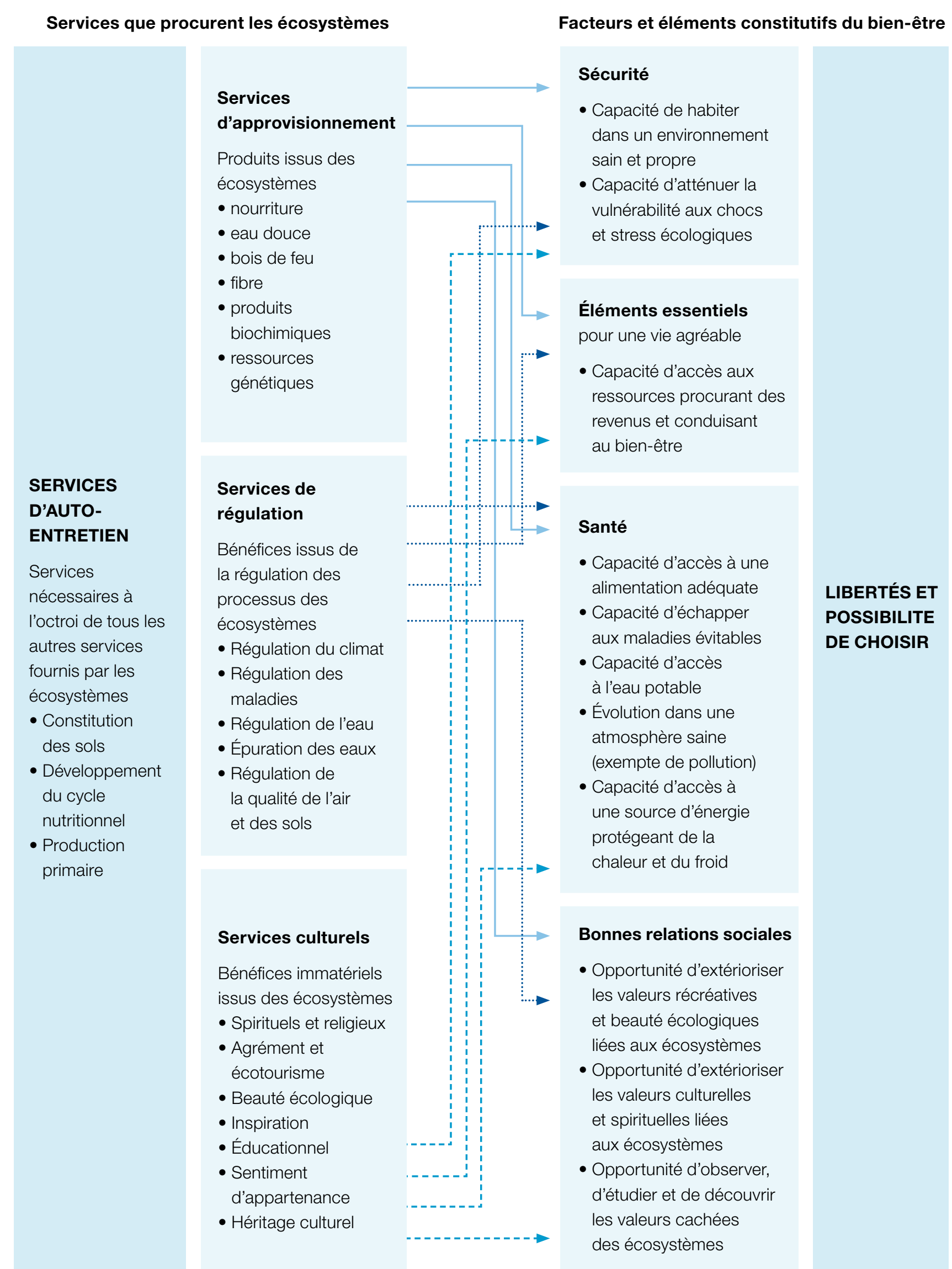


2.3 Concept de la valeur économique totale des écosystèmes

Pour estimer la valeur complète des bénéfices des écosystèmes, le concept de la Valeur Economique Totale (VET) a été développé, il a été souvent utilisé pour estimer la valeur économique des services rendus par les zones humides (Turner et al. 2000). Au-delà des valeurs liées aux bénéfices marchands, ce concepta été dév vé afin de tenir compte les valeurs des bénéfices non machends. II a ́té démonTé que l'une des causes de dégradation et/ou do mavise e que lune des causes de degradation et/ou de maunaise gestion des ressources naturelles est la non prise en consideration de lensemble des bennefices et coûts dans le processus de décision. Leur intégration dans l'analyse rendrat les décisions publiques plus efficaces.

La VET est un concept anthropocentré, qui met l'accent sur la aleur des bénéfices pour les êtres humains. Les composantes de la VET sont ainsi définies de la façon suivante (cf. Figure 2):

- La valeur d'usage direct découle de l'utilisation directe de a ressource. Elle comporte les biens et services destinés des fins de consommation (biens et services présents ou non sur le marché) et à des fins de non consommation (ré-

services d'approvisionnement et aux services culturels, $\mathrm{cl}$ tés plus haut.

La valeur d'usage indirect. Ce sont les biens et services liés aux fonctions écologiques de lécosystème, tels que la régulation des processus hydrologiques.

- La valeur d'option est attribuée par des individus qui désirent pouvoir profiter d'une certaine ressource naturelle - Lavaleur diopion est atribue par des nididus quidelisćs actur. Certans biens et sevices ne sont pas uti-

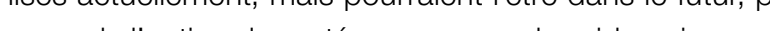
exemple loptron de proteger une zone hus deguipourat avoir du potentiel pour peutiques.

- Les valeurs non liées à l'usage sont attribuées à conservation de certains éléments naturels de la biodiversité (espèces animales et vécétales), les individus sont disposés à payer même s'ils n'en font aucun usage présent ou futur Ces valeus sont attachés à tansission d'un patrimoine à ses descendants (valeur d'héritage/patinopiale) ou à a satistacton de savor quuneressource (valeur d'existence).

Figure 2 : Les composantes de la Valeur économique Totale (V.E.T.) des écosystèmes (M.Merlo and L. Croitrou (Eds), 2005)

\section{VALEUR ECONOMIQUE TOTALE}

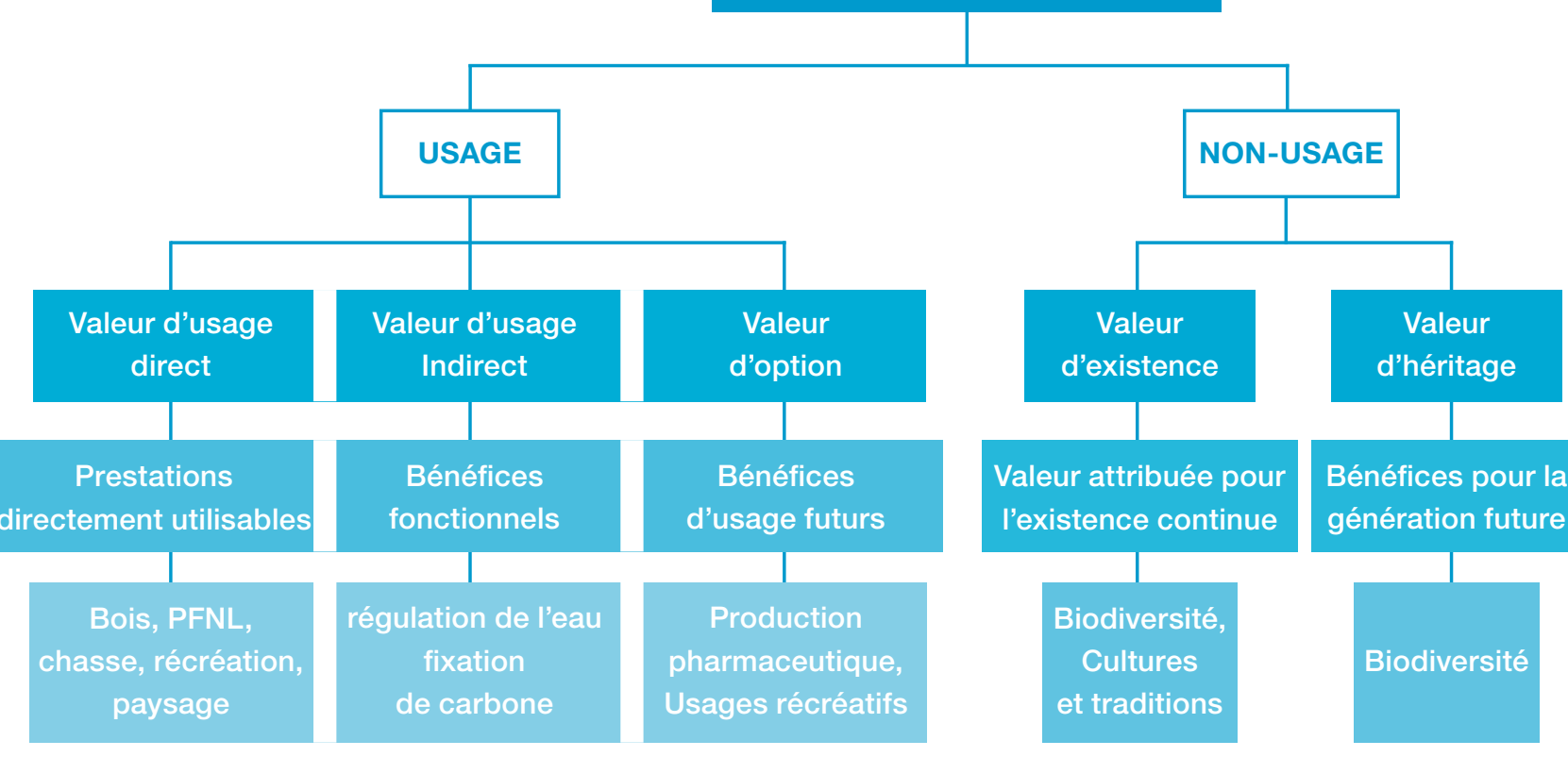

La VET intègre tous les services fournis par l'écosystème, en Par leur nature, les bénéfices d'usage indirect, les bénéplus, elle comprend les valeurs d'option et de non usage cor- fices d'option et de non-usage sont plus intangibles que respondantà des éléments naturels qui ne sont pas utilisées les biens privés, et sont donc, plus difficiles à quantifier. actuellement, mais, pour lesquels les individus sont dispo- Aussi, des précautions sont à prendre pour éviter le double sés à payer. Pour ces biens publics, la valeur économique comptage lors de l'agrégation de l'ensemble des valeurs 'une unité de service correspond à la somme de disposi- pour estimer la VET.

tions à payer de tous les individus pour cette unité (la société

en général), même s'ils n'en font aucun usage.

2.4 Méthodes d'évaluation de la valeur économique des bénéfices

Depuis les années cinquante, la science économique a dé- services. Les méthodes basées sur les coûts consistent à veloppé différentes méthodes pour estimer la valeur des se référer aux coûts pour le maintien ou la restauration des biens et services que les écosystèmes apportent à la so- services écosystémiques. Ces approches exigent d'avoir ciété. On peut utiliser les méthodes d'évaluation fondées sur des informations sur les changements subis par les écosys"les préférences révélées " qui utilisent les dépenses des tèmes en termes physiques et biologiques, ainsi que de leurs consommateurs et les prix des biens et services marchands conséquences en termes d'approvisionnement en services. (cf. Tableau 1). En l'absence de marché et de prix qui fait Lidée de base est que le bénéfice non marchand a une vaapparaître la valeur d'un bien ou d'un service, des méthodes leur au moins égale au coût de son maintien. Les méthodes d'évaluation reposant sur les « préférences révélées » ont été fondées sur les préférences exprimées consistent à obtenir développées, c'est-à-dire les valeurs peuvent être reflétées directement le consentement à payer pour bénéficier d'un indirectement par les dépenses des consommateurs et par actif environnemental en interrogeant directement la popules prix de biens et services marchands. Aussi, les méthodes lation. En effet, les valeurs économiques dépendent de la des «préférences exprimées" évaluent la valeur sur la base perception humaine individuelle des biens et services.

de la disposition à payer des gens pour bénéficier de certain

Tableau 1 : Type de méthodes utilisées dans l'évaluation économique des biens et services des écosystèmes
Méthodes directes

Méthodes indirectes

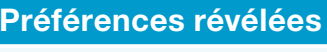

Méthode de marché observés

Méthodes basées sur les coûts : coût de remplacement, dépenses de prévention

Méthode de substitution

de du voyage (MCT)

Méthode des prix hédonistes

\section{Preferences exprimees}

Prix déclarés

d'évaluation contingente (MEC)

- Méthode de choix expérimentaux 
MÉTHODES D'ÉVALUATION DES BIENS ET SERVICES Méthode basée sur le prix du marché : Cette méthode est utilisée pour les produits marchands (olives, graines de lentisque, caroubes).Le prix devrait être local, il ne doit pas inclure ni les coûts d'exploitation, ni les frais de transport ou de marché, ni les coûts de transformation. Les prix de marché peuvent être utilisés pour établir les comptes financiers et dégaer ainsi les valeurs résiduelles des produts locaux. Pour obtenir le prix économique il est parfis nécecaux.

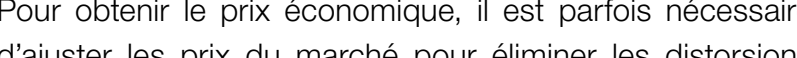
(taxes, subventions, etc.) (Monke and Pearson 1989).

Méthode des prix des produits de substitution : Lorsque es produits sont directement utilisés et ne sont pas commercialisés (fourrage par exemple), une approximation de la valeur peut être effectuée par les prix de marché des produits similaires dans d'autres zones, ou par la valeur des produits resubstitution les plus proches lorsque les produts ont des cubstiuts sur le me turage, il faudrant soustraire le cout de main d'ceuve familiale. Celui-ci est estime à travers le cout d'opportunté du temps qui devrait etre bien ajusté en fonction de refort fourni et les conditions salariales (Bishop, 1999). L'hypothèse est que la decision de passer le temps pour le paturage est equilibre par rapport à d'autres usages alternatiffs du travail familial.

La méthode du coût de voyage : Elle est basée sur le principe que les consommateurs expriment leur consentement à payer pour un site naturel à travers l'ensemble des dépenses qu'ils engagent pour s'y rendre. Ces dépenses intègrent le coût du voyage, le droit d'entree et le coût du temps consacré. Elle est appliquee pour estimer la valeur liee aux activités récréatives. Dans le cas précédant, le bénéfice récréatif peut être déterminé à partir des observations du nombre de dèplacements à but récréatif vers le PNI et du coût de chaque déplacement.

'application de cette méthode nécessite la conduite de six étapes (Brahic et Terreaux, 2009)

-Etape1: Choisir le mode d'enquête.

- Etape2 : Concevoir le questionnaire.

- Etape3 : Estimer les coûts de transport (C)

- Etape4 : Construire la fonction de demande $\mathrm{C}=\mathrm{f}(\mathrm{Nv})$

Nv étant le nombre de visites par an La constuction de ce modèle peut être sous la forme zonale ou individuelle. L'approche zonale consiste à construire des cercles concentriques, de sorte que la distance entre l'aire des deux cercles successifs et le site est identique, et donc ayant un meme cout de transport. Les différences en matière de visite entre les zones, tenan compte des populations, sont expliquées par les différences de coût de transport. Par contre, l'approche in- dividuelle considère les différences de comportement des individus au niveau d'une même zone.

- Etape 5 : Calculer le surplus du consommateur : $V$.

- Etape6 : Calculer la valeur du site : $V \times$ Nombre de visites.

Cette méthode ne prend pas en considération la valeur que peuvent attribuer les non visiteurs au parc, la valeur d'existence.

Méthode de changement de production : Cette ap proche repose sur l'hypothèse que le bien marchand peut epresenter un substitut au bien environnemental. Elle établit un lien entre la production de l'ecosystème (changement mesure de la qualite ou de la quantite) et les benéfices sociaux (accroissement de la production d'un bien ou service marchand), par exemple, cette approche permet d'estimer laugmentation de la production agricole résultant de baisse de l'érosion du sol. La procédure d'évaluation peut tre effectuée en deux étapes :

- Determiner les effets physiques des changements de l'écosystème sur l'activité économique.

- Evaluer les changements résultants dans la production ou la consommation en utilisant des prix de marché (Banque mondiale, 2005).

Cette méthode est appliquée pour apprécier les impacts positifs d'une dégradation évitée en estimant les coûts des dommages évités, par exemple, les impacts d'une plantotion forestière sur la réduction de la sédimentation d'un ac ou un réservoir. Cette méthode est simple lorsque les cosystèmes ont une principale valeur non marchande relative à une seule fonction écologique. Les inconvénients de la onction de production résident dans le fait qu'elle ne permet d'évaluer qu'une partie de la valeur d'usage.

La méthode d'évaluation contingente permet d'évaluer un seul changement (activité récréative, amélioration de la biodiversité, par exemple). Elle consiste à simuler un marché hypoth $\mathrm{p}$ environnementale a defin precisenent Ie mache expermental (qualíté, côth efficacití) puis d'élabore une enquête auprès d'un échantillon repés puis dellabor un enque consentar avantage environnemental.

Lapplication de cette méthode requiert l'application des tapes suivantes (Brahic et Terreaux 2009):

- Etape 1 : Identifier le changement à évaluer.

- Etape 2 : Identifier la population concernée.

- Etape 3 : Déterminer le mode d'enquête et la taille de echantillon

-Etape 4 : Rediger le scénario d'évaluation. Le questionnaire commencerait par décrire l'état actuel de la bio-

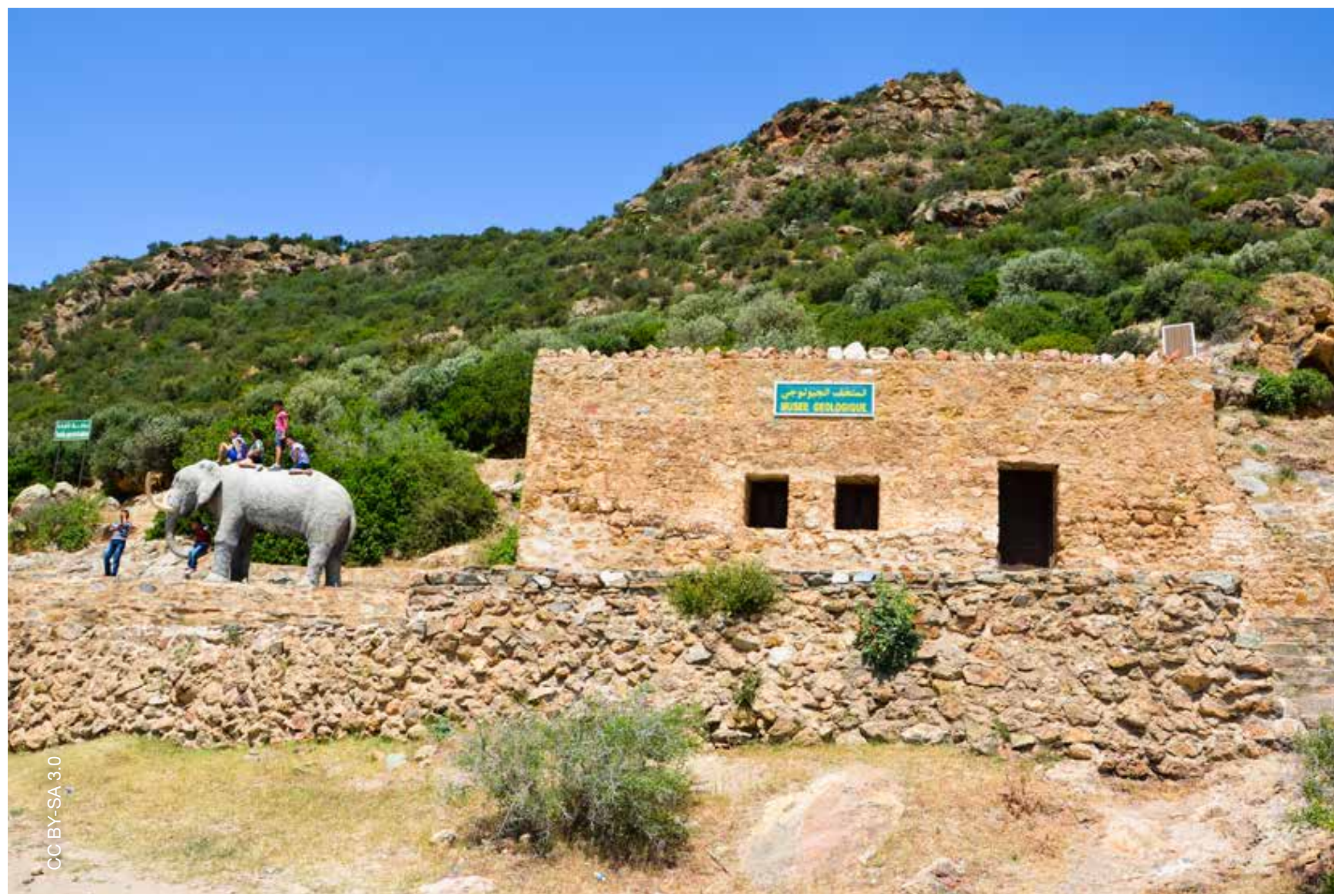

diversité, puis proposerait un changement possible de cette fonction : par exemple, un nouveau plan d'amé- leur d'usage et de non usage) peuvent être déterminées. nagement qui pourrait en améliorer la biodiversité. Mais Les problemes relevent du fait qu'il s'agit d'une situation hyce plan est coûteux et doit être financé. pothétique, avec des intentions de paiement et non pas de -Etape 5 : Rédiger les questions d'évaluation, etc. Des coût subi reellement, que les individus peuvent surestimer questions sont alors posées du type : "Combien se- le consentement à payer pour s'assurer que le service colriez-vous disposes a payer pour la mise en œuvre de ce lectif serait produit (problème du resquilleur) et que les réplan? ».

- Etape 6 : Tester le questionnaire. ponses peuvent être influencées par le type d'informations, le choix de l'instrument de paiement (Bontems et Rotillon, - Etape 7 : Analyser les réponses. A partir de celles-ci, le 2007:Centre d'analyse stratégique, 2009). Cette méthode moyenne du maximum du consentement à payer des budget.

individus de l'échantillon, c'està-dire la valour qu"lls

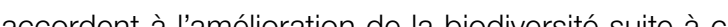
plan. Elle peut etre ainsia depliqué à l'́chele dele a copulation concernée.
La methode du coût de remplacement : Elle donne une valeur au benefice environnemental en estimant son coût de remplacement par le coût des infrastructures nécessaires 
pour fournir un service similaire. Par exemple, l'envasement d'un barrage peut être estimé par le coût de dévasement ou le coût de construction d'un nouveau barrage pour le remplacer. Aussi, lérosion peut être estimée par le coût des fertilisants nécessaires pour remplacer les substances nutritives du sol. Dans ce cas, il faudrait prendre l'option de coût la moins chère pour ne pas surestimer le bénéfice environnemental.

Les problèmes surgissent lorsque le coût potentiel plutôt que les dépenses réelles soient utilisées. L'utilisation de dépenses réelles peut sous-estimer les dégâts, comme les actions remplacent rarement tous les services venant de l'écosystème original. II peut aussi surestimer, car le remplacement peut être entrepris inefficacement. Certains auteurs soutiennent que, dans la plupart des cas, les coûts de remplacement ont tendance à surestimer la valeur des dégâts (Heal, 1999 in Merlo et Croitoru 2005). Les principales faiblesses sont

- La sous-estimation de dommages car certains coûts ne sont pas pris en considération et certains services ne peuvent pas être récompensés.

- La surestimation à travers l'agrégation des coûts d'investissement et des coûts opérationnels. Aussi, les coûts considérés peuvent inclure des dépenses potentielles (non réelles).

La méthode des dépenses de prévention attribue une valeur au service environnemental en estimant les coûts pour prévenir une réduction du niveau de ce bénéfice. Par exemple. es dépenses dans les mesures de conservation des parcs naturels peuvent être utilisées comme une approximation des bénéfices liées à la conservation de la biodiversité

Sur la base des méthodes appropriées pour chaque service et la disponibilité des données, le tableau 2 présente services écosystémiques du Parc National de l'Ichkeul.

\section{pe de bénéfice}

\section{Service d'approvisionnement}

Olives, câpres, caroubes, poissons

Baignade, relaxation et soins (Hammam)

Pâturage

\section{Nectar (production du miel)}

\section{Services de régulation}

Prévention de l'érosion et de la sédimentation

Protection contre les inondations

Recharge des nappes phréatiques

Rétention des sédiments

Régulation du climat : séquestration du carbone

\section{Services culturels}

Tourisme, loisirs, éducation et recherches

Conservation de la biodiversité

\section{Méthode d'évaluation choisie}

Prix du marché

Prix du marche

Prix de substitution

Prix de substitution

Prix du marché

Coût de remplacement

Changement de production (coût des dommages évités)

Changement de production

Coût de remplacement

Coût de remplacement (Réduction des émissions de $\mathrm{CO}_{2}$ par des techniques alternatives)

Méthode des coûts de transport Méthode d'évaluation contingente
2.5 Distribution des bénéfices

En plus de l'évaluation des services écosystémiques, la distribution de leur valeur par bénéficiaire sera effectuée. Cette classification permet d'identifier les conflits ou les complementarités qui peuvent apparaitre entre les utilisateurs des biens et services en vue de développer un meilleur processus de coordination et de gouvernance entre les différents acteurs pour une meilleure durbilité et d' d'un éventur une chengement de gestion sur le bénéfice dis différents acteurs.

Les bénéficiaires concernés sont :

La population locale vivant à lintérieur du parc et qui bénéficie de certains usages directs (bois de feu, fourrages, plantes a usage therapeutique, services lies au Hammam, etc.). La population locale se regroupe en huit groupements communautaires (farch) qui se trouvent au pied du versant Sud de la montagne de l'Ichkeul, soit un total géneral de 398 individus répartis en 104 ménages (DGF 2006). La population résidant dans le parc a beaucoup diminué à la fin du siècle dernier passant de 894 personnes en 1983 à 415 personnes en 1999 (Zaiane, 2004).

\subsection{Collecte des données}

La collecte des données concerne l'ensemble des indicateurs physiques et monétaires dans la mesure du possible, notamment le flux annuel des valeurs pour la periode d'analyse 1996-2015. Les sources de données sont les statistiques officielles et les données fournies par le Conservateur du parc, DG de la Pêche, DGF, ANPE, CRDA Bizerte, OTD, DG BGTH, DG ACTA, SONEDE, les publications scientifiques, BGTH, DGAC

Afin d'appliquer les méthodes indiquées ci-dessus, trois enquêtes ont été effectuées:

- Une enquête sur les produits prélevés au niveau du parc faite auprès de la population résidant à l'intérieur et à l'extérieur du parc. L'échantillon est constitué de 50 ménages à l'intérieur et de 50 ménages à l'extérieur du parc choisis au hasard. Elle a été menée du 9 au 21 décembre 2015. Le questionnaire (cf. Annexe 1) est focalisé sur les usages du parc:

Usages de produits forestiers (bois, olives, câpres, caroubes) : période, fréquence, production, autoconsommation, prix, etc.
- La population locale vivant à proximité du parc, et qui bénéficie du pâturage, services liés au Hammam et poissons. Cette population provient des douars riverains : Felta et Echouk (20 ménages), Taref (30 ménages), Salah bougabrine (20 ménages), Zaarour (50 ménages). Tinja (50 ménages) Bizerte sud (20 ménages), soit au total 190 menages environ (Communication avec H. Ghazouani).

La population nationale bénéficie de valeurs récréatives et educatives, du contrôle des crues et des inondations, de la recharge de la nappe et de la réduction des intrusions marines.

- L'Etat bénéficie de la concession relative à la pêche. Une entreprise privée a l'exclusivité de pratiquer la pêche dans le lac. - Le secteur privé bénéficie de l'activité de pêche.

- La communauté globale bénéficie de la séquestration du carbone, et de la conservation de la biodiversité

-Pâturage : fréquence, période, lieu (forêts, marais). Pêche.

- Activités salariées à l'intérieur et à l'extérieur du parc. Cette enquête permet aussi d'effectuer une estimation des bénéfices marchands pour l'année la plus récente (2015) et une évaluation de l'importance des activités liées à l'usage des ressources (en termes de revenu par ménage).

- Une enquête auprès des visiteurs pour évaluer la valeur récréative (cf. Annexe 3). L'échantillon est de 100 visiteurs environ choisis au hasard. Elle a été menée pendant les vacances scolaires du 15 au 30 décembre 2015.

- Une enquête sur la valeur de l'amélioration de la biodiversité auprès de la population locale, des visiteurs et du public en général. Un questionnaire (cf. Annexe 4) (mis sous forme d'un formulaire google : https : //goo gl/kddm7B) partagé à travers la page du réseau facebook du décembre 2015.

Les trois questionnaires ont eté préparés, puis diffusés auprès des membres du comité de pilotage. 
3.2 Inventaire des principales espèces animales et végétales directement utilisées par la population ocale, les types d'usage et de collecte

II existe plusieurs inventaires : un inventaire détaillé des es- ployé, la divagation des animaux domestiques à l'intérieur pèces animales et végétales a été effectué par le PAG du d'un parc naturel ou d'une reserve naturel, ainsi que leur parc d'Ichkeul (DGF 2006). Aussi, le plan de gestion des visi- survol par aéronefs ». Les usages qui semblent être encore teurs du PNI cite un inventaire exhaustif de la flore établi par permis sont le ramassage du bois mort, le pâturage ayan Fay (1980) (UICN-Med, 2015), dans lequel 500 espèces ont pour objet la nourriture des bestiaux appertenant à l'useger té recensées. Par ailleurs, Ouali et al. (2014) indique que le (domicilí à l'intérieur des forêts) et l'utliliation de certains

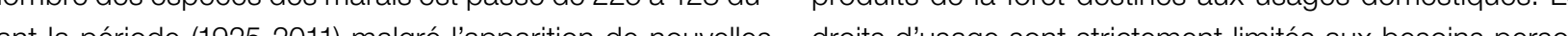

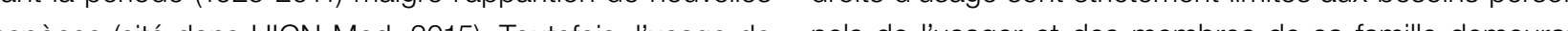
indiqué. avec lui, sans jamais revetir un caracte commercial Aussi, le code forestier précise que la cueillette de produits foresLidentification des espèces utilisées par la population locale le code for ligneux (leurs sauvages, câpres, baies de myrte lenquête et l'étude des prix dans les marchés locaux (Men- etc.) est toleree tant qu'elles ne revetent pas un caracter (Relápublique Tunisienne, 2011). Toutefois, vente de certains fruits est tolérée tant que leur exploitation ne nuit pas à la conservation des ressources. La pêche es pratiquée illégalement notamment pour le loup et le mulet zui sont appréciées par la population de la région.

'laspect genre) et le type du marché pour lequel ce produit Par ailleurs, pour les espèces de poissons, l'étude IUCNlaspect genre) et le type du marche pour lequel ce prodult Par ailleurs, pour les espèces de poissons, létude IUCN est destiné (local, national, vente directe, etc.) sont aus- MED sur " les zones cles pour la biodiversité d'eau douce sí indiqués. Par ailleurs, certaines espèces forestières ont dans le hotspot du bassin méditerranéen " a identifié 9 esete utilisees occasionnellement pour des soins traditionnels peces de déclenchemente dans la zone cle de la biodiversitén (Aouididi 1995) (cf. Annexe 2). Ces espèces n'ont pas été (ZCB): Cap Serrat - Cap Blanc - Parc national de l'Ichkeul (c. mentionnees par les menages interviewes. de la zone, et si 'lusage de ces espèces constitue un facteur (A) dégradation. Pour l'anguille européenne (Anguilla angutSespèce très reconnue à lchkeul les menaces pesant sur

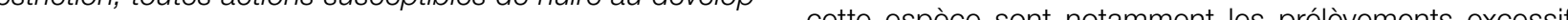
mais en association avec de nombreuses autres activités chasse, la pêche, les activités agricoles, forestières et pas- anthropiques, comme le captage excessif de l'eau, la pollu-

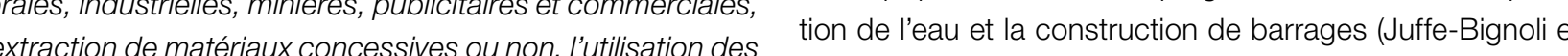
eaux, la circulation du public, quel que soit le moyen em- Darwall, 2012). $\begin{array}{cc}\text { noms } \\ \text { scientifiques } \\ \text { Habitat } & \text { noms locaux }\end{array}$

$$
\text { Habitat }
$$

\begin{tabular}{|c|c|c|c|c|c|c|c|}
\hline & $\begin{array}{c}\text { Oléastre } \\
\text { Olea europea } \\
\text { (زبوس) }\end{array}$ & $\begin{array}{l}\text { familiale des } \\
\text { fruits (graines } \\
+ \text { huile) } \\
\text { Cuisson } \\
\text { du pain }\end{array}$ & manuelle & $\begin{array}{l}14,2 \text { t/an } \\
\text { en } 2015\end{array}$ & $H, F, J^{*}$ & $\begin{array}{l}\text { Consommation } \\
\text { directe ou } \\
\text { vente dans } \\
\text { les huileries }\end{array}$ & 0,7 DT/kg \\
\hline \multirow[t]{4}{*}{ Terrestre } & $\begin{array}{c}\text { Lentisque } \\
\text { Pistacia } \\
\text { lentiscus } \\
\text { (ذرو) }\end{array}$ & $\begin{array}{l}\text { Source } \\
\text { d'énergie pour } \\
\text { la cuisson } \\
\text { du pain }\end{array}$ & & $169 \mathrm{~m} 3 / \mathrm{an}$ & $\mathrm{H}, \mathrm{F}$ & Néant & \\
\hline & $\begin{array}{l}\text { Caroubes } \\
\text { Ceratonia } \\
\text { silica } \\
\text { (خروب) }\end{array}$ & $\begin{array}{l}\text { Consommation } \\
\text { familiale } \\
\text { (gousses) } \\
\text { + vente }\end{array}$ & manuelle & $30 \mathrm{~kg} / \mathrm{an}$ & $H, F, J$ & Néant & \\
\hline & $\begin{array}{l}\text { Câpres } \\
\text { Capparis } \\
\text { spinosa } \\
\text { (كب) }\end{array}$ & $\begin{array}{l}\text { Consommation } \\
\text { familiale } \\
\text { (graines) } \\
\text { + vente }\end{array}$ & manuelle & $\begin{array}{l}350 \mathrm{~kg} / \\
\text { an (2015) }\end{array}$ & $H, F, J$ & $\begin{array}{l}\text { Vente direct } \\
\text { au parc ou } \\
\text { au marché }\end{array}$ & $3 \mathrm{DT} / \mathrm{kg}$ \\
\hline & $\begin{array}{c}\text { Cactus } \\
\text { Opuntia } \\
\text { ficus indica } \\
\text { (هن) }\end{array}$ & $\begin{array}{l}\text { Consommation } \\
\text { familiale (fruits) } \\
\quad+\text { vente }\end{array}$ & manuelle & $1 \mathrm{t} / \mathrm{an}$ & $H, F, J$ & $\begin{array}{l}\text { Au parc ou à } \\
\text { l'entrée du parc }\end{array}$ & $\begin{array}{l}0,8 \text { à } 1,2 \mathrm{DT} \\
\text { la douzaine }\end{array}$ \\
\hline \multirow[t]{2}{*}{ Marais } & $\begin{array}{l}\text { Tamarix } \\
\text { africana } \\
\text { (طرفة })\end{array}$ & $\begin{array}{l}\text { Source } \\
\text { d'énergie pour } \\
\text { la cuisson } \\
\text { du pain }\end{array}$ & branchage & $\begin{array}{l}60 \text { à } 80 \text { kg/ } \\
\text { mois }\end{array}$ & $\mathrm{HF}$ & $\begin{array}{l}\text { Marais de } \\
\text { Melah, Ghezala, } \\
\text { Sejnane }\end{array}$ & $\begin{array}{l}\text { 1,5DT/charge } \\
\text { d'homme }\end{array}$ \\
\hline & $\begin{array}{c}\text { Pelouse } \\
\text { herbacée avec } \\
\text { des scirpes } \\
\text { (المص) }\end{array}$ & $\begin{array}{l}\text { Pâturage ovins } \\
\text { caprins bovins } \\
\text { équidés }\end{array}$ & $\begin{array}{c}\text { Pâturage } \\
\text { direct toute } \\
\text { l'année } \\
\text { (sauf en cas } \\
\text { de montée } \\
\text { des eaux) }\end{array}$ & & $\mathrm{H}$ & & \\
\hline \multirow[t]{2}{*}{ Lac } & $\begin{array}{l}\text { Mulet sauteur, } \\
\text { doré, porc } \\
\text { Lisa saliens, } \\
\text { Aurata, } \\
\text { ramada } \\
\text { (بوري) }\end{array}$ & \multirow[t]{2}{*}{$\begin{array}{l}\text { Consommation } \\
\text { familiale } \\
\text { + vente }\end{array}$} & \multirow{2}{*}{$\begin{array}{l}\text { Par filet sur } \\
\text { les berges } \\
\text { du lac }\end{array}$} & \multirow{2}{*}{$\begin{array}{l}\text { Environ70 } \\
\text { pêcheurs } \\
\text { clandestins } \\
\text { qui réalisent } \\
\text { 200kg par } \\
\text { mois de saison } \\
\text { de pêche }\end{array}$} & \multirow[t]{2}{*}{$\mathrm{H}$} & \multirow{2}{*}{$\begin{array}{c}\text { La vente se fait } \\
\text { dans toutes les } \\
\text { zones de pêche } \\
\text { mais plus } \\
\text { particulièrement } \\
\text { à Tinja et } \\
\text { Menzel } \\
\text { Bourguiba }\end{array}$} & $\begin{array}{c}\text { 7- } 8 \text { DT/ } \\
\text { kg selon la } \\
\text { disponibilité } \\
\text { des poissons } \\
\text { et leurs tailles }\end{array}$ \\
\hline & $\begin{array}{c}\text { Loup / Bar } \\
\text { Dicentrarchus } \\
\text { labrax } \\
\text { (قاروص) }\end{array}$ & & & & & & $20 \mathrm{DT} / \mathrm{kg}$ \\
\hline
\end{tabular}

*H: Homme; F: Femme; J: Jeune
Source: Russiet al. TEEB Wettands, 2013 


\section{Tableau 5 : Les espèces de déclenchement de la zone clé de la biodiversité (ZCB) : Cap Serrat - Cap Blanc - Parc national de I'lchkeul}

\begin{tabular}{lcccc} 
Espèces de & Groupe & $\begin{array}{l}\text { C1 (Espèces } \\
\text { menacés : } \\
\text { CR En danger } \\
\text { critique, } \\
\text { EN En danger, } \\
\text { VU Vulnérable) }\end{array}$ & $\begin{array}{c}\text { C2 (Aire de } \\
\text { répartition } \\
\text { restreinte) }\end{array}$ & $\begin{array}{c}\text { C3 (Communauté } \\
\text { restreinte } \\
\text { à un biome). }\end{array}$ \\
\hline Pleurodeles nebullosus & Amphibiens & VU & NA & NA \\
\hline Anguilla anguilla & Poissons & CR & NA & NA \\
\hline Barbus callensis & Poissons & NA & NA & Oui \\
\hline Anodonta lucasi & Mollusques & CR & Oui & NA \\
\hline Gomphus lucasii & Odonates & VU & NA & Oui \\
\hline Bellis prostrata & Plantes & NA & Oui & NA \\
\hline Pilularia minuta & Plantes & EN & NA & NA \\
\hline Rumex tunetanus & Plantes & CR & Oui & NA \\
\hline Serapiass tenopetala & Plantes & CR & Oui & NA \\
\hline
\end{tabular}

Source : Darwall et al, 2015

3.3 Identification des bénéfices économiques indirects fournis par le PNI

II s'agit des services de régulation qui constituent des valeurs La dégradation de l'écosystème entraine aussi des coûts d'usage indirect (cf. Tableau 6). IIs contribuent à la protection économiques. Par exemple, la perte des marais peut causer des gens, des animaux, de la production agricole des terres le déplacement des oiseaux pour se nourrir dans les terres périphériques et des biens matériels.
3.4 Identification des bénéfices potentiels

II s'agit de la valeur d'option liée à des opportunités d'usage tibles d'être développés au PNI, nous pouvons retrouver pluactuelles ou futures qui pourraient être tirées des écosys- sieurs activités d'écotourisme possibles telles que la possitèmes de l'Ichkeul sans compromettre les objectifs de bilité d'organiser des visites guidées, la pratique du vélo tou conservation (cf. Tableau 7).

Aussi, le nombre d'utilisateurs potentiels des eaux thermales pour des usages therapeutiques, nettoiement, soins, etc. est très important selon les attentes exprimées par la population riveraine et régionale.

Par ailleurs, les plantes (et la biodiversité en général) peuven être composées de produits pharmaceutiques et autres ressources génétiques, encore inconnus, et qui feront l'objet d'une demande future.

Le parc présente des fortes opportunités de récréation et d'éducation environnementale, en effet, il est situé à environ $75 \mathrm{~km}$ du Grand Tunis (2,64 million d'habitants) et à $25 \mathrm{~km}$ de Bizerte (560.000 habitants). Il est bien desservi par les routes et l'autoroute Tunis-Bizerte. Le nombre de visites peut varier en fonction du degré de promotion au niveau des médias et des agences de voyage, le degré d'information des visiteurs potentiels, des services fournis et le ciblage des visiteurs
potentiels, notamment les ornithologues. Si l'on ajoute les potentiels, notamment les ornithologues. Si l'on ajoute les visites éducatives (classes vertes) et les visites nature (pour l'observation des oiseaux en hiver), le nombre de visites peut atteindre 100.000 personnes par an. II faut mentionner que le nombre de visite annuel au parc de la Camargue en France, qui est aussi un site d'accueil pour les oiseaux, est de 1 million de visiteurs. Concernant les services susceperrain, de la spéléologie, des excursions dans les douars (villages de tentes) et les villages locaux et du trekking sportif sur le Djebel Ichkeul, lequel culmine à 500 mètres au-dessus du lac et offre de superbes vues panoramiques des marais, de visites des vestiges romains. II est également possible de faire des excursions plus longues à dos de cheval ou de chameau aux abords du lac et de bivouaquer sur ses rives (RAMSAR, 2012).

Toutefois, le plan de gestion des visiteurs du Parc national d'lchkeul (UE, du parc plus faible (43335 visiteurs par an)

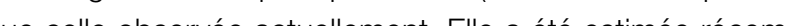
ment pour les différentes activités de pac en enployantun-

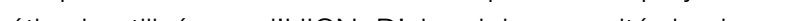
physiqu (COP) a fé estimée en se bas an surte do charge phy visteus a le circult peut accuellir physiquement, ensulte, a capacité de charge réelle (CCR) a été calculée en estimant le nombre maximum de visiteurs admissibles sur un cicuit, tenant compte des parametres biophysiques, envionnementaux (insolation, précipitation), sociaux (nombre de personnes par groupe, distance entre les groupes) $)^{8}$. Cette estimation de capacité de charge à une centaine de visiteurs par jour peut paraitre très faible par rapport à la constatation lors d'une visite effectuée le 20 décembre 2015, avec la présence de plus de 1000 visiteurs.

\section{Tableau 7 : Liste des bénéfices potentiels du Parc National I chkeul}

\section{Tableau 6 : Liste des bénéfices directs fournis par le Parc National Ichkeul}

\section{Service de régulation}

Rétention des sols

Forêts

Séquestration du carbone

Contrôle des inondations

Marais et lac

Rétention des sédiments

Recharge de la nappe

\section{Benefice}

Prévention de l'érosion et réduction

Régulation du clima

Protection contre les inondations des terres de cultures et de la ville

Réduction des sédiments dans les barrages et augmentation de leurs capacités d'eau

Utilisation de l'eau pour l'eau

potable et l'irrigation

Contribution a la régulation du microclimat
Opportunités de récréation futures

Forêts / marais / lac

\section{Opportunités d'éducation environnementale futures}

Opportunités de visite pour les amateurs de la nature et de l'ornithologie

Opportunités de l'usage des eaux thermales à des fins thérapeutiques

Opportunités de l'usage pharmaceutique de certaines plantes

"La forte différence entre CCP et CCR laisse penser que la CCR a été sous-estimée en utilisant des hypothèses

de calcul non réalistes, par exemple, une distance de $250 \mathrm{~m}$ doit séparer deux groupes de visiteurs:
- Hammam Ben Abbès : CCP: 6212 visiteurs par jour et un CCR de 67 visiteurs par jour en moyenn

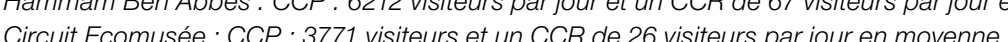

- Hammam Negrez : CCP - 2756 visiteurs et un CCR de 26 visiteurs par jour en moyenne. 
Dans le même rapport cité précédemment. Aouididi (1996) sortant suffisant. Toutefois, les relations de cause à effet sont a établi une fonction linéaire entre la variation de la pêche difficiles à établir (ANPE, 2006).

des poissons de la fanill Muges dores) et la varilion de a silité à parir dos observations effectuées entre 1982 et $1995^{\circ}$. Cette variation est supposee être la même relativement aux autres espèces migratrices telles que les Anguilles, Soles ou Loups. Toutefois, l'évolution de la production sur la période 1996-2015 a montré une résilience de l'écosystème face aux changements environnementaux subis (épisodes sèches / humides). Selon M.S. Romdhane (communication personnelle), c'est plutôt le niveau d'eau qui affecte la production de poissons et non pas la salinité. Les poissons peuvent supporter une salinité de $40 \mathrm{~g} / \mathrm{l}$, mais, les forts courants d'eau pourraient affecter la production de poissons avec un décalage qui peut aller jusqu'à 7 ans. La variation de la production peut être axpliqúe plutot par leftort de peche. Touletos, in n'y a pas de corrélation entre la quantité des apports en eau au lac et de correlation entre la quantite des apports en eau au lac et
la production de poissons sur la période 2001 et 2014 (cf. Figure 4). II n'y a pas de baisse observée de la production de Figure 4). II n'y a pas de baisse observee de la production de.
poissons (t) durant les années à faible apport d'eau douce. poissons (t) durant les annees à faible apport d'eau douce. est possible que la sècheresse affecte negativement la pro-
duction avec un décalage de 1 à 3 ans, voire plus, avec des mauvaises conditions de recrutement faute de courant d'eau

Il est communément admis que la conservation de la biodiversité constitue un support pour la fourniture des services ecosystémiques. Dans le cas du PNI, plusieurs auteurs ont souligné le lien entre la biodiversité et le fonctionnement de 'écosystème, notamment les effets négatifs de la baisse du niveau d'eau du lac et de sa salinité sur la biodiversité a du mper ed ses pas eu de fortes baises des flux des sevices (poiss in pas en da for es basses des flux des services (poissons, 2000-2015 magre les events de sechnt la periode 2000-2015 malgre les evenements de secheresse. II est probable que la resilince de lecosysteme et les efforts de preservation du parc ont joue un role positif dans ce sens. Daily (1997) souligne le faible effort de recherche sur le lien entre le fonctionnement de lecosysteme et la provision eservices, le probleme majeur etant que les processus (eosystemiques sont souvent mesures a des echelles spatiales differentes par rapport aux services ecosystemiques. y a donc un besoin de développer la recherche pour letablissement de modèles intégrant à la fois les aspects les modèles devront être appliqués pour les pratiques de gestion existantes, ainsi que pour les scénarios de gestion étudiés.

Figure 4 : Relation entre la production de poissons et la quantité des apports en eau au lac (DG Pêche, ANPE)

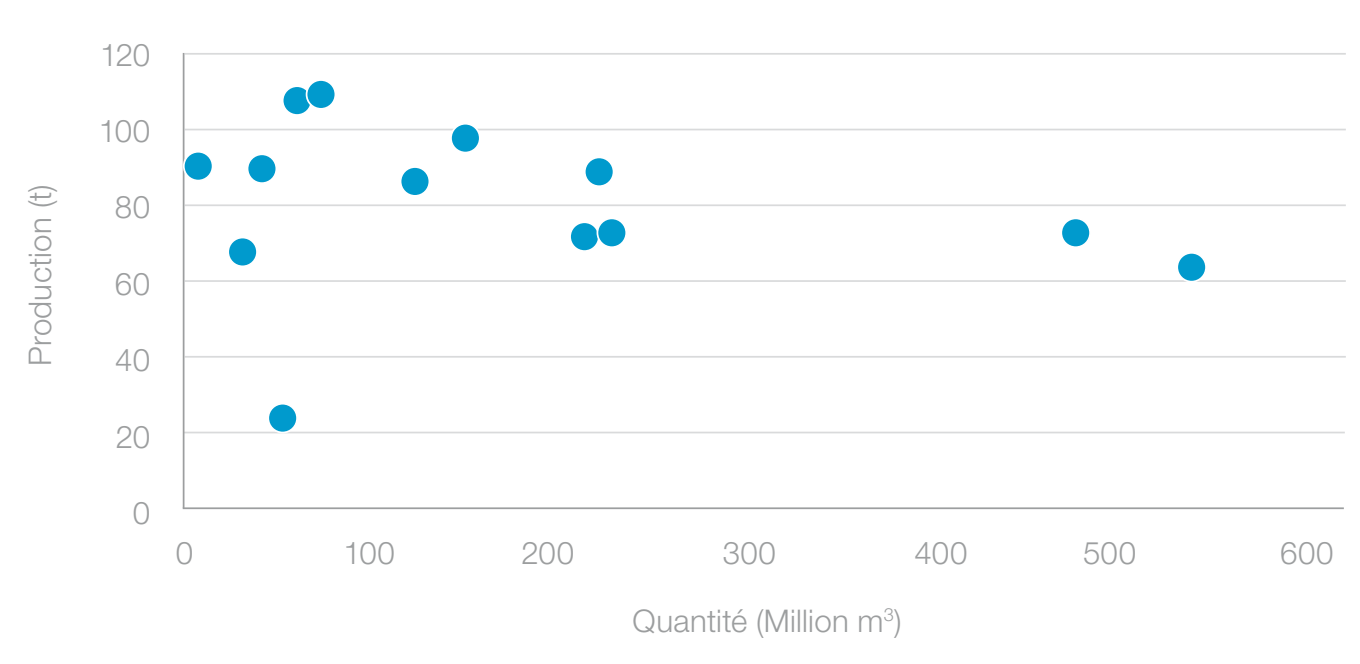

"Comme suit: $\Delta H=-E^{*} 1.2424 \Delta S$; avec $\Delta H$ étant la variation de la quantité de poissons pêchée, E, étant l'effort de pêche lle nombre un effort de pêche estimé à 348 sorties par an a entrâné une perte de 2594 kg (2.7\%) de poissons pêchés Aoudidid 1996)

\section{4}

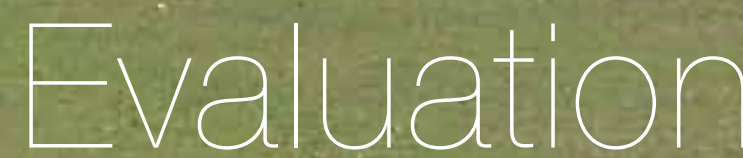

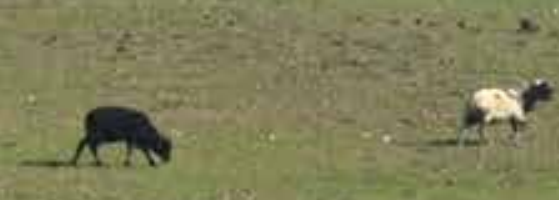
$000 \Omega 0$ $\theta 0 S \Omega S R$ $\therefore 0000000$
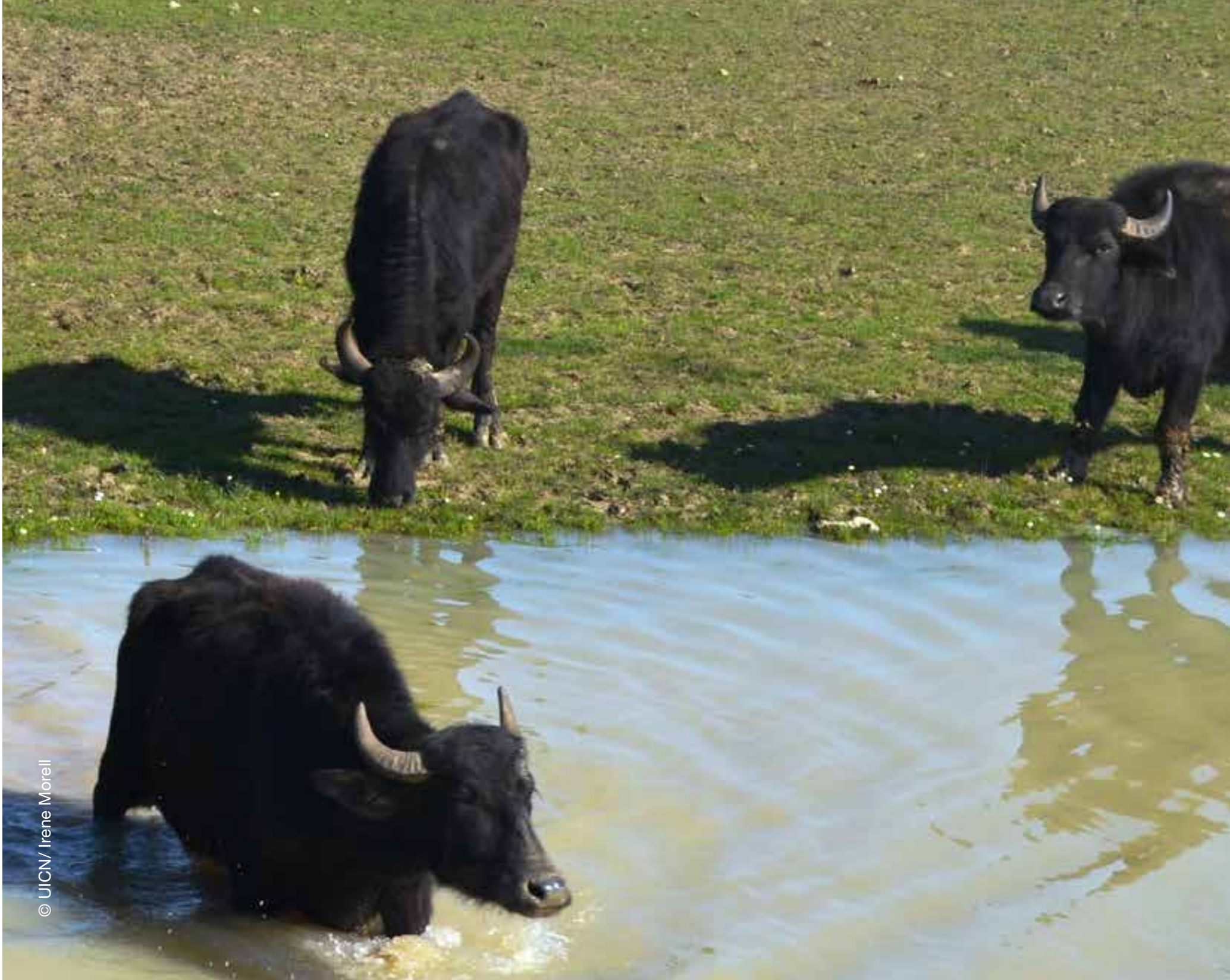
Cette section présente en détail les valeurs économiques des bénéfices directs et indirects fournis par le Parc National de l'Ichkeul, ainsi qu'une comparaison des résultats avec ceux des évaluations similaires.

\subsection{Valeurs économiques des bénéfices directs}

C'est la population locale qui profite d'un certain nombre de lation habitant à l'intérieur du parc est celle de 50 à 64 an bénéfices d'usage direct: pâturage, olives, câpres, caroubes, avec 39,2\% suivie de celle des plus de $65 a n s$ avec $27,5 \%$ bois services lís au hammam. En l'absence de données sur celle de 35 à 49 ans avec $25.5 \%$, tandis que pour la popu-

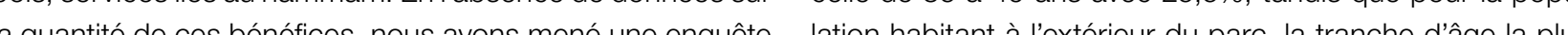

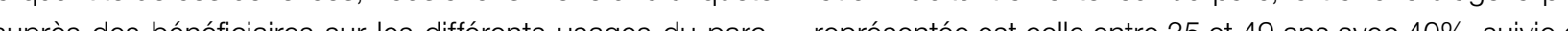

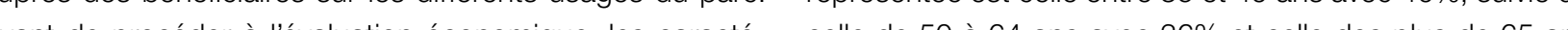
et leur - avec $20 \%$ (Tableau 8 .

cont décrites.

Les plus jeune (-35ans) sont les moins présent dans les deux

Caractéristiques socio-économiques et perception du catégories avec 7,8\% dans l'échantillon de la population lo-

\section{parc par la population locale}

Lenquête a été réalisée auprès de la population locale du Parc National Ichkeul, et les questions ont été posées à 100 chefs de ménage avec une distinction entre les habitants de lintérieur (50) (48\% de l'ensemble des ménages) et de l'extéreur du parc (50) (26\% de l'ensemble des ménages).

Les chefs de ménage interrogés sont exclusivement de sexe masculin, d'âge moyen de 54 ans pour la population interieure et de 52 ans pour la population de lexterieur. La rranche d'âge la plus présente dans l'échantillon de la popu-

(io cale de linterieur du parc et $4 \%$ de celle de l'extérieur. La aille moyenne des ménages des deux catégories est très similaire, avec 4,7 pour les habitants de l'intérieur et 4,5 pour ceux de l'extérieur. La taille de ménage varie entre 1 et 9 personnes par ménage pour toute la population locale.

La majorité des enquêtés ayant répondu (40\% pour l'intérieur du parc et $34 \%$ pour l'extérieur) ont un niveau d'études primaire, alors que $28 \%$ des habitants sont des analphabètes. Seulement $11 \%$ des personnes ont un niveau d'études secondaires et $2 \%$ d'entre eux ont un niveau d'étude supérieur (cf. Tableau 9).

Tableau 8: Répartition des effectiffs selon lâge (Interieur et Extérieur du parc) (années)

\begin{tabular}{|c|c|c|c|c|}
\hline & \multicolumn{2}{|c|}{ Intérieur } & \multicolumn{2}{|c|}{ Extérieur } \\
\hline Moyenne & \multicolumn{2}{|c|}{53,6} & \multicolumn{2}{|c|}{51,7} \\
\hline Ecart-type & \multicolumn{2}{|c|}{13} & \multicolumn{2}{|c|}{14,6} \\
\hline Min & \multicolumn{2}{|c|}{28} & \multicolumn{2}{|c|}{24} \\
\hline \multirow[t]{2}{*}{ Max } & \multicolumn{2}{|c|}{80} & \multicolumn{2}{|c|}{91} \\
\hline & Nombre & Pourcentage & Nombre & Pourcentage \\
\hline $24-34$ ans & 4 & $7,8 \%$ & 2 & $4 \%$ \\
\hline 35 - 49ans & 12 & $25,5 \%$ & 20 & $40 \%$ \\
\hline 50 - 64ans & 20 & $39,2 \%$ & 18 & $36 \%$ \\
\hline$>65$ ans & 14 & $27,5 \%$ & 10 & $20 \%$ \\
\hline
\end{tabular}

Tableau 9 : Répartition des effectif́s selon le niveau d'instruction (Intereieur et Extérieur du parc)

\section{Analphabèt}

Primaire

Secondaire

Supérieur

Pas de réponse

\section{Niveau d'instruction}

Intérieur

Nombre

14

(14)
$28 \%$

$40 \%$

$12 \%$

$2 \%$
Extérieur

Nombre

14

5
Pourcentage

$28 \%$

$34 \%$

$10 \%$

$2 \%$
Tableau 10 : Répartition des effectifs selon la profession exercée (Intererieur et Extérieur du parc)

\begin{tabular}{lcccc}
\multicolumn{1}{c}{ Profession } & \multicolumn{2}{c}{ Intérieur } & \multicolumn{2}{c}{ Extérieur } \\
\hline Agriculteur/Eleveur & 12 & Pourcentage & Nombre & Pourcentage \\
Retraité & 9 & $24 \%$ & 14 & $28 \%$ \\
Sans Emploi & 1 & $18 \%$ & 6 & $12 \%$ \\
Employé & 1 & $2 \%$ & 0 & - \\
\hline Ouvrier/Gardien du parc & 15 & $2 \%$ & 1 & $2 \%$ \\
\hline Ouvrier/Gardien à & 12 & $30 \%$ & 12 & $24 \%$ \\
l'extérieur du Parc & & $24 \%$ & 17 & $34 \%$
\end{tabular}

La répartition de l'échantillon en fonction des activités pro- vage sont pratiqués par $28 \%$ de l'échantillon alors que $24 \%$ fessionnelles, a été faite non seulement sur les résultats travaillent comme ouvrier ou gardien dans le parc.

de l'enquête mais aussi en ayant recours à une distinction entre les activités à l'intérieur du Parc et celle de l'extérieur pour les deux groupes étudiés (Intérieur et Extérieur du parc) (cf. Tableau 10).

En termes de revenu, le revenu annuel par ménage est estimé à 11.929 DT à l'intérieur et 16.715 DT à l'extérieur du parc. Ce revenu est largement inférieur au niveau de vie du pays. En effet, le PIB par menage en 2014 est de 29.789 DT (INS) Pour les habitants du parc, les activités les plus exercées Le taux de pauvreté de la population locale (en utilisant un sont en relation directe avec le parc, à savoir ouvrier ou seuil de revenu de 1.500 DT/habitant) est similaire à l'intérieur garde forestier dans le parc (30,6\%), agriculture et élevage du parc (35\% des ménages) et à l'extérieur (34\%). La popu(24,5\%). Les individus exerçant des activités professionnelles lation locale génère une partie importante de son revenu à à l'extérieur du parc (22.5\%) sont soit des ouvriers, des gar- partir des activités liées aux usages des ressources natudiens ou encore des journaliers. Seulement une personne relles du parc. En premier lieu l'élevage basé sur le pâturage interrogée est employée. En ce qui concerne le deuxieme groupe etudie (les habitants de l'exterieur du parc), 34\% exercent des professions à l'exterrieur du parc; à savoir le métier d'ouvrier, gardien, journalier. Seulement une personne interrogée est employée, ainsi an l'extel successivment pour la population de lintenteur et de que $12 \%$ des interrogés sont retraitées. L'agriculture et l'éle- l'exterieur. Lapiculture, la pêche illégale et l'exploitation des produits forestiers génèrent des faibles revenus (Tableau 11). 
Tableau 11 : Distribution des revenus de la population locale

\begin{tabular}{|cccccccc} 
Elevage & Apiculture & $\begin{array}{c}\text { Production } \\
\text { agricole }\end{array}$ & Pêche & $\begin{array}{c}\text { Salaires } \\
\text { intérieur } \\
\text { du parc }\end{array}$ & $\begin{array}{c}\text { Salaires } \\
\text { relation } \\
\text { avec le parc }\end{array}$ & $\begin{array}{c}\text { Salaires } \\
\text { hors du } \\
\text { parc }\end{array}$ & $\begin{array}{c}\text { Exploitation } \\
\text { des produits } \\
\text { forestiers }\end{array}$ \\
\hline $43 \%$ & $2 \%$ & $3 \%$ & $3 \%$ & $28 \%$ & $9 \%$ & $12 \%$ & $0,3 \%$ \\
\hline $26 \%$ & $2 \%$ & $18 \%$ & $0 \%{ }^{10}$ & $22 \%$ & $4 \%$ & $28 \%$ & $0,1 \%$ \\
\hline
\end{tabular}

La majorité des habitants de l'intérieur du parc interrogés faible pluviométrie (23\%) (Tableau 13). Les résultats obtenus (98\%) considèrent le parc comme habitat, aussi, $96 \%$ le auprès des habitants à l'extérieur du parc concernant leurs considèrent comme source de revenu. Aussi, $86 \%$ des ha- avis sur la situation du parc sont très proches des résultats bitants de l'extérieur considèrent le parc comme source de précédemment présentés. Ainsi $78 \%$ qualifie l'́tat du parc de revenu et $18 \%$ considrent le parc comme un espace de bon à loisirs (Tableau 12). Pour la totalité des chefs de ménage in- dis que $16 \%$ le trouvent en dégradation du principalement au terviewés de l'intérieur du parc, leurs relations avec le milieu surpâturage. La totalité de la population locale (vivant à l'inenvironnant est "Très fortes"; ; cette relation provient princi- térieur ou à l'extérieur du parc) entretiennent de très bonnes palement de la présence de la montagne, des marais, du relations avec les équipes chargées de la gestion du parc. lac et des sources d'eaux thermales. En ce qui concerne La création du parc a induit une restriction des usages des les chefs de ménage qui habitent à lexterieur du parc, bien ressources fournis par le parc (cf Chennoufi 1995a et Chenqu'ils soient d'accord avec les habitants de linterieur sur noufi, 1995b). La récolte du bois ne concerne plus que l'importance accordé à leurs relations avec le milieu environ- bois mort. De même, la chose est interdito l'oxploitation nant, ils ont une perception différente de la provenance de cette importance. Ainsi, cette relation provient des marais, du lac, et dans une moindre mesure de la montagne et des lette dos grains drolastres et do capros vendus principasources d'eau thermale. D'après l'échantillon étudié, le degré lement à létat brut. Par ailleurs, les familles a l'interieur du d'attachement des enfants au parc est très fort pour 98\% parc disposent de jarlins situes tout près des habitations. des habitants de l'intérieur du parc et pour 96\% des habi- Elles ont une superficie moyenne de 7 ares et sont occupées tants de l'extérieur. essentiellement par des cultures potagères et par quelques arbres fruitiers (grenadier, amandier) dont le produit est desLa majorité des individus participants à l'enquête et habi- tiné exclusivement à l'autoconsommation familiale (PDC). Le tant à l'intérieur du parc (70\%), qualifie l'état du parc de bon poisson est le principal produit marchand du parc. A partir à très bon, 4\%qualifient l'état plutôt de stationnaire, tandis de 1998, une concession pour l'autorisation de pêche a été que $26 \%$ le trouvent en dégradation. Plusieurs causes de accordée à un opérateur privé "Tunisie Lagunes " contre dégradation ont été évoquées, dont principalement "le sur- une redevance de 210.000 dinars/an.

pâturage" (92\%) "Ia non application ou le non-respect des règles" (38\%) ou encore des causes naturelles telles que la
Tableau 12 : Importance des différentes composantes du milieu environnant

\begin{tabular}{lcccc}
\hline Composantes de l'écosystème & \multicolumn{2}{c}{ Population à l'Intérieur } & \multicolumn{2}{c}{ Population à l'Extérieur } \\
& Nombre & Pourcentage & Nombre & Pourcentage \\
\hline Les sources d'eau thermale & 46 & $92 \%$ & 33 & $66 \%$ \\
\hline Montagne & 50 & $100 \%$ & 34 & $68 \%$ \\
\hline Marais & 48 & $96 \%$ & 48 & $96 \%$ \\
\hline Lac & 46 & $92 \%$ & 38 & $76 \%$ \\
\hline Total & 50 & & 50 &
\end{tabular}

\section{Tableau 13 : Etat du parc d'après la population locale}

\begin{tabular}{lcccc}
\multicolumn{1}{c}{ Etat du parc } & \multicolumn{2}{c}{ Population à l'I'ntérieur } & \multicolumn{2}{c}{ Population à l'Extérieur } \\
& Nombre & Pourcentage & Nombre & Pourcentage \\
Bon à Très Bon & 35 & $70 \%$ & 39 & $78 \%$ \\
Stationnaire & 2 & $4 \%$ & 3 & $6 \%$ \\
En dégradation & 13 & $26 \%$ & 8 & $16 \%$ \\
Total & 50 & & 50 &
\end{tabular}

\section{Valeurs économique des différents usages des res-} sources par la population locale

Bois

Selon l'enquête effectuée auprès de la population vivant

lintérieur du parc, $76 \%$ des ménages pratiquent la collecte de bois pour la préparation du pain traditionnel. L'estimation de la consommation par ménage utilisateur varie entre 0,21 et 2,7 t/an, avec une moyenne de 1,1 t/an. Au total, le prélèvement de bois est estimé à $169 \mathrm{~m}^{3} / \mathrm{an}$, beaucoup plus faible par rapport à l'accroissement de la biomasse forestière, estimé à $545 \mathrm{~m}^{3} / a n$ (cf. Tableau 14). De plus, le volume du bois mort est estimé à 30 stères. Avec un prix moyen de $10 \mathrm{DT} / \mathrm{m}^{3}$ de bois de maquis sur pied, la valeur du bois est estimée à 1.690 DT/an.
Autres produits forestiers

La recolte des grains d'oleastres est effectuée en fonction de la taille des grains surtout durant les annees de forte production. La récolte moyenne en 2015 est estimée à 182 $\mathrm{kg} /$ menage. En effet, la faible productivité et le faible renants) en huile $(9 \%$ de taux d'huile selon certains habitants) n'incitent pas à la récolte. La récolte de câpres est kgsiménectuee en cas de forte production, elle etait de la consonmation local La récolte de caroubes est négrgeable, selenent $8 \%$ le pratiquent pour une production

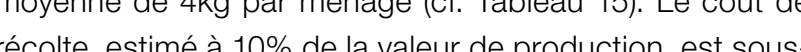
trait de la valeur de production. moyenne de 4kg par mén pratige (cf. Tablu une production 
Tableau 14 : Potentiel de production et prélèvements de bois de la forềt du PNI

\begin{tabular}{|c|c|c|c|c|}
\hline Surface (ha) & $\begin{array}{l}\text { Accroissement } \\
\text { annuel en bois } \\
\left(\mathrm{m}^{3} / \mathrm{ha} / \mathrm{an}\right)\end{array}$ & $\begin{array}{l}\text { Accroissement } \\
\left.\text { en bois ( } m^{3} / a n\right)\end{array}$ & $\begin{array}{l}\text { Prélèvement de } \\
\left.\text { bois ( } \mathrm{m}^{3} / \mathrm{an}\right)\end{array}$ & Valeur (DT/an) \\
\hline
\end{tabular}

Forêts

1363 0,4
169

1690

Tableau 15 : Potentiel de production et prélèvements de produits forestiers non ligneux de la forêt du PNI

\begin{tabular}{lccccccc} 
Produits & $\begin{array}{c}\text { Surface } \\
\text { exploitable (ha) }\end{array}$ & $\begin{array}{c}\text { Production } \\
\text { Potentielle (t/an) }\end{array}$ & $\begin{array}{c}\% \\
\text { ménages }\end{array}$ & $\begin{array}{c}\text { Q moyenne } \\
\text { kg/ménage }\end{array}$ & $\begin{array}{c}\text { Qen 2015 } \\
\text { (t/an) }\end{array}$ & $\begin{array}{c}\text { Prix } \\
\text { (DT/kg) }\end{array}$ & $\begin{array}{c}\text { Valeur } \\
\text { (DT/an) }\end{array}$ \\
$\begin{array}{l}\text { Graines } \\
\text { d'oléastres }\end{array}$ & 500 & 250 & $75 \%$ & 182 & 14,2 & 0,700 & 9926 \\
$\begin{array}{l}\text { Câpres } \\
\text { Caroubes }\end{array}$ & 50 & 6 & $55 \%$ & 6,1 & 0,35 & 3 & 1047 \\
$\begin{array}{l}\text { Fruits de } \\
\text { cactus }\end{array}$ & 5 & 3 & $8 \%$ & 4 & 0,03 & 1,5 & 50 \\
\hline
\end{tabular}

\section{Nectar}

L'apiculture n'est pas une activité très pratiquée dans le parc malgré l'opportunité en termes de revenu qu'elle procure et la qualité du produit (miel biologique) qu'elle peut génére. En effet, 16\% de ménages l'exercent, ils possèdent entre 2 et 30 ruches chacun (avec une moyenne de 16 ruches par ménage) ; la production moyenne est de $5 \mathrm{~kg} /$ ruche, le prix étant de $30 \mathrm{DT} / \mathrm{kg}$ et le revenu net moyen par ménage utilisateur est estimé à $1.447 \mathrm{DT} /$ an selon l'enquête. Au total, a valeur nette relative à la production du nectar est estimée à travers la multiplication du revenu net par ménage par le nombre de ménages utilisateurs, soit 24.078 DT/an.

Fourrages

Lélevage est la principale activité de la population locale habitant à l'intérieur du parc (80\% des ménages selon l'enquête). Le calendrier fourrager est à base de pâturage dans tée du niveau d'eau), des chaumes des céréales (juillet-Aout) et d'une alimentation de complémentation à base d'orge grain ou de son de blé $(0,5$ à $1 \mathrm{~kg}$ par tête et par jour), de foin te de paille (septembre-février) (CRDA Bizerte 2006). Selon notre enquête, le nombre d'animaux appartenant à la population à lintérieur du parc est estimé à 3.208 (371 bovins, 2.355 ovins et 481 caprins).

La population locale vivant à l'extérieur du parc exerce moins activité élevage que la population à l'intérieur du parc, touefois, le nombre d'animaux reste assez élevé, il est estime 3.640 (671 bovins, 2.902 ovins et 67 caprins). Le nombre d'animaux total pâturant dans le parc (6.847) est plus faible que celui de 1988 (8.770 animaux comptabilisés dans les maais selon Thomas et al. 1991). La forte charge animale entaine une dégradation des parcours collectifs (surpâturage) entrainant un recours plus important à l'achat des aliments de bétail (CRDA Bizerte 2006). Le tableau 16 indique le nombre néfice net dépend de la race et de la pratique de l'élevage.
Tableau 16 : Nombre d'animaux et bénéfice net (en DT) de l'élevage exercé par la population à lintérieur et à l'extérieur du parc

\begin{tabular}{llcccc} 
& & Bovin & Ovin & Caprin & Elevage \\
\hline$\%$ & Intérieur & $41 \%$ & $59 \%$ & $41 \%$ & \\
$\%$ & Extérieur & $39 \%$ & $47 \%$ & $6 \%$ & \\
\hline $\begin{array}{l}\text { Nombre moyen de } \\
\text { tête par éleveur }\end{array}$ & Intérieur & 10 & 42 & 12 & \\
\cline { 2 - 3 } & Extérieur & 9 & 33 & 6 & \\
/tête par éleveur & Extérieur & 490 & 236 & 186 & \\
$\begin{array}{l}\text { Bénéfice net moyen / } \\
\text { an par éleveur }\end{array}$ & Intérieur & 837 & 314 & 127 & \\
& Extérieur & 4912 & 8617 & 2153 & 9082 \\
\hline
\end{tabular}

Tableau 17 : Estimation de la production fourragère du PNI

\begin{tabular}{lcccc} 
& S (ha) & $\begin{array}{c}\text { Production } \\
\text { fourragère (UF) }\end{array}$ & $\begin{array}{c}\text { Valeur brute de } \\
\text { la production } \\
\text { fourragère (DT) }\end{array}$ & $\begin{array}{c}\text { Valeur nette de } \\
\text { la production } \\
\text { fourragère (DT) }\end{array}$ \\
\hline Forêt & 1340 & 194761 & 97380 & 80825 \\
\hline Marais & 2737 & 1368500 & 684250 & 567927 \\
\hline Total PNI & & 1563000 & 781630 & 648753
\end{tabular}

L'estimation de la valeur économique du pâturage est basée Nettoyage, relaxation et soin au Hammam sur la production fourragère. La productivite fourragère des (Bain chaud traditionnel)

forêts est estimée par le plan d'aménagement et de gestion Les sources thermales à proximité sont fermées. II n'y a que (DGF 2006). Elle varie de 100 à 500 UF/ha/an selon le type le Hammam Negrez qui est utilisé actuellement, ce hammam de végétation avec une moyenne de $145 \mathrm{UF} / \mathrm{h}$ /an. Celle des est assez lointain et nécessite un bon effort physique pour marais est estimée à $500 \mathrm{UF} / \mathrm{ha} / \mathrm{an}$ (communication avec Mr $\begin{aligned} & \text { est assez lointain et nécessite un bon effort physique pour } \\ & \text { s'y rendre. Selon l'enquête, } 90 \% \text { des ménages de lintérieur }\end{aligned}$ H. Ghazouani). La valeur de l'unité fourragère est estimée à s'y rendre. Selon l'enquête, $90 \%$ des menages de linterieur travers le prix du produit de substitution, l'orge, soit 0,5 DT/kg. $\begin{aligned} & \text { du parc fréquentent le hammam } 32 \text { fois par an en moyenne } \\ & \text { (2,6 fois par mois) et } 59 \% \text { des ménages de l'extérieur le fré- }\end{aligned}$ Selon les données de l'enquête, le coût de main d'œunre pour (2,6 fois par mois) et $59 \%$ des ménages de l'extérieur le frégarder le troupeau (berger) correspond à $17 \%$ en moyenne de quentent 30 fois par an avec les membres de la famille $(2,5$ la valeur du bénéfice net de lélevage. Ce coût est soustrait de fois par mois). Au total, le nombre de visites au hammam est la valeur fourragère. La valeur économique du pâturage rieur (Tableau 18). Toutefois, cette fréquentation peut causer est ainsi estimée à 648.753 DT (cf. Tableau 17). 
Tableau 18 : Fréquentation du Hammam par la population locale

\begin{tabular}{lccc} 
& $\begin{array}{c}\text { \% des ménages } \\
\text { utilisateurs }\end{array}$ & $\begin{array}{c}\text { Nombre moyen par } \\
\text { ménage par an }\end{array}$ & Membres impliqués \\
\hline Population à l'intérieur du parc & $90 \%$ & $111(8-324)$ & $H(94 \%), F(69 \%), \mathrm{J}(65 \%)$ \\
\hline Population à l'extérieur du parc & $59 \%$ & $57(5-576)$ & $H(59 \%), \mathrm{F}(37 \%), \mathrm{J}(33 \%)$
\end{tabular}

La valeur économique est estimée au minimum égale au prix La quantité de production publiée officiellement pourrait d'entrée au hammam ordinaire, soit 1,5 DT, sans tenir compte être inférieure à la production réelle à cause de la sous-dèdes qualités thérapeutiques. Plusieurs personnes enquêtées claration (pour des problèmes de contrôle et de suivi) et la ont exprimé leur disposition à payer ce montant. Ainsi, la pêche illégale par la population locale qui pourrait atteindre valeur économique attribuée au nettoyage, relaxation et quelques tonnes selon la STL. Selon des entretiens avec cersin est estimée à 25.168 DT Autrement, la méthode de tains habitants de la région, environ 25 personnes exercent coût de remplacement pourrait être utilisée pour l'évaluation. cette activité malgré leurs arrestations répétitives.

La ferme des habitants du parcves Ies centes ubans voisns pourse baigner (CRDA Bizerte, 2006), soit un coût moyen de 20 DT/ trajet, ou 5 DT/personne environ

\section{Poissons}

La Société Tunisie Lagunes (STL) assure l'exclusivité de la pêche dans le lac Ichkeul, suite à une concession avec l'APAL, toutefois, le montant de la concession (210 .000 DT/an initialement) n'est pas payé réellement à l'Etat. Cette société emploie actuellement une trentaine d'ouvriers de la localité de Tinja et deux habitants du parc (CRDA Bizerte 2006). La valeur économique des poissons est basée sur le prix de marché. Les données sur la production sont publiées par l'Annuaire statistique de la DG Pêche.

La moyenne de la production (2000-2011) est de 80 t/an environ, si l'on exclut la baisse liée à des facteurs conjoncturels 2012-2013). La production des anguilles représente environ $30 \%$ de la production totale (selon les donnees du concessionnaire). Environ 90\% de cette production est destinee lexportation, tandis que les autres $10 \%$ sont destinés au marché national. Le prix moyen des anguilles vivantes à l'exportation était de 17,2 DT/kg en 2014".

Par ailleurs, une partie des poissons élevés à Ichkeul pourrait être pêchée au lac de Bizerte, mais sa quantification en terme de gain de poids, abondance, etc. est très difficile (DG Pêche). En effet, le lac Ichkeul constitue un milieu très favorable pour la croissance des alevins, qui passent un à deux ans dans le lac avant qu'ils atteignent la maturité sexuelle migrent vers le lac de Bizerte et la mer.

La variation annuelle des prélèvements (cf. figure 5) est attribuee essentiellement (i) aux annees de secheresse, au comblement du lac provoqué par les déversements des oueds par les barrages et (iii) à une évaporation excessive entrainant une diminution significative de la profondeur du ac (0,50 $\mathrm{m}$ en été). Cette faible profondeur a comme conséquence (i) une augmentation de la température de l'eau, (it) de la salinité et (iii) une diminution de l'oxygène. Ce dysfonc-

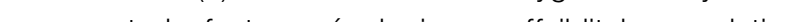
piscicole (ains que les Potamots et les Scirpes) qui dispat progressivent. Le phén des Scipes) avi disparat du lac, conón. Le phenomene deutrophisation des eaux nements, aggrave encore la situation (DGF 2006).

En 2014, la production de poissons est estimée à 97,7 t, sa valeur brute est estimée à 752.535 DT (Tableau 19). Si l'on soustrait le coût de l'exploitation estimé à 26.000 DT/mois (estimation effectuée selon le nombre de salariés (32) et le salaire mensuel moyen de $800 \mathrm{DT} T^{12}$ ), la valeur nette des poissons est estimée à $440.535 \mathrm{DT}$.

$11 /$ convient de noter que le prix des anguilles à l'étranger est plus cher, les adultes peuvent atteindre $20 € / \mathrm{kg}$ sur le marché en Espagne avec un (3) ${ }_{12}^{2}$ Nous n'avons pas pu avoir les données de la part de la STL malgré les nombreuses prises de contact.

Figure 5 : Evolution de la production des poissons dans le lac lchkeul (en t) (DG de la Pêche)

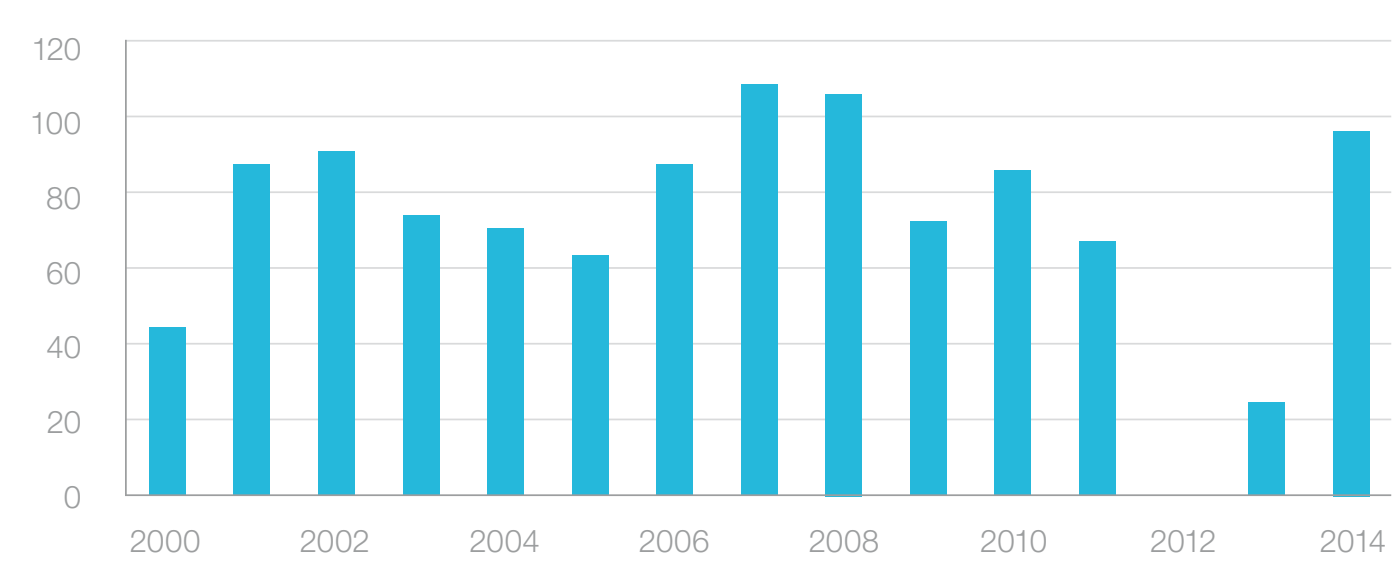

Chasse

La chasse est autorisée au niveau de la zone périphérique, elle est cependant interdite au niveau d'une frange ripicole d'une centaine de mètres à partir des limites du lac. L'autorisation de chasse est règlementée par la DGF. Elle concerne les oiseaux d'eau tels que les bécassines, les bécasses, les perdreaux, et certains canards. Des battues pourraien être également autorisées dans la zone périphérique sous contrôle de l'Arrondissement des Fôts contre un paiement de redevances, aux chasseurs nationaux possédant un permis de chase. Eles concernent des especes telles que les

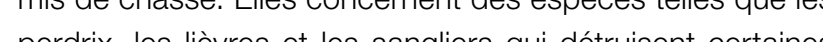
peranx, les lievros et les sanglers qui dectrisent certaines tivités. tivités de chasse dans la periphene du parc en 2015. Toutefois, nous ne disposons pas diinformations sur la chass illégale (pour les canards notamment).

Tableau 19 : Estimation de la valeur économique brute des poissons produits à l lchkeul en 2015

\begin{tabular}{|lcccccc}
\hline Type de poissons & Anguilles & Loup & Muge dorée & Mulets & Autres muges & Total \\
\hline Q (kg) & 42.019 & 99 & 42.645 & 9.218 & 3.761 & 97.742 \\
\hline Prix local moyen (DT/kg) & 9 & 17 & 6 & 9 & 9 & \\
\hline Valeur (DT) & 378.171 & 1.683 & 255.870 & 82.962 & 33.849 & 752.535 \\
\hline
\end{tabular}

Source : Arrondissement Pêche Bizerte (Quantité), STL (prix) 


\begin{tabular}{lccc}
$\begin{array}{l}\text { Services } \\
\text { d'approvisionnement }\end{array}$ & $\begin{array}{c}\text { Services } \\
\text { d'approvisionnement }\end{array}$ & Valeur (DT) & Valeur (DT/ha) \\
\cline { 2 - 4 } & Olives & 8.933 & 0,7 \\
Forêts & Câpres & 942 & 0,1 \\
& Caroubes & 45 & 0,0 \\
& Bois & 1.522 & 0,1 \\
& Pâturage & 80.826 & 6,4 \\
& Nettoyage, relaxation & 25.169 & 2,0 \\
Marais & et soin au Hammam & 24.078 & 1,9 \\
Lac & Nectar & 567.927 & 45,1 \\
Total & Paturage & 440.535 & 35,0 \\
& Poissons & 1.149 .978 & 91,3
\end{tabular}

\section{Récréation}

Parmi les services culturels, la récréation est le seul service évalué pour des raisons de disponibilité d'informations. Le nombre de visiteurs du Parc national de l'Ichkeul était de 43.074 en 2015 et de 48.800 visiteurs en 2014, deux fois plus élevé qu'en 2006 (figure 6) alors que les écosystèmes du parc commençaient à se rétablir suite à la période de sécheresse. Certains visiteurs viennent pour visiter la zone extérieure du lac sans entrer dans le parc. Le nombre de visiteurs est donc plus important que celui déclaré officiellement par le conservateur du parc. Les Tunisiens forment la majorité des visiteurs (95\%), provenant principalement des villes

avoisinantes et du Grand Tunis. Le PNI offre de nombreuses attractions touristiques, notamment des sentiers aménagés pour la randonnée, un musée écologique, avec un site pour 'observation d'oiseaux et des buftes, en plus de l frequenation du hammam.

On distingue 3 saisons de visites, la haute saison (de mars à mail, avec un afflux important pendant les vacances de prinemps, la basse saison (de juin à septembre) et la moyenn saison (d'octobre à février). Deux types de visites peuvent tre distingués :

Les vistes récreatives : Selon Ben Belgacem (2013), les motifs de ces visites sont :

Figure 6 : Evolution du nombre de visiteurs au Parc National de l'Ichkeul

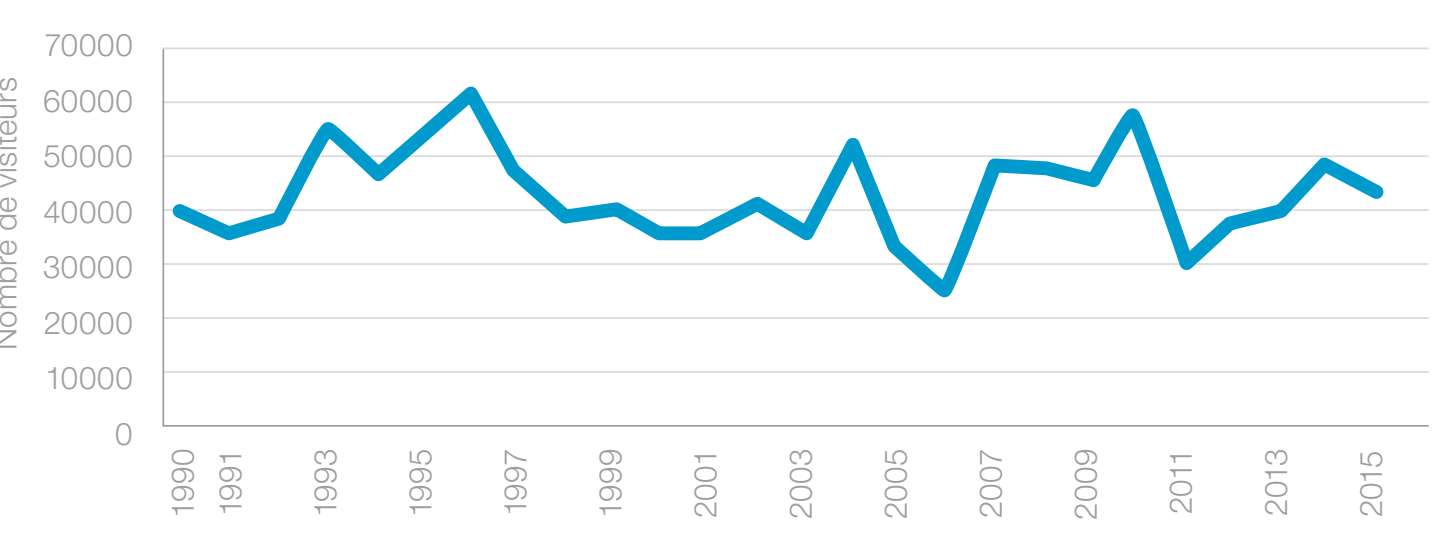

Source : Données fournies par le conservateur (Arrondissement des forêts de Bizerte)
- La découverte de la nature (40\%)

- La fréquentation du Hammam (24\%) pour les habitants locaux et les originaires des villes voisines:

La recherche d'un cadre favorable (17\% de visiteurs) pour

le pique-nique, se ressourcer et rompre avec le quotidien ou pour visiter la famille dans la région

- Etude et projet sur la diversité biologique, observation de la nature, notamment pour les ornithologues $(7 \%)$

La recherche d'un espace de liberté (6\%);

Selon une autre enquête réalisée en 2000 auprès de 600 personnes environ (Zaine, 2004), l'observation de la nature (75\%) constitue la principale motivation des visiteurs, sulvie par la promenade $(68 \%)$, le calme $(49 \%)$, le pique-nique (26\%), autres (recherche, études) (11\%).

Les visites éducatives : Le PNI accueill un nombre important de classes scolaires qui viennent pratiquer des classes nature. Selon l'enquête menée par Ben Belgacom (2013) Ies visiteurs proviennent de Tunis (35\%), Bizerte (24\%), Sousse (18\%) et d'autres localités (23\%). Les visiteurs viennent prinpalement dans le cadre de leur programme éducatif (59\%) pour la découverte (23\%) et le divertissement (18\%). En 2015, il y a eu 11 visites éducatives principalement à partir des Universités, ainsi que des écoles primaires et secondaires. De plus, il y a eu trois visites scientifiques et d'etude. Les acquis de connaissance sont relatiffs à 'eau, la biodiversité, la nature et à la géologie. Des intentions de changement de comportement suite à la visite sont indiquées : le respect de la nature et la conservation de l'équilibre de l'écosystème notamment. Les travaux de recherche: Plusieurs travaux de recherche (Mastère, Thèse de doctorat, projets de recherche) sont menes afin de developper des connaissances scientifiques sur divers aspects environnementaux.

Les visiteurs peuvent directement contribuer à l'économie du parc par le paiement de droits d'entrée, l'entrée est gratuite actuellement. En plus, ils peuvent contribuer au développement de l'économie régionale et nationale. Plusieurs activités peuvent être développées autour de l'écotourism aménagement des hammams, restauration (restauran avec de la cuisine locale, cafe, buvette, points de vente des besoins essentiels surtout leau, produits d'artisanat local, services liés au transport (calèche, chameau, etc.), services récréatifs, visites éducatives (brochures, cartes, guides), accompagnement par des quides, etc. (Ben Belgacem 2013).

'enquête est effectuée sur un échantillon de 93 personnes ors des vacances scolaires au cours des journées du dimanche 20 décembre et mardi 29 décembre 2015. Les personnes ont été choisies au hasard tout en respectant la repartition zonale observée lors du recensement de 2012 . Les enquêtes organisées dimanche et mardi ont permis de rencontrer des profils de visiteurs différents (plus de visi列 des villes voisines en milieu de semaine). Aussi, le nombre de visites observé durant ces deux jours a été très variable ntre le weekend (plus de 1000 visiteurs le 20 décembre) e milieu de la semaine (300 environ le 29 décembre 2015). Les personnes ont été rencontrées en haut des escaliers menant à l'écomusée, en face de l'écomusée, et dans parking. La quasi-majorite des visiteurs rencontrés sont des tunisiens, seulement trois sont étrangers, dont deux résidents en Tunisie.

\section{Analyse descriptive des visiteurs et de leurs visites}

Dans la partie qui suit, une analyse descriptive de l'échantillon sera entamée à travers l'analyse des différents caracères sociodémographiques des individus questionnés. Les personnes qui ont accepté de répondre à notre enquête se composent de 62\% d'hommes (souvent en présence de composé en grande majorté de person etventes en groupe 83 personnes enquettes sont accompagnées d'au mons un parsonnes enquetess sont accomnues accompagnées d'au moins un enfant).

Les effectifs qui composent notre échantillon sont répartis seon les tranches d'âge suivant le Tableau 21.La classification selon lâge révèle que la catégorie appartenant à la tranche d'âge située entre 35 et 49 ans est la plus importante. Elle représente $52,7 \%$ des participants, alors que $22,5 \%$ sont des eunes dont 'âge est situé entre 25 et 34 ans. Les personnes âgées entre 50 et 64 ans représentent 17,2\% de l'échantillon objet de notre étude. Les tranches d'âge 18-24 ans et celle de plus de 65 ans sont les moins présentes.

\section{Tableau 21 : Répartition des effectifs selon lầge}

\begin{tabular}{ccc} 
& Nombre & Pourcentage \\
\hline $\mathbf{1 8}$ à $\mathbf{2 4}$ ans & 3 & 3,2 \\
\hline $\mathbf{2 5}$ à 4 ans & 21 & 22,6 \\
\hline 35 à 49ans & 49 & 52,7 \\
\hline $\mathbf{5 0}$ à 64ans & 16 & 17,2 \\
\hline >65ans & 4 & 4,3 \\
\hline Total & 93 & 100 \\
\hline
\end{tabular}


La plupart des enquêtés (52\%) ont un niveau d'étude univer- entre 5.000 et $10.000 \mathrm{DT}, 7.8 \%$ entre 2.000 et $5.000 \mathrm{DT} / \mathrm{an}$ sitaire, alors que $41 \%$ ont un niveau d'étude secondaire, et alors que 15,5\% gagnent moins de $2.000 \mathrm{DT} / \mathrm{an}$. Seulement seulement $7 \%$ ont un niveau d'étude primaire. $3,4 \%$ de l'échantillon gagnent plus de 50.000Dt/an. Parmi Le tableau 22 illustre la répartition des individus questionnés les 93 visiteurs interrogés sur le site, $41 \%$ visitent le parc pour en fonction de leurs activités professionnelles. Leéchantillon la première fois de leur vie. Le tableau 23 présente une ré comporte 69,3\% de personnes ayant une activité professionnelle dont $47,3 \%$ sont des cadres supérieurs. $8,6 \%$ exercent leur nombre de visites au cours des douze derniers mois.

des professions libérales. De faibles pourcentages de 8,6\% En demandant aux personnes questionnées s'ils connaissent

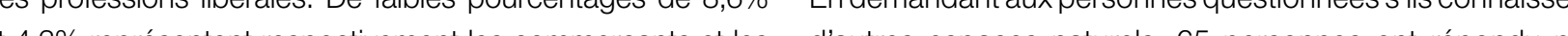

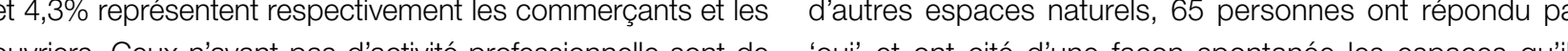

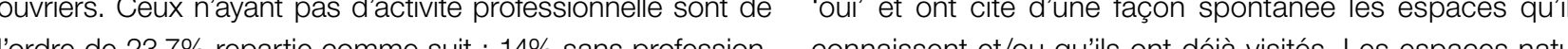
2.2\% des el 2,2\% des étudiants et 7,5\% des retraités (cf. Tableau 22). r rels cités ont eté classés en 5 catégories, à savoir; les parcs La répartition suivant les revenus annuels tels que déclaés par les personnes questionnées montre que $27,8 \%$ des Feja, Chaambi, elc.), les parcs urbains (Nahli, Sidi Bou ménages enquêtés gagnent entre 20000 et 50,000DT/an, Said, etc.), les parcs animaliers (Friguia, Belvédère, etc.) les 25.5\% gagnent entre 10.000 et $20.000 \mathrm{DT} / 2 n$. 20\% gagnent sources d'eaux thermales (Korbous, Hammam Zriba, etc.) le désert tunisien. (Tableau 24).

Tableau 22 : Répartition des effectifs selon l'activité professionnelle

\begin{tabular}{lcc} 
& Nombre & Pourcentage \\
Cadre supérieur & 44 & $47,3 \%$ \\
\hline Sans emploi & 13 & $14,0 \%$ \\
\hline Commerçant & 8 & $8,6 \%$ \\
\hline Profession Libérale & 8 & $8,6 \%$ \\
\hline Retraité & 7 & $7,5 \%$ \\
Autre & 7 & $7,5 \%$ \\
Ouvrier & 4 & $4,3 \%$ \\
Etudiant & 2 & $2,2 \%$ \\
\hline Total & 93 & $100 \%$
\end{tabular}

Tableau 23 : Nombre de visites au cours des douze derniers mois

\begin{tabular}{lcc}
\hline Le nombre de visites & Nombre & Pourcentage \\
\hline Visite du parc pour la 1èrefois de sa vie & 38 & $40,9 \%$ \\
\hline $\begin{array}{l}\text { Visite du parc la 1èrefois cette année, } \\
\text { mais l'a déjà visité auparavant }\end{array}$ & 23 & $24,7 \%$ \\
\hline Entre 2 et 8 fois cette année & 21 & $22,6 \%$ \\
\hline Entre 9 à 15 fois cette année & 7 & $7,5 \%$ \\
\hline Entre $\mathbf{1 6}$ à $\mathbf{7}$ fois cette année & 1 & $1,1 \%$ \\
\hline Entre 40 à 80 fois cette année & 3 & $3,2 \%$ \\
\hline Total & $90^{*}$ & $100 \%$
\end{tabular}

Trois personnes n'ont pas donné de réponses
Tableau 24 : Répartition des effectifs connaissant au moins un espace naturel

\begin{tabular}{ccccccc}
$\begin{array}{r}\text { Espace } \\
\text { naturel }\end{array}$ & $\begin{array}{c}\text { Source des } \\
\text { eaux thermales }\end{array}$ & $\begin{array}{c}\text { Parcs } \\
\text { Nationaux }\end{array}$ & $\begin{array}{c}\text { Parcs } \\
\text { Urbains }\end{array}$ & $\begin{array}{c}\text { Parcs } \\
\text { Animaliers }\end{array}$ & $\begin{array}{c}\text { Sud } \\
\text { Tunisien }\end{array}$ & $\begin{array}{c}\text { Autres : } \\
\text { Parcours, forêts. }\end{array}$ \\
\hline Nombre & $14 \%$ & $33 \%$ & $27 \%$ & $13 \%$ & $6 \%$ & $28 \%$ \\
\hline & 9 & 21 & 17 & 8 & 4 & 18
\end{tabular}

Certaines personnes connaissent plus d'un type de parc

Tableau 25 : Motivations de visite du parc
Espace naturel

Découverte de la nature

Récréation, calme et tranquillitè

Observation des animaux

Projets scientifiques

Hammam

\section{$1^{\text {er }}$ motif de visite}

$65 \%$

$10 \%$
$21 \%$

$45 \%$

$5 \%$

$8 \%$
La motivation de visite du parc la plus citée selon les visiteurs accéder au parc pour l'adulte et pour l'enfant (ce qui explique est la découverte de la nature, (65\% des visiteurs la classent l'écart-type assez élevé). Une analyse plus détaillée a permis comme la première raison). La deuxième étant la récréation, la classification des effectifs selon les valeurs de droit d'enle calme et la tranquillité (25\% la classent comme première trée proposés par les visiteurs (Tableau 27).

raison). On trouve ensuite l'observation des animaux (Buffles, Trois visiteurs ont exprimé qu'ils ne sont pas disposés à oiseaux) ane $4 \%$ des visteurs des droits d'entrée au parc et ont présenté une proson. D'autres raisons de visite ont eté citées telles que les testation en invoquant des raisons de refus classées comme projets scientifiques et les Hammams (Tableau 25). des « faux zéro" dans la littérature, telle que "ce n'est pas à La moitié des visiteurs passent $2 \mathrm{~h}$ ou moins dans le parc, moi de payer " seulement $25 \%$ passent plus de $3 \mathrm{~h}$ dans le parc pour effectuer des randonnées, pique-niques, etc. (Tableau 26).

Sur une échelle de 1 à $10,47 \%$ des personnes consultées $(93$ individus) sont très satisfaits de leurs visites et ont exprime un degré de satisfaction supérieur à $8.34 \%$ des personnes sont satisfaits de leurs visites et ont exprimé un degré de satisfaction entre 6 et 8 . 15\% des personnes sont moyennement satisfaites de leur visite et ont exprimé un degré $d$ satisfaction entre 4 et 6 , alors que $4 \%$ ont exprime de satisfaction inférieur à 4 . La retounner sur le site une autre fois.

Les individus qui sont favorables pour l'instauration de droit d'entrée (87 personnes, soit 93\% de l'échantillon) ont proposé un montant maximum qu'is sont disposes a payer pour

\section{Tableau 26 : Temps de visite passé dans le parc}

\begin{tabular}{|c|c|c|}
\hline Durée & Nombre & Pourcentage \\
\hline$<1 \mathrm{~h}$ & 13 & 14,3 \\
\hline 1 à $2 \mathrm{~h}$ & 35 & 38,5 \\
\hline 2 à $3 h$ & 20 & 22,0 \\
\hline 3 à $4 h$ & 9 & 9,9 \\
\hline $4-5 h$ & 10 & 11,0 \\
\hline 5 - 6 h & 4 & 4,4 \\
\hline Total & $91^{*}$ & 100 \\
\hline
\end{tabular}


Tableau 30 : Coût moyen de la visite au PNI par zone d'origine

\begin{tabular}{|ccc|}
\hline Zone & Distance Aller simple $(\mathbf{k m})$ & Coût moyen (DT/ personne) \\
\hline Tinja & 7 & 0,99 \\
\hline Mateur/Menzel Bourguiba & $13-15$ & 3,2 \\
\hline Bizerte & 38 & 7,18 \\
\hline Tunis & $75-93$ & 16,45 \\
\hline Sousse & 228 & 45,6 \\
\hline
\end{tabular}

Coût de la visite Le coût de la visite est estimé tenant à lélaboration d'une fonction de demande qui explique le Cout de la visie Le cout de la viste est estime tenat à de variables sociodémographiques.

P). Le coût de transport tient compte de la dépréciation Deux approches zonale et individuelle ont été utilisées pour l'évaluation économique.

moyennes, le coût de kilométrage d'une voiture est estimé à Approche zonale

0,4 DT/km er celu dune moto est de 0,2 DT/Km. Les couts moyens de visite obtenus sont présentés dans le tableau 30 .

$$
\mathrm{Ct}=\left(2{ }^{*} \mathrm{D}^{*} \mathrm{CM}\right) / \mathrm{P}(\mathbf{1})
$$

La majorité des enquêtés (95\% des 93 enquêtés) font l'aller-retour depuis leur résidence principale dans la même journée et un pourcentage de l'ordre de $5 \%$ font le déplacement depuis leur résidence de vacances dans la région de Bizerte, principalement à la ville de Bizerte ou Menzel Bourguiba La totalité de ces enquetés se logent dans des Bourguiba. La totalié do ces enquetes se logent dans des dans leur résidence secondaire dans la région.

La plupart des visiteurs interrogés sur le site sont venus avec leurs voitures privées $(76,6 \%)$ et ont parcouru une distance moyenne de 60,6km (soit 121,2 km en aller-retour), soitun cout dransport moyen de 12,5 DT et sont pour 4 en moyenne.

\section{Détermination de la valeur récréative du PNI}

Une fonction de régression a été établie entre le coût de la visite et le taux de visite par zone, ce dernier étant le nombre de visiteurs divisé par la population de chaque zone (figure 7). Une bonne corrélation a été obtenue tion logarithmique $\left(R^{2}=0,99\right)$

\section{$\mathrm{Ct}_{\mathrm{i}}=-4,687 \operatorname{Ln}($ Taux de visite) $-6,408$ (2)}

Ct: Le coût de transport correspondant à la zone i.

Taux de visite : ratio Nombre de visites/population de la zone A partir de cette fonction de demande, la valeur de la visite (surplus) a eté obtenue. La propension a payer (PAP) a éte calculée à partir de l'intégrale de la fonction de demande. La PAP totale est obtenue en la multipliant par la population. Soustraire le coût de visite de la propension à payer permet d'obtenir le surplus. Le coût total est calculé en multipliant le surplus a été estimé à 233.098DT/an (Tableau 31).

Etant donné que cette approche prend en compte un cout de transport unique pour chaque zone et une certaine hoL'objectif de l'analyse qui suit est la détermination de la valeur mogénéité des caractéristiques socio-économiques, l'aprecréative du Parc National Ichkeul par la méthode des coûts s proche individuelle sera donc considérée. de transport La valeur récréative peut être déterminée sulte
Figure 7 : Fonction de demande récréative du Parc National lchkeul

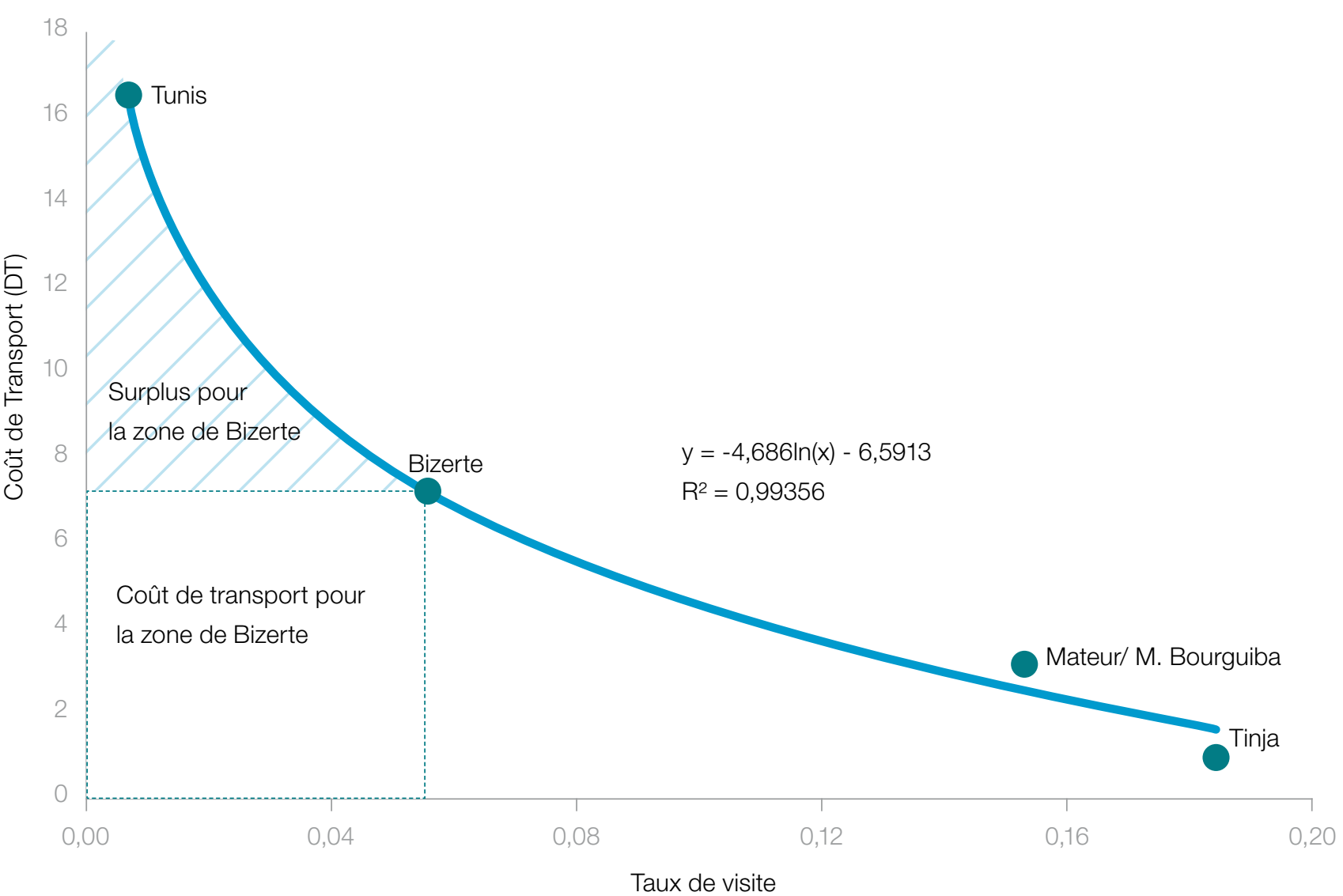

Tableau 31 : Propension à payer, coût de visite et surplus

\begin{tabular}{lcccccc}
$\mathbf{2 0 1 2}$ & $\begin{array}{c}\text { Nombre de } \\
\text { visiteurs }\end{array}$ & Population & $\begin{array}{c}\text { Taux de visite } \\
\text { (Nombre } \\
\text { de visites/ } \\
\text { habitant) }\end{array}$ & $\begin{array}{c}\text { Valeur du site } \\
\text { récréatif }\end{array}$ & $\begin{array}{c}\text { Pap moyenne } \\
\text { (DT/visite) }\end{array}$ & Surplus (DT) \\
\hline Tinja & 2.185 & 23.455 & 0,18 & 0,99 & 6,33 & 23.013 \\
\hline $\begin{array}{l}\text { Mateur/ } \\
\text { Menzel B. }\end{array}$ & 10.494 & 135.871 & 0,15 & 3,2 & 7,21 & 82.966 \\
\hline $\begin{array}{l}\text { Bizerte } \\
\text { Tunis }\end{array}$ & 4.821 & 167.759 & 0,06 & 7,18 & 11,84 & 44.331 \\
\hline Total & 8.865 & 2.264 .183 & 0,01 & 16,45 & 21,18 & 82.788 \\
\hline \multicolumn{7}{c}{ Valeur du site récréatif } \\
\end{tabular}




\section{Approche individuelle}

Le modèle de Poisson est le modèle de base utilisé afin d'estimer la fonction de demande et de determiner l'équation dècrivant l'introduction des variables explicatives. Pour estimer la fonction de base, on suppose que les visiteurs manifestent une demande pour le site récréatif en fonction du coût de transport (Ct) qu'ils payent pour s'y rendre. La demande est exprimée par le nombre de visites $\left(N_{j}\right)$, en supposant que loffre des biens et des services reste constante.

Pour un visiteur i, la demande est exprimée par la fonction

$$
N_{i}=f\left(C t, X_{i}\right)
$$

Avec $X_{i}$ : vecteur de caractéristiques du visiteur

Par ailleurs, sachant que la seule variable explicative utilisée dans cette étude est "le revenu", la fonction de demand d'un individu i s'écrit ainsi :

$$
N_{i}=\beta_{0}+\beta_{1}{ }^{*} C_{i}+\beta_{1}{ }^{*} R_{i}+\beta_{2}{ }^{*} M_{i}(4)
$$

Avec :

$N_{1}$ : Le nombre de visite effectué par l'individu i au cours des 12 derniers mois.

$\mathrm{Ct}_{1}$ : Le coût de transport supporté par l'individu i.

$R_{i}$ : Le revenu moyen par ménage de l'individu i.

Mi : Statut familial (Si l'individu i est marié $\mathrm{Mi}=1$, sinon $\mathrm{Mi}=0$ ). $i$ : Indice d'observation

$\beta_{0}:$ La constante.

$\beta_{1}, \beta_{2}$ : Les coefficients estimés pour les variables exogènes.

Une fois que le modèle est estimé, les paramètres sont utilisés pour calculer le surplus pour chaque personne de l'échantion. Ce dernier mesure le bien-être des visiteurs lie a l'existence du site, autrement dit : 'la valeur d'usage recreatif du

site'. On peut ensuite calculer une valeur moyenne, cette estimation est ensuite extrapolée à la population générale.

L'estimation de l'équation précédente par le logiciel STATA On a fourni les resultats figurant au tableau 32.

(146,95e aussi que la valeur du ratio de vraisemblance $R(146,95)$ est supérieure à la valeur statistique du $\times 2$ tabulaire à 2 degrés de liberté $(x 2(2,005)=5,991)$. Donc modèle est significatif dans sa globalité.

Ainsi l'équation de demande récréative du 'PNI s'écrit sous

$N_{i}=1,046198-0,07711^{*} C t_{i}-2,3 E-05^{*} R_{i}+1,270468^{*} M_{i}$ (5)

Les résultats obtenus montrent que les variables : coût de transport et revenu ont un effet significatif au seuil avec un impact négatif, alors que la situation familiale a un impact positif sur la demande récréative.

A partir de cette équation, on détermine le surplus du consommateur qui mesure le bien-être des visiteurs lié à l'existence du site, autrement dit : 'la valeur d'usage récréatif du site'. On distingue deux types de surplus du consommateur : le surplus mateur par visite $\mathrm{SC}_{\mathrm{i}}^{\mathrm{v}}$

Sachant que pour le modèle de comptage utilisé 'Modèle de Poisson', 'esperance est calculée par l'expression qui suit :

\section{$E[N / x]=\lambda i=e\left(x^{*} B i\right)(6)$}

Le surplus du consommateur par an est ainsi calculé comm "intégrale du paramètre de distribution par la formule suivante :

$$
\mathrm{SC}_{i}^{\top}=\int_{1}^{n} \lambda^{i *} \mathrm{dct}=\int_{1}^{n} \mathrm{e}^{\mathrm{Ni*} d \mathrm{dct}}
$$

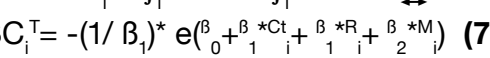

\section{Tableau 32 : Résultats d'estimation du modèle}

$\begin{array}{lccc} & \text { Coef. } & \text { Std. } & \text { P-Value } \\ \text { Ct } & -0,07711 & 0,010516 & 0.000^{\star \star \star} \\ \mathbf{R} & -2,3 \mathrm{E}-05 & 4,51 \mathrm{E}-06 & 0.000^{\star \star \star} \\ \mathbf{M} & 1,270468 & 0,295066 & 0.000^{\star \star \star} \\ \text { Constante } & 1,046198 & 0,296639 & 0.000^{\star \star \star}\end{array}$

notre étude est calculé par :

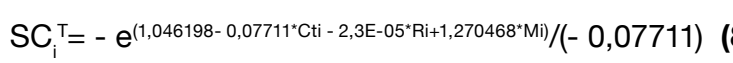

a valeur récréative du 'Parc National de l'Ichkeul' par individu et par visite, suivant l'équation (5), est de 12,01 DT. Puisque le nombre de visites pour l'année 2015 a été estime a 46.520 visiteurs, la valeur récréative totale du parc serait de l'ordre de $\mathbf{5 5 8 . 7 0 4}$ DT.

4.2 Valeurs économiques des bénéfices indirects fournis par le PNI Ce paragraphe présente une évaluation des cinq bénéfices indirects identifiés.

PRÉVENTION DE L'ÉROSION ET DE LA SEEDIMENTATION DU LAC GRÁCE A LA COUVERTURE FORESTIÈRE

La prévention de l'érosion hydrique dans la montagne a une incidence directe sur le maintien de la fertilité du sol et sur la sédimentation au niveau des marais et du lac et par conséquent sur la biodiversité du parc. Autrement, le cumul des sédiments au fond du lac et dans les marais accentue le niveau de risque de l'inondation, au moment des crues, des infrastructures de la ville de Mateur et des terres agricoles de la plaine.

est important de mentionner qu'il y a eu un défrichemen des terres forestières au début des années soixante et qu'elles ont été transformées en exploitation agricole pour la production céréalière et fourragère. Ces terres ont été dans un premier temps incluses dans des coopératives agricoles de production durant la période de collectivisation puis en seconde période cédées au domaine de l'Etat avant d'être restituées à la DGF qui a interdit son exploitation agricole (CRDA Bizerte 2006). Cette reconversion en forêt dénote Olonté de l'Etat d'éviter les dommages causées par tation agricole en termes d'érosion et de sédimentation. aleur totale des bénéfices directs

Au total, la valeur économique des bénéfices directs est estimé à 1,708 Milions DT en 2015, composee de services d'approvisionnement (1,150 Million DT) et de services culturels (0,558 Million DT). En plus de cette valeur, le PNI fournit des bénéfices indirects qui sont évalués dans la section suivante. 
PROTECTION CONTRE LES INONDATIONS

Le lac et les marais servent de bassin de rétention des eaux de pluie et de déversement des eaux pluviales de toute la région de Mateur et Ghezala. Autrement, la ville de Mateur et es terres agricoles subissent des inondations.

La surface des terres agricoles (cultures céréalières et fourragères) dans la périphérie du parc est de $16.407 \mathrm{ha}$. Les plaines situées en amont des marais (aval de Joumine, Ghezala et Maleh), d'une superficie de 5.500 ha, sont munis d'un système de drainage ${ }^{17}$ (Aouididi 1996). Ces terres sont utilisées principalement en cultures céréalières. Des pertes de rendement agricole ont été observées à cause de l'engorgement des terres. Le pompage vers le lac permet une amélioration des rendements des cultures menées en sec th une meilleure plus-value des cultures environnantes, no-

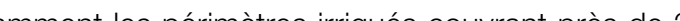
(Erija, 2006).

Par ailleurs, ce système a permis de déverser un débit annuel moyen de 3,4 Million de $\mathrm{m}^{3}$ au cours de la période (1995-2006) (Frija, 2006) et de 2,4 Million de $\mathrm{m}^{3} \mathrm{par}$ an (20122014) (données fournies par le CRDA, 2015). Ce déversement d'eau représente une situation gagnant-gagnant pour 'exploitation agricole des terres périphériques et pour la préservation de la biodiversité du PNI.
Toutefois, si le niveau d'eau augmente au niveau des marais et du lac par rapport aux terres agricoles, le pompage eeut plus être effectué et des inondations seraient alors observées au niveau des terres agricoles. En effet, selon 'OTD, les surfaces inondées atteignent 1000 ha, soit 18\% des terres agricoles concernées par le système de drainage en moyenne sur la période (2012-2015) (cf. figure 8).

La valeur relative à la protection des terres contre les inondations est estimée sur la base des côts des dommages évités (pertes de production) pour les terres non inndées. Etant donné que les inondations sont des érìnements ire

A cause des fortes crues en 2012, la surface inondée a été estimée à 2.000 ha (Communication avec un responsable au CTV Mateur). Pour ces terres, la perte de production étal totale sur une superficie de 1000 ha et seulement la moitte de la production pour le reste (CTV Mateur).

Selon la même source, en plus de cette perte de production partielle evitee, la surface totalement protégee par pompage de l'eau dans le lac a été donc estimée à 3.500 ha en 2012, le tiers de cette surface (1.166 ha) en 2013 et valeur de la production de la culture du ble par l'OTD est estimée à 1.408 DT/ha (Communication avec guliers, une moyenne sur trois ans est considérée. nulle en 2014, soit 1.555 ha/an en moyenne durant la période

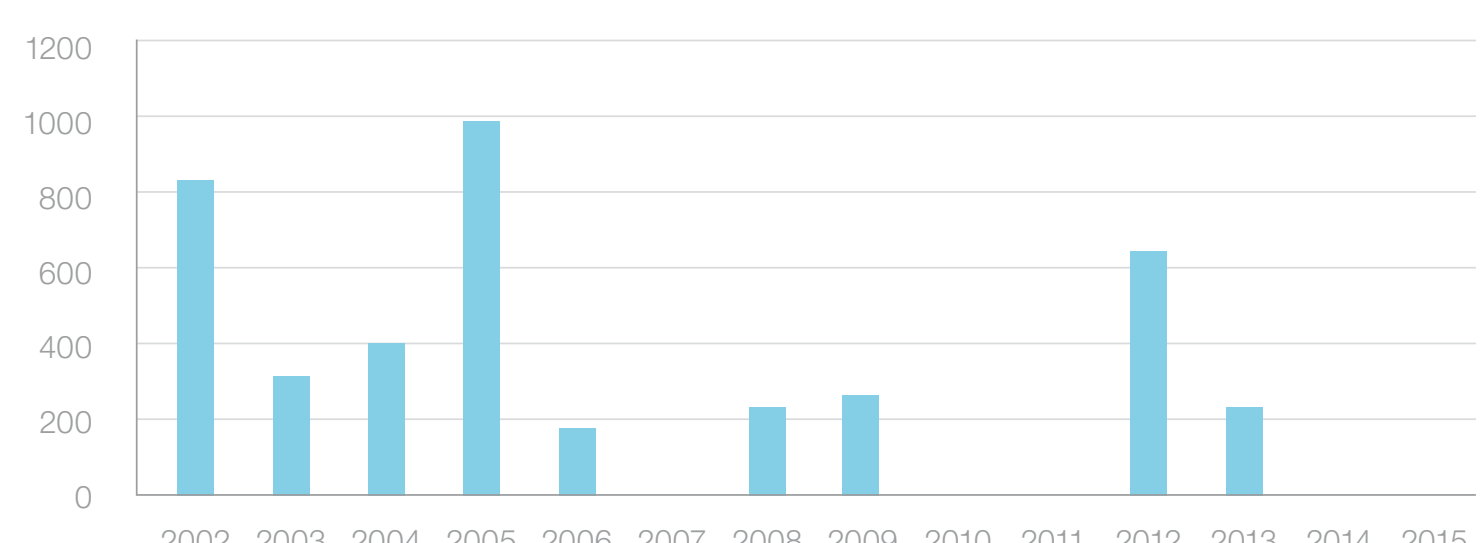

I'OTD et un agriculteur), on soustrait de cette valeur bénéfice relatif au pacage (70-80 DT/ha), en effet, certains agriculteurs louent leurs terres pour le pacage en cas d'inondations.

La valeur brute relative à la protection contre les inondations est ainsi estimée à 2,37million DT. On soustrait le coût de pompage vers le lac de cette valeur (250.000 DT/ an avec $0,105 \mathrm{DT} / \mathrm{m}^{3}$ Communication CRDA) pour obtenir une valour nette de $\mathbf{2 , 1 2}$ Million DT.

Aussi, le lac joue un rôle de contrôle de crues exceptionnelles. En effet, les lâchers d'eau dans le lac à partir des barrages sont très élevées lorsque le volume de régulation des barrages est dépassé. Pour le barrage Joumine par exemple, les lâchers d'eau (déversement, dévasement) étaient de $118 \mathrm{M}$ $\mathrm{m}^{3}$ en 2003, $112 \mathrm{M} \mathrm{m}^{3}$ en 2005 et $87 \mathrm{M} \mathrm{m}^{3}$ en 2012 (DGBTH).

\section{RÉTENTION DE SÉDIMENTS PAR LE LAC}

Le lac joue un rôle de rétention de sédiments qui proviennen des oueds et des barrages. En effet, des opérations de dévasement (lâchers des eaux boueuses) des barrages environnants sont régulièrement effectués en cas de fortes pluies, ceci entraîne des capacités en eau supplémentaires des réservoirs d'eau de ces barrages au détriment de la sédimentation du lac.

Létude de la sédimentation du lac qui compare les bathymetres de 1994 et 2004 (Ouerghi, 2006 cité par MARH, 2006 ) a permis de déduire que le lac a reçu durant ces 10 années environ $10 \mathrm{Mm}^{3}$,soit 1 Million de $\mathrm{m}^{3}$ par an, qui s'ajoute aux capacités en eaux des barrages ${ }^{18}$.

L'évaluation monétaire se base sur le coût de remplacement de la capacité de stockage à travers la construction de nouveaux barrages. Ce coût est estimé de 0,9DT/ $\mathrm{m}^{3}$ pour le barrage Melah-Bizerte, situé dans le bassin versant du lac Ichkeul (Données fournies par DGBTH). Ainsi, la fonction de réduction de l'envasement des barrages est estimée à 0,9 millions DT
En contrepartie, le niveau des sédiments dans le lac augmente d'une moyenne de 12cmen moyenne depuis 1994, ce qui explique en partie l'élévation très importante des niveaux d'eau dans le lac durant l'année 2004/2005 (2,5 m NGT selon le apport scientifique) (DGF 2006). La sédimentation à l'amon de l'écluse a été très importante. Pour cela, des travaux de dragage au niveau de lécluse sont souvent réalisés par la Société STL, pour un coût annuel de 120.000 DT ${ }^{19}$. Ce coût est soustrait du bénéfice indiqué ci-dessus pour obtenir un bénefice lié à la rétention de sédiments estime à 780.000 DT/an.

\section{RECHARGE DE LA NAPPE}

e lac Ichkeul est l'exutoire d'un système aquifère dont profondeur dépasse $150 \mathrm{~m}$, contribuant à l'alimentation de mées à $12,6 \mathrm{M} \mathrm{m}^{3}$ (données fournies par CRDA Bizerte).

Un volume de 6,4 $\mathrm{M} \mathrm{m}^{3}$ est exploité en 2014 dont 5,3 $\mathrm{M}$ $\mathrm{m}^{3}$ pour l'eau potable (SONEDE) et $1,1 \mathrm{M} \mathrm{m}^{3}$ pour l'usage agricole ${ }^{20}$. On suppose que seulement la moitié des apports proviennent du lac $\left(3,2 \mathrm{M} \mathrm{m}^{3}\right)$, le reste provient des barrages périphériques, ayant un volume d'eau contrôlé de $220 \mathrm{M} \mathrm{m}^{3}$ (Frija 2006).

La valeur de l'eau pour l'usage agricole a été évaluée en suipond à la différence de bénéfice net entre la production des cultures irriguées du blé dur en 2014 (2137 DT/ha) et celle en pluvial (1408 DT/ha) et tous les autres facteurs restant constant (Communication avec Mr. Hammami, et OTD), un bénéfice additionnel de $729 \mathrm{DT} / \mathrm{ha}$ a été retenu. En consi-

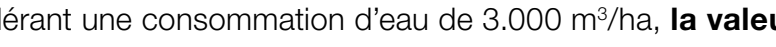
conomique de l'eau d'irrigation est de $0,24 \mathrm{DT} / \mathrm{m}$ soit un bénéfice de 132.000 DT

La valeur des bénéfices relatifs à l'eau potable est estimée de la façon suivante, en utilisant les données fournies par la SONEDE:

$B:=Q(P-C p-C t)$

$Q:$ Quantité d'eau pompée.

P. Prix de vente moyen de l'eau par la SONEDE aux consommateurs $\left(0,7 \mathrm{DT} / \mathrm{m}^{3}\right.$ en 2014). vant la méthode de changement de production. Elle corres-

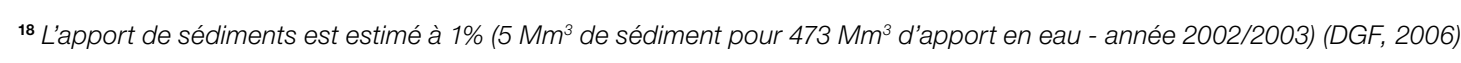
${ }^{19}$ Selon le responsable de STL, ce coût n'est pas suffisant, le coût de dragage serait de 600.000 DT pour enlever tous les sédiments au niveau

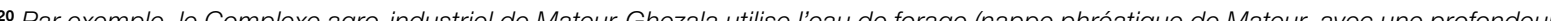
de 68 m notamment pour l'abreuvement du cheptel. 
$\mathrm{Cp}$ : Cout de pompage du forage de Mateur $\left(0,05 \mathrm{DT} / \mathrm{m}^{3}\right)$. $\mathrm{Ct}$ : Cout de transfert $(40 \mathrm{~km}$ de canaux de Mateur à Bizerte, durée de vie : 30 ans, taux d'escompte : 2\%) $\left(0,15 \mathrm{DT} / \mathrm{m}^{3}\right)$. leur des bénéfices directs (cf. Tableau 33). Les prinLa valeur économique de ce bénéfice est estimée à $1,32 \mathrm{M}$ cipaux bénéfices indirects sont ceux relatifs à la proDT en 2014.

Le bénéfice total relatif de la recharge de la nappe est ainsi estimé à 1,457 M DT en 2014.

\section{Tableau 33 : Valeur des bénéfices indirects en 2015}

\begin{tabular}{lccc} 
& Bénéfices & Valeur (DT) & DT/ha \\
\cline { 2 - 4 } Forêts & Prévention de l'érosion & 142.297 & 11,3 \\
& séquestration du carbone & 62.526 & 5,0 \\
Marais et lac & Protection contre les inondations & 2.119 .983 & 168,3 \\
Lac & Rétention des sédiments & 780.000 & 61,9 \\
Total & Recharge de la nappe phréatique & 1.457 .000 & 115,6 \\
& & 4.561 .806 & 381,9
\end{tabular}

tection contre les inondations et à la recharge de la nappe phréatique par le lac et les marais.

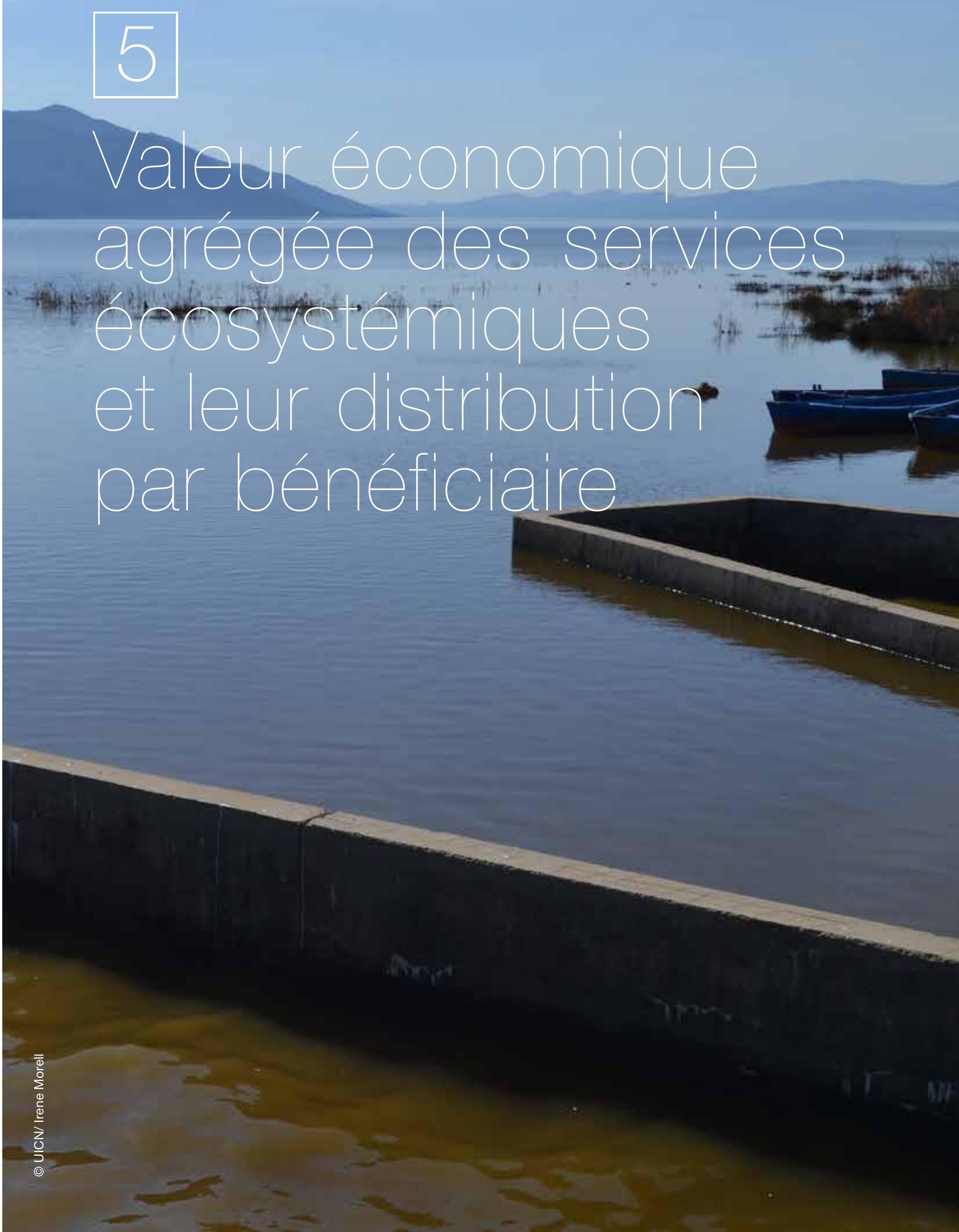


5.1 Valeur économique agrégée

La valeur économique agrégée des services écosysté- mentionnés faute d'information, on peut citer la prévention miques est estimée à 6,27 millions DT en 2015. Les béné- de la salinisation de la nappe phréatique et l'intrusion marine, fices economiques importants du parc sont la protection dont l'existence reste a vérifier.

contre les inondations (33,8\% de la valeur totale), la recharge La valeur des services écosystémiques est estimée à 497,7 de la nappe phréatique (23,2\%), la rétention des sédiments DT/ha en 2015. Elle varie selon le sous-écosystème : (i) c'est $(12,4 \%)$, le pâturage $(10,4 \%)$, la récréation $(8,9 \%$ ) et la pêche (7\%) (cf. Tableau 34). La valeur des services de régulation, ou les bénéfices d'usage indirect, constituent la majorité notamment aux multiples services de régulation, (II) vient endes bénéfices $(73 \%)$, alors que la valeur des bénéfices sur sulte les marals, gacee aux services de régulation et dupa(309,9 DT/ha),(iii) puis les forêts (298,4 DT/ha) grâce la prévention de la sédimentation et le pâturage notamment. mentionner que la valeur du service de protection contre les C'est la population tunisienne en général et la population inondations correspond à la moyenne annuelle au cours de de la région de Mateur en particulier, qui profite de la plus Tondations correspond a la moyen a pre la periode (2012-2014), cette valour pout atre tres elevee en grande part (81\%) de la valur economique des services année pluvieuse (Comme celle de 2012), mais aussi, elle peut foumis parle pare a travers les servces relatis à la régulation être nulle en annee sèche (comme celle de 2014). Toutefois, de leau (Figure 7). Malgré la faible part profftant à la populalaugmentation de la frequence des évènements extrêmes tion locale, la valeur du béneffice (pâturage notamment) par prévisibles avec les changements climatiques induirait une ménage est assez élevée : soit 3.089 DT par ménage par an augmentation de la valeur de ce bénéfice, et démontre le rôle pour la population à lintérieur du parc et 2043 DT par médu parc Ichkeul dans l'atténuation des effets du changement nage par an pour la population à l'extérieur du parc. climatique. D'autres services écosystémiques n'ont pas ête

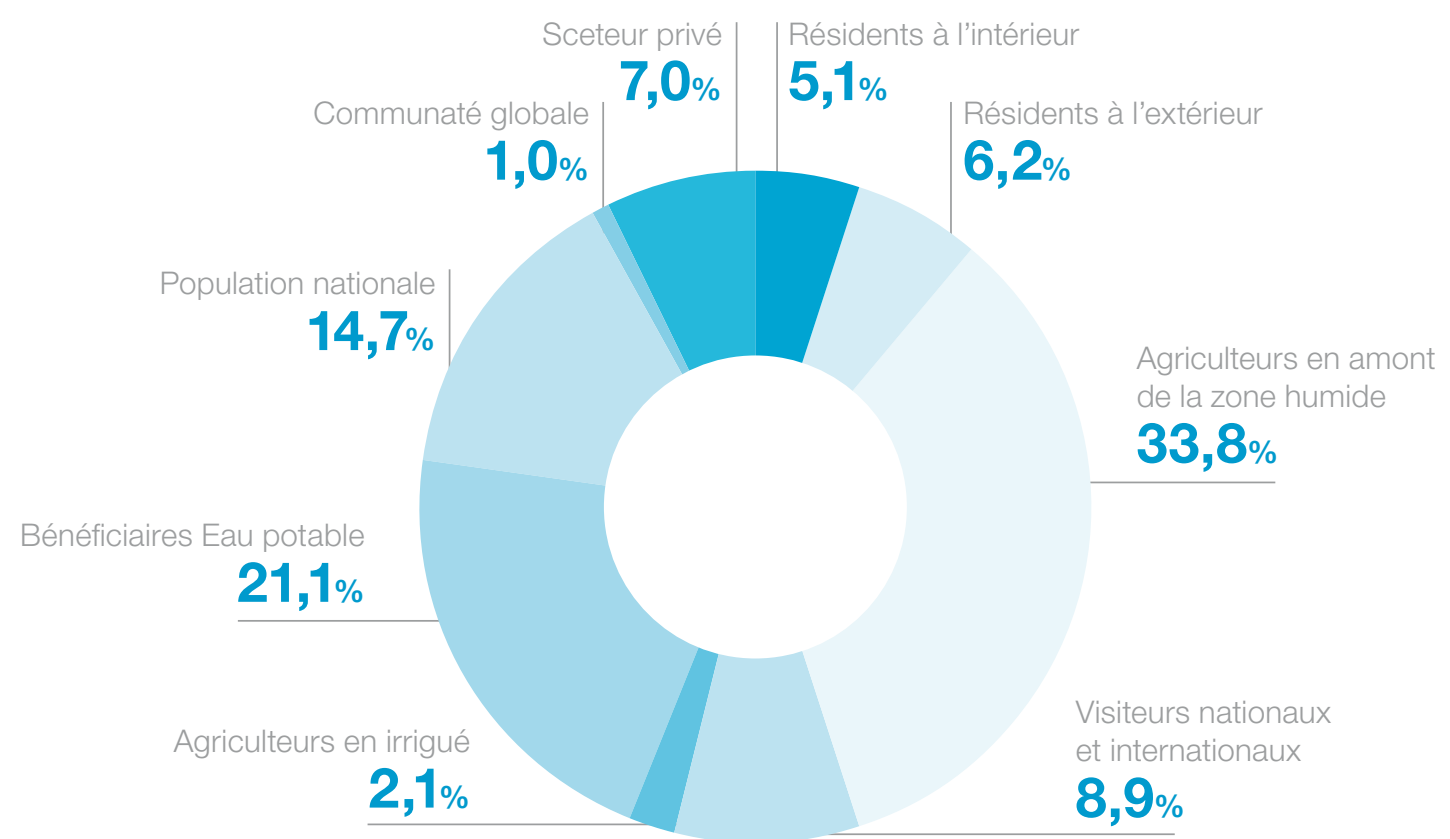

Tableau 34 : Valeurs des services écosystémiques fournis par le Parc National de l'I'chkeul (en DT) - Année 2015

\begin{tabular}{|c|c|c|c|c|c|c|c|c|c|c|}
\hline & & Q & Unité & Prix & Valeur nette & $\%$ & Valeur nette/ha parc & Valeur nette/ha forêt & Valeur nette/ha lac & Valeur nette/ha marais \\
\hline \multirow{8}{*}{ Forêts } & Service d'approvisionnement & & & & 1149978 & $18,3 \%$ & 91,3 & 103,8 & 51,8 & 207,5 \\
\hline & Olives & 14180 & $\mathrm{~kg}$ & $0,1 \%$ & 8933 & $0,1 \%$ & 0,7 & 6,6 & & \\
\hline & câpres & 349 & $\mathrm{~kg}$ & $0,0 \%$ & 942 & $0,0 \%$ & 0,1 & 0,7 & & \\
\hline & caroubes & 33 & $\mathrm{~kg}$ & $0,0 \%$ & 45 & $0,0 \%$ & 0,0 & 0,0 & & \\
\hline & Bois & 169 & $\mathrm{~m}^{3}$ & $0,0 \%$ & 1522 & $0,0 \%$ & 0,1 & 1,1 & & \\
\hline & Pâturage & 195 & 1000 UF & $1,3 \%$ & 80826 & $1,3 \%$ & 6,4 & 59,3 & & \\
\hline & Nettoyage, relaxation et soin- Hammam & 16779 & personne & 1,5 & 25169 & $0,4 \%$ & 2,0 & 18,5 & & \\
\hline & Nectar & 1331 & $\mathrm{~kg}$ & 30 & 24078 & $0,4 \%$ & 1,9 & 17,7 & & \\
\hline Marais & Pâturage & 1368 & $1000 \mathrm{UF}$ & 500 & 567927 & $9,1 \%$ & 45,1 & & & 207,5 \\
\hline Lac & poissons et autres ressources aquatiques & 98 & $\mathrm{t}$ & & 440535 & $7,0 \%$ & 35,0 & & 51,8 & \\
\hline \multirow{3}{*}{$\mathrm{PNI}$} & Services culturels & & & & 558704 & $8,9 \%$ & 44,3 & 44,3 & 44,3 & 44,3 \\
\hline & Récréation, loisirs & 46520 & visiteurs & 12,01 & 558704 & $8,9 \%$ & 33,8 & 33,8 & 33,8 & 33,8 \\
\hline & Services de régulation & & & & 4561806 & $72,8 \%$ & 381,9 & 150,3 & 429,5 & 258,1 \\
\hline \multirow{2}{*}{ Forêts } & Prévention de l'érosion & 1363 & ha & 104,4 & 142297 & $2,3 \%$ & 11,3 & 104,4 & & \\
\hline & séquestration du carbone & 1226 & $\mathrm{tCO}_{2}$ & 51 & 62526 & $1,0 \%$ & 5,0 & 45,9 & & \\
\hline \multirow{2}{*}{ Marais et lac } & Protection contre les inondations & 2000 & ha & $666-1333$ & 2119983 & $33,8 \%$ & 168,3 & 0,0 & 188,7 & 188,7 \\
\hline & Rétention des sédiments & 1000000 & & 0,90 & 780000 & $12,4 \%$ & 61,9 & & 69,4 & 69,4 \\
\hline Lac & Recharge de la nappe phréatique & 5700000 & $\mathrm{~m} 3$ & 0,24 & 1457000 & $23,2 \%$ & 115,6 & & 171,4 & \\
\hline
\end{tabular}


5.2 Comparaison avec les études antérieures sur le Parc Ichkeul

L'écosystème Ichkeul a fait l'objet de plusieurs évaluations - Ferchiou (2011) a effectué une évaluation de : (1) La valeur économiques auparavant sur des aspects particuliers :

- Thomas D. et al. (1991). ont effectué une évaluation des valeurs d'usage et de non usage de la conservation du PNI. Cette évaluation de l'écosystème, la plus complète, est parmi les premières à l'échelle nationale et internationale. Elle a évalué : (1) la valeur de la production animale attribuée au marais à 106.000 DT en 1988, (2) la valeur nette de la production de poissons à 483.000 DT en 1988, (3) la valeur attribuée au tourisme à 23.000 DT en 1988, (4) la valeur relative au traitement des eaux usées à 156.000 DT (5) la valeur attribuée à la recharge de la nappe de Mateur à 340.000 DT : soit une va a cur de lo nappe de Mateur

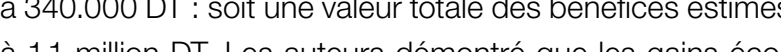
a 1,1 millon DT. Les auteurs demontre que les gains economiques de prendre des mesures de prévention de la dégradation de la zone humide, notamment des lâchers d'eau pour le maintien des marais, seraient supérieures aux bénéfices économiques de l'utilisation d'eau pour l'irrigation, et contribueraient a repondre aux accords internationaux sur la conservation du parc.

- Aouididi S. (1996) a effectué une évaluation économique de trois services écosystémiques : (1) La valeur des poissons évaluée à 828600 DT/an en utilisant le prix de marché (6 DT $/ \mathrm{kg}$ ) ; (2) La valeur récréative estimée à 241200 DT au total en utilisant la méthode de coût de transport (11,7 DT/ visite (international) et 3,9 DT/ visite (national)) ; (3) La perte de production liée à la salinité estimée à 15565 DT (solt $1,9 \%$ de la valeur initiale) en se basant sur la baisse de la valeur des poissons entre 1982-1992.

- Banque Mondiale (2007). Le coût de dégradation de la zone humide a été estimé à 104000 DT/an en utilisant la méthode de coût de remplacement (cout
dragage et de réhabilitation des marais). récréative (2,5 DT par visiteur) en effectuant une enquête sur la disposition à payer d'un droit d'ontrée auprès de 82 visiteurs en face à face sur site en 2011, (2) La valeur rethive à prése à face sur sle en 2011, (2) La valeur repar visiteur) en effectunt une enquete sur la dispos, DT payer auprès des mêmes visiteurs.

Lamparaison des résultats de cette étude avec les études (Tableau 35) montre que :

- La valeur des poissons a diminué dû au fait de la baisse de la quantité produite (98 t en 2014 contre 138 t en 1995) et la faible augmentation du prix relativement au taux d'inflation.

- La valeur du pâturage a augmenté du fait de l'augmentation des prix des céréales notamment.

- La valeur récréative a aussi diminué à cause de la baisse du nombre de visiteurs au cours des dernières années.

- La valeur de la recharge de la nappe a augmenté grâce à l'amélioration de la valeur de productivité de l'eau.

- La valeur relative aux traitements des eaux usées est nulle actuellement car l'Etat a mis en place (après 1988) un système de traitement des eaux usées et il n'y a plus de deversement des eaux usées dans le lac.

- La valeur économique estimée par cette étude est presque le double que celle obtenue par Thomas et al. (1991).

En effet, malgré que ces derniers ont estimé certains services de régulation, d'autres services n'ont pas été évalués : les valeurs de la protection contre les inondations, la rétention des sédiments, la séquestration de carbone, les produits forestiers, etc. Cette étude les a estimées pour la première fois.
Tableau 35 : Comparaison des valeurs de certains services écosystémiques (Valeur en 1000 DT - prix 2015)

\begin{tabular}{lccc} 
& Thomas (1991) & Aouididi(1996) & Résultats de cette étude \\
\hline Année de l'évaluation & 1988 & 1995 & 2015 \\
\hline Pâturage & 340,5 & & 648,8 \\
\hline Poissons & 1537,9 & 1678,0 & 440,5 \\
\hline Récréation & 74,0 & 488,5 & 558,7 \\
\hline Traitement des eaux usées & 497,4 & & 0 \\
\hline Recharge de la nappe & 1084,1 & & 1457,0 \\
Valeur totale estimée & 3535,3 & & 6270,5
\end{tabular}

5.3 Comparaison internationale des valeurs monétaires des services fournis par les zones humides intérieures végétalisées

Une étude basée sur 89 études de cas a montré que la valeur médiane des services varie selon le type de zone humide de 120 à 374US \$/ha/an en 2000 (cf. Tableau 36) et que la valeur moyenne est de 54 \$ha les services foumis par les zones humides de rétention de sédiments suivis par les zones humides boićes pour la foumiture de leau ont les valeurs les pus éléés (\$374 Tes valus les plus eloveos (\$374 /ha/ an et \$206/ha/an respectivement) (Schuyt and Brander, 2004). La presente étude estime la valeur moyenne pour le PNI à 256 US \$/ha en 2015, valeur superieure à la moyenne mondiale. Elle es plus élevée que celle des marais saumâtres (165 US \$/ha en 2000). Brander et Schuyt (2010) ont utilisé une approche de transfert de valeurs pour l'estimation de la valeur des zones humides dans le monde. En utilisant une analyse de régression : une fonction de la valeur des zones humides a éte déterminée utilisant un nombre de variables tels que le type de zone humide, niveau de vie par habitant, la dens la population et la taille de la zone humide.

Une autre étude élaborée par de Groot (2012) et reprise par TEEB (2010) sur les zones humides a présenté des valeurs

seule information disponible correspond au parc national de Doñana, Espagne (170.000 ha), il a été indiqué que la production directe (agriculture, élevage, pêche, ressources marines) est de $1990 € /$ ha, les services de régulation (protection contre les espèces invasives, etc.) ont une valeur de $154 €$ /ha et la valeur des services culturels est de $1212 € /$ ha Weber, J.-L. et al. (2008) citée in Agence de l'eau Adour-Garonne, 2009)

Ces valeurs sont assez élevées par rapport à celles obtenues dans la présente étude. Toutefois, il a été indiqué que ces valeurs ne peuvent être prises qu'à titre indicatif car il s'agit d'une compilation de valeurs à partir d'études préexisantes dont les hypothèses ne sont pas précisées.

Plus spécifiquement, le tableau 38 compare la valeur obtenue sur les services récréatifs (1̇̀re colonne) avec les valeurs obtenues dans deux parcs (Parc naturel de l'Albufera, Parc naturel de las Tablas) en Espagne. Les valeurs sont dans le même orde de grandeur surtout si l'on tient compte de parité du pouvoir d'achat (PPA) (dernière ligne).

moyennes par ha très élevées sans aucune autre précision sur le pays et la surface des zones humides (Tableau 37). nous a paru intéressant de comparer les valeurs obtenues avec les zones humides similares concerńes par la migration des oiseaux. 
Tableau 36 : Valeurs économiques des services écosystémiques selon le type de zones humides (US \$/ha/an, 2000)

6

Vasières / zones Vasières / zones

Marécages, plaines

Valeur

$$
374
$$
terres boisées

Marais d'eau terres boisees 105 rat 145

Mangroves Source: Schuyt, K., And Brander, L. 2004

Tableau 37 : Comparaison des valeurs des services écosystémiques obtenues avec les estimations internationales au niveau des zones humides végétatives intérieures

\begin{tabular}{|c|c|c|c|}
\hline Services & $\begin{array}{l}\text { La présente étude } \\
\text { (\$/ha/an) }\end{array}$ & $\begin{array}{c}\text { Nombre d'estimation } \\
\text { utilisé }\end{array}$ & $\begin{array}{l}\text { Valeur moyenne. } \\
\text { (\$/ha/an) - } 2007\end{array}$ \\
\hline $\begin{array}{l}\text { Services } \\
\text { d'approvisionnement }\end{array}$ & 47 & 34 & 1659 \\
\hline Services culturels & 23 & 13 & 648 \\
\hline Services de régulation & 185 & 30 & 4203 \\
\hline
\end{tabular}

Tableau 38 : Comparaison des valeurs des services récréatif obtenues avec les
estimations internationales au niveau des zones humides végétatives interieures

\begin{tabular}{|c|c|c|c|c|}
\hline Site & PNI - Tunisie & \multicolumn{2}{|c|}{ Parc naturel de l'Albufera } & $\begin{array}{l}\text { Parc naturel de las } \\
\text { Tablas - Espagne }\end{array}$ \\
\hline Méthode & MCT & MCT & MEC & MEC \\
\hline $\begin{array}{l}\text { Valeur obtenue } \\
€ / \text { personne }\end{array}$ & 5,53 & 16,70 & 3,55 & 5,67 \\
\hline $\begin{array}{l}\text { Valeur obtenue } \\
\text { \$ PPA/personne }\end{array}$ & 18,20 & 24,56 & 5,15 & 8,33 \\
\hline Références & La présente étude & Del S & uarez & Judez L., et al. (2002) \\
\hline
\end{tabular}

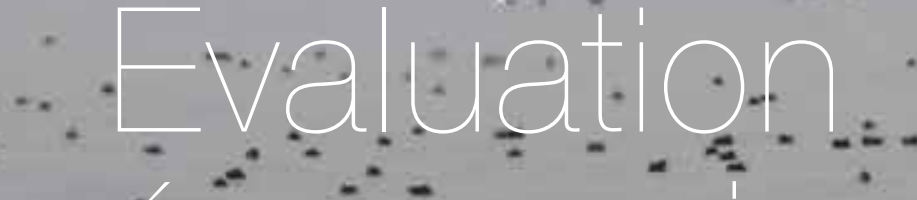

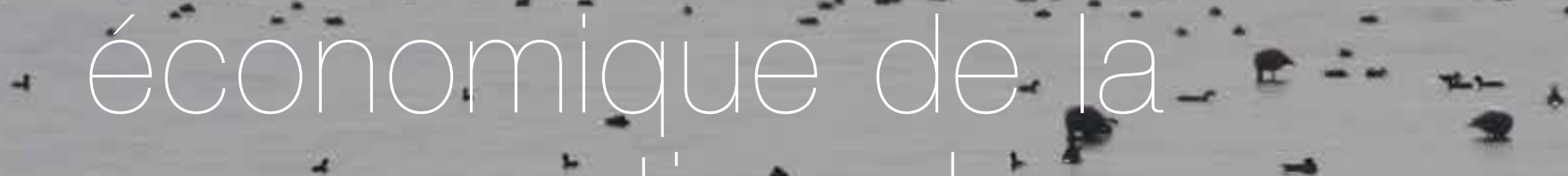
con'servation = dés habitats 'et de la + ",

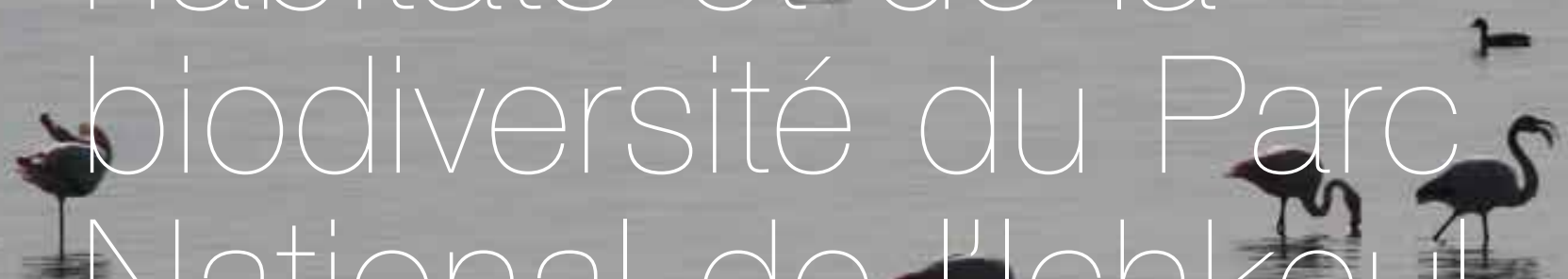

$\rightarrow \rightarrow \rightarrow$ ational a $\rightarrow$
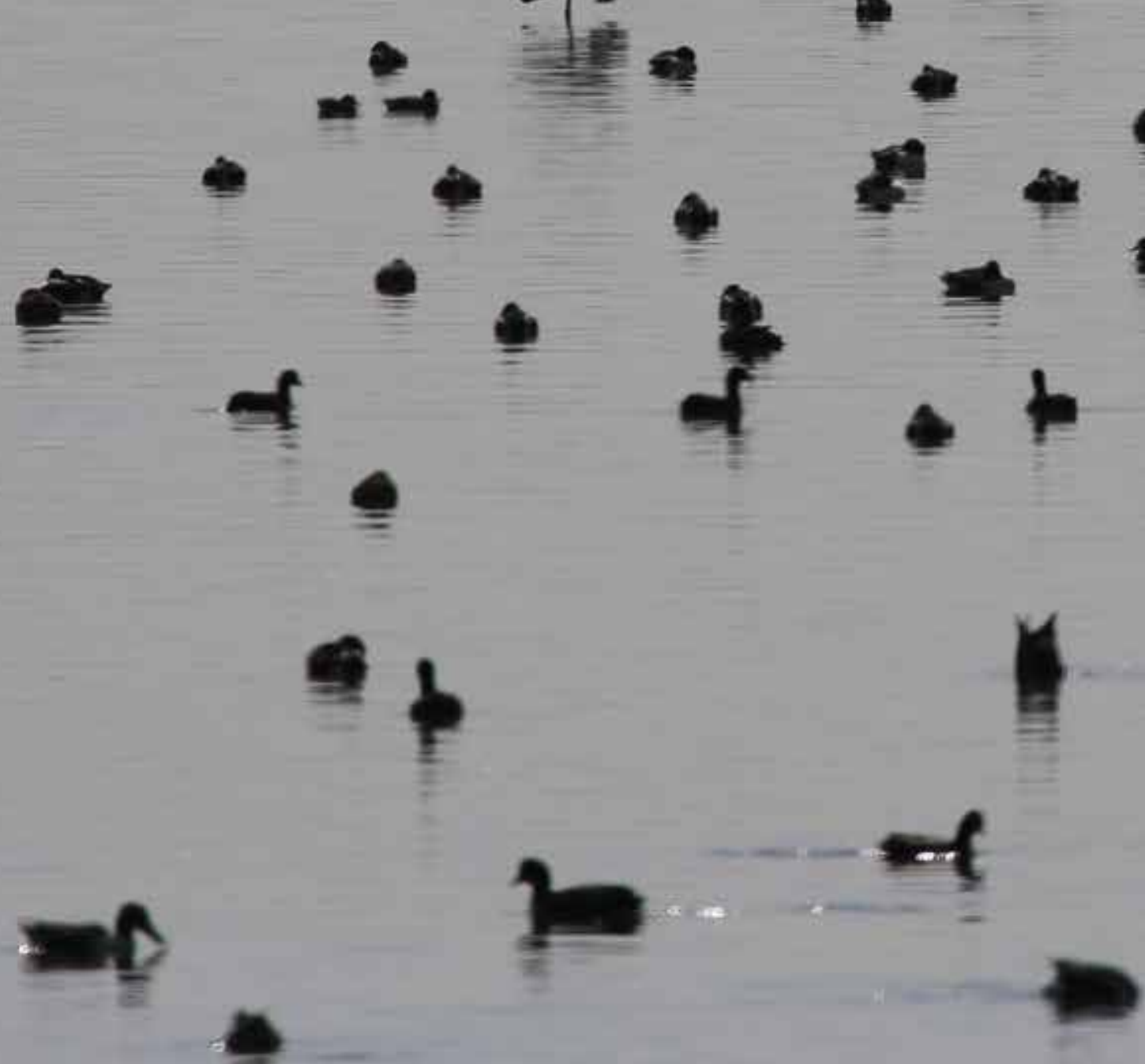
En plus des services écosystémiques à caractère utilitaire, l'évaluation économique de la conservation de la biodiversité, qui constitue à la fois une valeur d'usage et de non usage est présentée dans cette section. Cette évaluation économique est effectuée en utilisant la méthode d'évaluation contingente (MEC) à travers la disposition des populations concernées à payer pour la conservation de la biodiversité et des habitats. La biodiversité a un rôle en tant que fournisseur de services écosystémiques de manière durable.

Les différentes étapes de la MEC (Brahic et Terreaux, 2009) ont été appliquées comme suit :

- Etape 1 : Identification du changement à évaluer : la conservation de la biodiversité. II a été indiqué que l'administration du parc projette lélaboration d'un programm visant la conservation des habitats et de la biodiversite de l'écosystème de l'Ichkeul, avec ses trois sous-écosystèmes (lac, matas et montane). Le pogramme comporte tèmes (ac, mais et mola

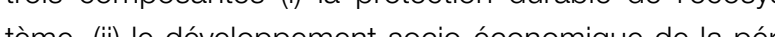
tème, (i) le divionpementa socio-economigue de la perpherie et visant la compensation des pertes de revenus (ii) un système de gestion efficace de l'eau du lac. Le premier paragraphe ci-dessous presente brievement les facteurs influençant la conservation de la biodiversité.

- Etape 2 : Identification de la population concernée : La population locale à l'intérieur et à l'extérieur du parc, les (1) bilité écologique. L'enquête a été effectuée auprès de trois

(3) échantillons distincts, à savoir : la population locale (100 chefs de ménage avec une différentiation entre la populan qui habite à l'intérieur du parc et celle de l'extérieur). s visiteurs du parc (93) ainsi que le grand public (43 indivius). Les deux premières catégories ont été interrogées sur le site étudié alors qu'un questionnaire en ligne a été ancé pour la dernière catégorie.

Etape 3: Choix du mode d'enquête : Enquête sur site (fac à face) et enquête hors site (en ligne sur internet) ; échantilns représentatifs pour les deux premiers groupes.

- Etape 4 : Etablissement d'un mode de paiement : Payement annuel par versement sous forme de donations dans un compte spécial géré, selon la réglementation en vigueur, par une ONG environnemental

Etape 5 : Rédaction et test du questionnaire d'évaluation. Après une introduction sur la nature et l'objectif du programme, une serie de questions a porte sur leurs avis sur ce programme et leurs dispositions à payer (cf. Annexe 4).

Etape 6 : Analyse des réponses. Les moyennes du maximum du consentement à payer des individus des différents échantillons sont calculées. La valeur correspond à la somme des dispositions à payer de toutes les population concernées par la conservation de la biodiversité, mêm s'ils n'en font aucun usage : Population locale, Visiteurs, public national et international (ayant un intérêt pour la protection de la biodiversité du parco.

6.1 Principaux facteurs influençant l'équilibre de l'écosystème

Lécosystème du lac Ichkeul est régi principalement par le ni- Les gestionnaires du parc Ichkeul et la plupart des études veau d'eau et la salinité, dont les valeurs extrêmes s'inversent notamment ANPE (1996), soulignent que l'implantation de au cours du cycle annuel : niveau d'eau bas en été avec des barrages (Sejnane, Joumine et Ghezala, Maleh récemmen niveaux de salinité plus elevés que celle de la mer, niveau d'eau mis en fonction) a provoqué l'augmentation de la salinité et la élevé en hiver avec une salinité proche de zéro (Tamisier 2007). baisse du niveau d'eau suite à la privation du lac des apports Ces fortes contraintes réduisent considérablement le d'eaux douces. A leur tour, ces facteurs affectent la productivité biologique primaire de l'écosystème (incluant les effets pur la flore aquatique et les habitats en aval et autres services écosystémiques). Ces impacts sont complexes, variés et Gearen gralement de cette ressource qui est leur nourriture de base: 2013). Pour cela, 'ANPE est chargée de la gestion de l'écuse 2007). Par ailleurs, à partir des variations observées amisier pour garder un maximum d'eau dans le lac en hiver et au de la période (1982-1993), Tamisier a montré une corréla- printemps et avoir une salinité faible de l'eau en été. tion négative entre l'effectif moyen annuel des canards et de foulques et le niveau d'eau moyen.

\section{Figure 10: Apports d'eau douce du lac (ANPE)}

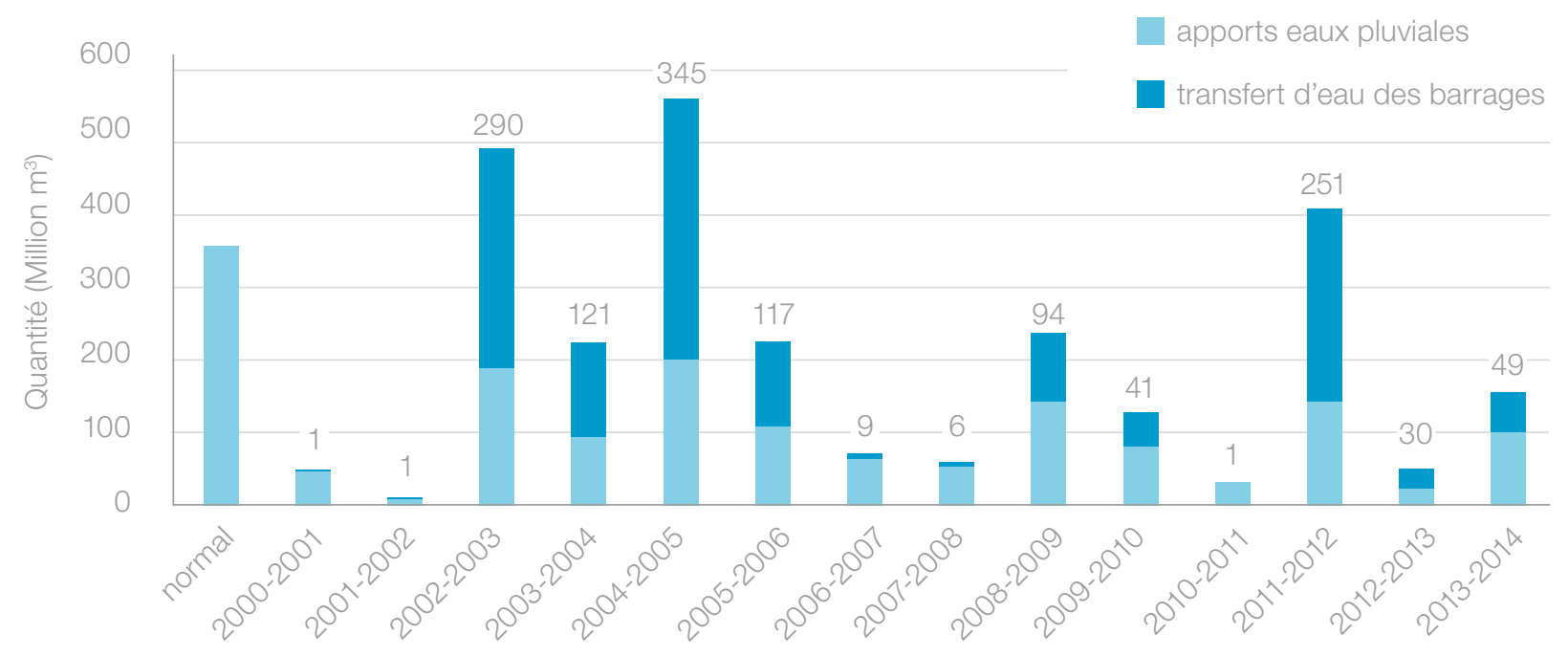

a figure 10 montre que les apports d'eau sont en dessous de la normale (situation avant la construction des barrages) à l'exception des années 2003, 2005 et 2012. Aussi, les apports d'eau des barrages sont souvent réduits lorsque les quantités d'eau du lac (apports sans lâchers) sont auss faibles (années de sècheresse : 2001-2002 ; 2007-2008 $2011 ; 2013)$, et vice versa. En effet, les lâchers d'eau sont effectuées en cas de crues et pour des buts de dévasement et non pas pour maintenir l'équilibre de l'écosystème Ichkeul. La demande environnementale connait des pics pendant les périodes sèches de l'année, où la salinité du lac peut nur les próćtation hydrique, et peut ainsi avor un effet nét nuire fréquentation du lac par les oiseaux migraurs (Fija 2000 . Pour remédier au manque d'eau durant les années de sècheresse, plusieurs études donnent des indications sur les quantités d'eau qu'il serait souhaitable de transférer au lac a partir des barrages.

Frija (2006), citant (ANPE 2003), a indiqué qu'un volume de $20 \mathrm{M} \mathrm{m}^{3}$ est envisagé d'être fourni, selon les années, par barrage Sidi Barrak, a travers le barrage Sejnane, une fois le remplissage de ce premier est terminé. Ghazouani (2013) recommande des apports supplementaires à partir du barrage Sidi Barrak de $90 \mathrm{M} \mathrm{m}^{3}$ afin d'éviter d'amplifier les problèmes de dégradation de l'écosystème dus à l'implantation des nouveaux barrages.

effet, les indices de setisfaction des différents compartments écologiques s'améliorent sensiblement avec cet apport en eau. Cependant, cette étude souligne le problème du coût élevé du pompage $\left(0,090 \mathrm{DT} / \mathrm{m}^{3}\right.$ en 2015, SECADENORD) pour le transfert d'eau de Sidi Barrak vers le barrage de Sejnane, souvent avancé par le Ministère de l'Agriculture.

e rapport ANPE (1996) a établi des scénarios écologiques à écluse gérées en fonction des apports en eau pour différents horizons de 1995 à 2015. Le rapport conclut que la satisfacion des contraintes écologiques dépend essentiellement volume total des apports annuels en eau douce au lac.

- Le taux de satisfaction du compartiment ${ }^{21}$ Potamogeton-oiseaux, qui correspond à la capacité d'accueil en canard et foulques, est inférieur à 15\% pour des apports annuels inférieurs à $250 \mathrm{M} \mathrm{m}^{3}$, il atteint un niveau de $100 \%$ pour un volume de 270 à $285 \mathrm{M} \mathrm{m}^{3}$.

- Le taux de satisfaction du compartiment Scirpes-Oies est deux mois, la salinité étant inférieure à $139 /$. $90 \%$ pour des apports de 240 à $270 \mathrm{M} \mathrm{m}^{3}$.

- Le compartiment poissons dépend des conditions de recrutement et des condifions du milieu interne du lac. Lensemble des contraintes du compartiment (recrutement des alevins, salinité, niveau du lac) est satisfait à partir d'un apport de $265 \mathrm{M} \mathrm{m}$

Thes est préservé dans tous les scénarios à écluse gérée. 
Les lâchers des eaux des barrages en cas de crues contri- que la salinité a atteint des pics (> $50 \mathrm{~g} /$ /) en 1995, 1997, buent largement à la baisse de la salinité des eaux du lac, 2000, 2001 et 2002 sur une durée de 12 ans (1995-2006). et ainsi à la restauration de la végétation succédant à une Malgré cette salinité élevée en 2001 et 2002, le niveau de période de sècheresse. A titre d'illustration, le rapport scien- production de poissons est resté élevé, 90 t environ (CRDA tifique de l'ANPE 2005/2006 indique que les niveaux de sa- Bizerte). On peut en conclure que des lâchers d'eau sont linité étaient inférieurs à $10 \mathrm{~g} / \mathrm{l}$ pour une période supérieure nécessaires durant les épisodes de sècheresse pour contrià deux mois pendant le printemps durant les années 2003- buer à la conservation de la biodiversité

2006, créant ainsi des conditions favorables pour le déve-

loppement des herbiers de potamots. La figure 11 montre

\section{Figure 11 : Evolution de la salinité moyenne des eaux du lac entre 1995 et 2006 (ANPE, 2006)}

(flèches en rouge signifie écluse fermée, fièches en vert signifie écluse ouverte)

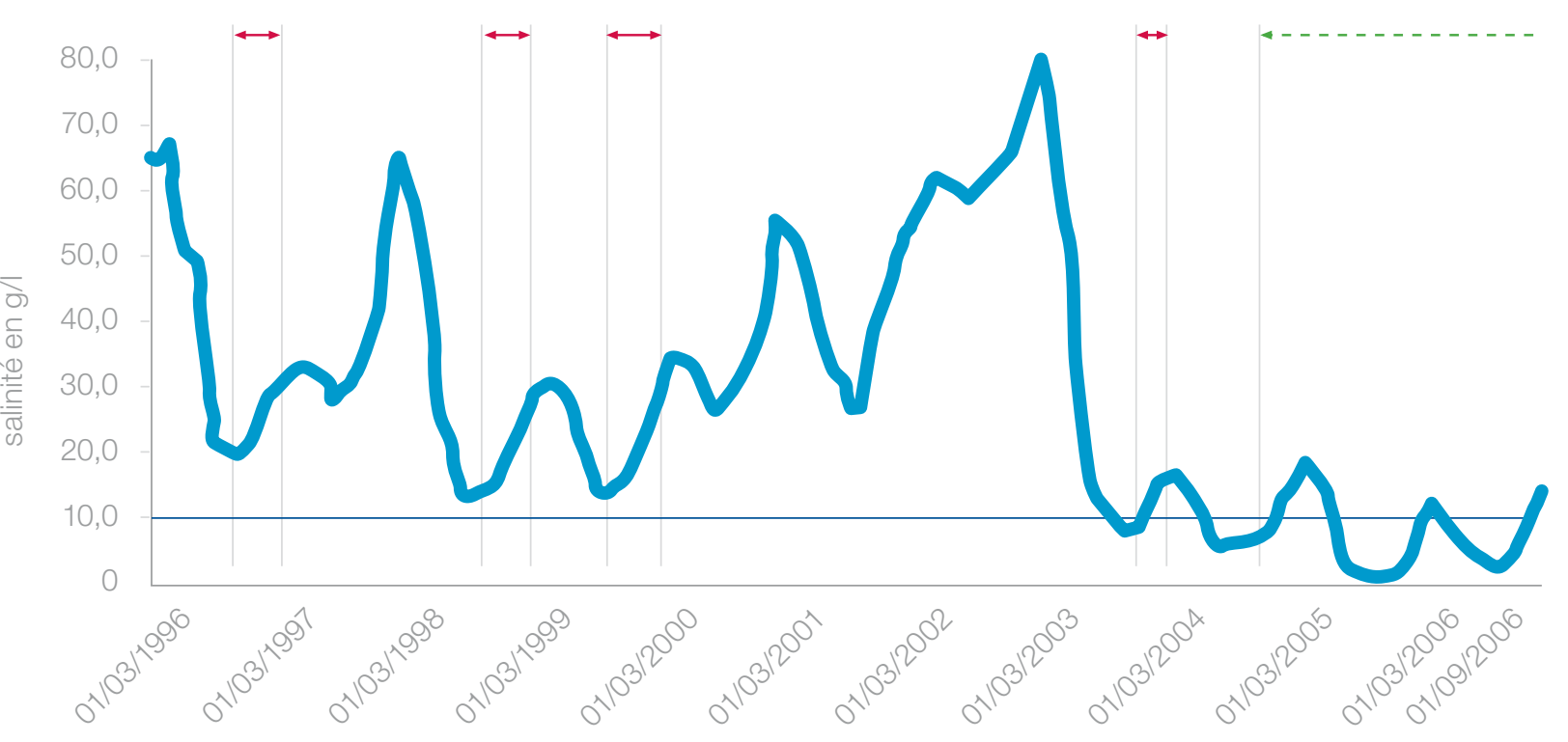

6.2 Résultats de l'enquête auprès de

la population locale et des visiteurs

Les résultats obtenus montrent que la totalité des personnes De même, la totalité des répondants appartenant à la populade l'échantillon de la population locale sont favorables à très tion locale ainsi que $72 \%$ des visiteurs sont disposés à payer favorables pour la réalisation du programme. Les visiteurs pour la mise en œuuvre du programme, tandis que $28 \%$ ont resont plutôt du même avis que la population locale avec 93\% fusé (cf. Tableau 40). En analysant les raisons de refus, il s'est favorables à très favorables pour la réalisation du projet, $4 \%$ avéré que $92 \%$ des réponses nulles correspondent à des faux sont indifférents alors que 3\% sont plutôt défavorables (cf. zéro représentant une réponse de contestation ('Ce n'est pas Tableau 39). der' 'Je ne paye pas pour un service «naturels - Don de Dieu-', 'Je crains de payer alors que d'autres en bénéficient').

selon l'échantillon. On note que $70 \%$ des visiteurs interrogés sur le site et $62 \%$ de la population locale voudraient que Lintérêt accordé à la conservation des différents sous-éco- la conservation de la biodiversité touche l'ensemble des systèmes (Montagne Lac, Marécage, Source d'eau) varie sous-écosystèmes en même temps (cf. Tableau 41).

\section{Tableau 39 : Avis de la population locale et des visiteurs sur un programme de conservation de la biodiversité}

Extérieur

Population Locale

Très favorable

$\begin{array}{ccc}\text { Nombre } & \text { Pourcentage } & \text { Non } \\ 50 & 100 \% & 46 \\ & & 4\end{array}$

Intérieur

Visiteur

Favorable

boureen

bre Pourcen

Avis sur Favorable $92 \%$

32
56 $34 \%$

\section{le projet Indifférent}

Défavorable

$4 \%$

Tableau 40 : Proportion de la population locale et des visiteurs disposés à payer pour un programme de conservation de la biodiversité

Disposition à payer

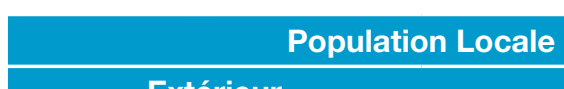

Extérieur

Population Locale

Vrai Zéro

Intérieur

Pource

50

$100 \%$

50

$100 \%$

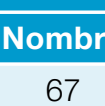

67

Faux Zéro 
Les personnes enquêtées appartenant à la population lo- Aussi, une question a été posée sur les types de service cale du parc, avec ses deux sous-catégories, se sont ex- écosystémiques pour lesquels les répondants sont dispoprimées avec plus de détails en accordant de l'importance sés à payer en vue de leur amélioration. La majorité des vià un sous-écosystème plus qu'à un autre. Les habitants du siteurs ayant accepté de participer à ce programme (43\%) parc (Intérieur) ont une préférence pour les sources d'eau souhaitent observer une amélioration des services liés aux ainsi que La Montagne et le Lac, alors que les habitants de activités de loisirs, suivis des services liés au 'Hammam' l'extérieur du parc (Extérieur), préêrent les marécages et le avec 26\% alors que 21\% souhaitent une amélioration de lac qu'ils portent un intérêt particulier. l'ensemble des services.

En ce qui concerne les espèces sauvages, $31 \%$ des visiteurs Le pâturage est le service le plus important selon la popuet 64\% de la population locale ont mentionné un intérêt pour lation locale de l'extérieur du parc (86\%) suivi du miel (76\%), la conservation de la faune et de la flore avec une préférence les services liés au hammam (70\%), le poisson et le bois. Les pour les espèces végétales de la part des habitants de l'ex- habitants de lintérieur du parc souhaitent une amélioration térieur du parc et une préférence pour les espèces animales liée à la production de bois $(96 \%)$, du pâturage $(94 \%)$, des (T) (cf. Tableau 42). produits aromatiques et médicinales (PAM 35\%) (Tableau 43).

Tableau 42 : Disposition à payer des répondants exprimée par type d'espèces

\begin{tabular}{llccc} 
& & \multicolumn{3}{c}{ Espèces Sauvages } \\
\cline { 3 - 5 } & & Animales & Végétales & Ensemble \\
\cline { 2 - 4 } Population à l'extérieur & $74 \%$ & $80 \%$ & $60 \%$ \\
\cline { 2 - 5 } Population à l'intérieur & Nourcentage & 37 & 40 & 30 \\
\cline { 2 - 5 } Visiteurs & Pourcentage & $94 \%$ & $71 \%$ & $68 \%$ \\
\cline { 2 - 5 } & Nombre & 47 & 35 & 34 \\
& Pourcentage & $63 \%$ & $41 \%$ & $31 \%$ \\
& Nombre & 59 & 38 & 29
\end{tabular}

Tableau 43 : Type de services écosystémiques à améliorer par le paiement (\% des réponses)

\begin{tabular}{lcccccc}
\multicolumn{1}{c}{ Population concernée } & Population à l'extérieur & Population à l'intérieur & \multicolumn{2}{c}{ Visiteurs } \\
\hline & Nombre & $\%$ & Nombre & $\%$ & Nombre & $\%$ \\
\hline Pâturage & 43 & $86 \%$ & & $94 \%$ & 1 & $1 \%$ \\
Bois & 10 & $20 \%$ & & $96 \%$ & 1 & $1 \%$ \\
PAM & 1 & $2 \%$ & 17 & $35 \%$ & 3 & $3 \%$ \\
Miel & 38 & $76 \%$ & 4 & $8 \%$ & 8 & $9 \%$ \\
Services relatifs au Hammam & 35 & $70 \%$ & & $90 \%$ & 24 & $26 \%$ \\
Poissons & 13 & $26 \%$ & & & 5 & $6 \%$ \\
Protection contre l'érosion & 3 & $6 \%$ & 8 & $16 \%$ & 3 & $3 \%$ \\
Loisirs & 3 & $6 \%$ & 8 & $16 \%$ & 40 & $43 \%$ \\
Tous les services & & & & & 20 & $21 \%$
\end{tabular}

CONSENTEMENT À PAYER (CAP) : Une fois les per- observés. On rappelle que la totalité des individus interrogés sonnes enquêtées ont décláé leurs dispositions à les perla réalisation du programme de conservation des habitas

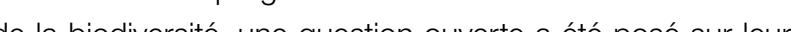

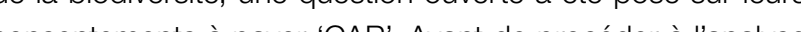
cos des vapurs anus, on a tout dabord procedé à rell mination nes valours aberantes (le CAP supérieur à $5 \%$ du revenu annuel moyen), ensuite les individus qui ne sont pas disposés a payer et dont les raisons ont été considérées comme des "vrais zéro" ont été pris en considération et finalement, pour éviter la grande variabilité des résultats obtenus, dû à la spontanéité des réponses, les CAPs ont été regroupés dans des intervalles comme le montre la figure 12

a plupart des visiteurs disposés à payer pour le programm conservation des habitas et de la biodiversité ont un CAP infeheur a $10 \mathrm{DT} / \mathrm{an}$ (25\%), aussi, $24 \%$ des visiteurs sont disposes a payer entre 20 et 50DT/an, tandis que $17 \%$ entre 10 et 20 DT/ an, $17 \%$ entre 50 et 100 DT/an et seulement $6 \%$ ont mentionné des valeurs supérieures à 100DT/an.

En ce qui concerne la population locale, $60 \%$ des habitants du parc ainsi que $48 \%$ des habitants à l'extérieur du parc on un CAP entre 20 et 50 DT par ménage, 13\% des habitants du parc et $24 \%$ des habitants a l'exterieur du parc ont un CAP entre 10 et 20DT, alors que13\% de líntérieur et 20\% de l'extéreur du parc ont un CAP entre 50 et 100DT. Des montants inferieurs à 10DT/an et supérieur à 100DT/an ont été faiblement

population locale sont disposés à payer.

Une étude de la corrélation entre le CAP d'un individu dispose a payer et un nombre de variables sociodémographiques a montre des differences marquantes entre le comportement des visiteurs et celui de la population locale.

Pour les visiteurs, le consentement à payer augmente avec le revenu annuel, qui peut se comprendre par le fait que plus e revenu est elevé plus lindividu peut se permettre de payer plus pour la realisation dun programme pour lequel il est favorable. La correlation entre l'etat matrimonial et le CAP a le même sens que le revenu, mais avec un impact plus faible peut s'expliquer par le sens de la responsabilité et l'amélioration du revenu par ménage. Contrairement à ces deux dernières variables le CAP est négativement corrélé avec l'âge a satisfaction de la visite effectuée le jour de l'enquête.

Sachant que $75 \%$ des visiteurs disposés à payer pour le programme sont âgés de plus de $35 a n s$, la correlation entre CAP et lâge peut s'expliquer par le fait que plus la personne est agee, plus elle a des charges et des dépenses et donc moins de moyens pour faire des contributions à un programme environnemental. En ce qui concerne la satisfaction de la visite, plus un individu est satisfait de la visite au parc, moins il est disposé à payer, probablement par crainte de

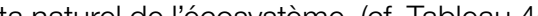

Tableau 44 : Valeurs du Consentement à payer 'CAP' (DT)

\begin{tabular}{cccc}
\hline CAP & Pop. Extérieur & Pop. Intérieur & Visiteurs \\
\hline 0 & 0 & 0 & $11 \%$ \\
\hline [0-10] & $4 \%$ & $11 \%$ & $25 \%$ \\
\hline $10-20]$ & $24 \%$ & $13 \%$ & $17 \%$ \\
\hline ]20-50] & $48 \%$ & $60 \%$ & $24 \%$ \\
\hline $50-100]$ & $20 \%$ & $13 \%$ & $17 \%$ \\
$>100$ & $4 \%$ & $2 \%$ & $6 \%$
\end{tabular}

Tableau 45 : Effet des variables sociodémographiques tement à payer des visiteurs

\begin{tabular}{|c|c|}
\hline \multicolumn{2}{|c|}{ Visiteurs } \\
\hline $\begin{array}{l}\text { Le Consentement } \\
\text { à payer augmente }\end{array}$ & $\begin{array}{l}\text { Le Consentement } \\
\text { à payer diminue }\end{array}$ \\
\hline $\begin{array}{l}\text { avec le Revenu Annuel } \\
\text { de l'individu (DT) (++) }\end{array}$ & avec I'Age (- -) \\
\hline $\begin{array}{l}\text { Si l'individu } \\
\text { est marié(+) }\end{array}$ & $\begin{array}{c}\text { avec la Satisfaction } \\
\text { de la visite (-) }\end{array}$ \\
\hline
\end{tabular}


En ce qui concerne la population locale à l'intérieur du parc, D'après les résultats observés, une diminution du revenu enle consentement à payer est positivement corrélé avec l'avis traine une augmentation du CAP, ceci peut être expliqué par sur le programme et le niveau d'éducation des personnes une dépendance au milieu naturel. L'enquête sur les usages du enquêtées, le sens de la corrélation suit une certaine logique parc a montré que $82 \%$ de l'échantillon étudié ont un revenu puisqu'un individu favorable à la réalisation du projet conser- issu de lélevage (activité directement liée au parc) et qui pour vation des habitas et de la de biodiversité a un CAP plus éle- $60 \%$ représente plus du tiers du revenu annuel par ménage. vé, de même qu'un individu plus instruit est plus conscien- Pour les habitants de l'extérieur du parc, la corrélation posicieux de l'environnement. La corrélation négative entre l'âge tive du revenu annuel par ménage et le CAP et celle négative et le CAP suit la même logique expliquée pour les visiteurs, tandis que le CAP diminue si la personne juge que l'etat du teurs. L'augmentation du CAP avec le niveau dinstrucion parc est bon (n'est pas degradé) (cf. Tableau 46). peut etre expliniee (come dans le cas des hablunts de

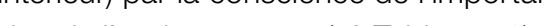
rel et de l'environnement (cf. Tableau 46.

Tableau 46 : Effet des variables sociodémographiques sur le consentement à payer de la population locale à l'intérieur du parc

\section{Population locale à l'intérieur du parc}

Le Consentement à payer augmente

Si l'avis sur le programme est favorable (++) avec le niveau d'éducation $(+)$ avec le Revenu Annuel du ménage (-)

Si l'état du parc est bon (-)

\section{Tableau 48 : Type de sous-écosystèmes à améliorer} par le paiement de la part du public (\% des réponses)
Lac

Marais

Montagne,

Montagne, Lac

Total enquete a eté lancée en ligne afin de viser les usagers ansi que les non-usagers du Parc National d'Ichkeul, cec se limitant à une population cible concernée par l'envinembres de l'Association des Amis des Oiseaux).

Le nombre de réponses reçues jusqu'au 25 janvier était de cis de I'ech $57 \%$ sont manies. Une repartion des efrec-

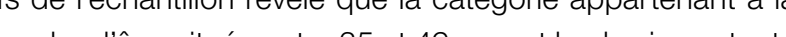
avec 35\%, alors que 30\% sont des jeunes dont l'âge est tue entre 25 et 34ans. Les personnes dont l'âge se situe 18-24ans et celle des plus de 65 ans sont lupart des enquêtés sont des cadres supérieurs $(43 \%)$ prs que $16 \%$ sont des étudiants, $26 \%$ des employés, $7 \%$ n, $21 \%$ ont un revenu annuel entre 2.000 et $10.000 \mathrm{DT} / \mathrm{an}, 35 \%$ . 50.000 et 50.000 , et $3 \%$ supériour à 50.000 DT

\begin{tabular}{ccc} 
& Nombre & Proportion \\
& 5 & $17 \%$ \\
\hline & 4 & $13 \%$ \\
\hline & 4 & $13 \%$ \\
rais & 16 & $53 \%$ \\
& & 1 \\
& 30 & $100 \%$ \\
\hline
\end{tabular}

Les personnes ayant répondu à l'enquête en ligne sont de differentes nationalités, ainsi on distingue 35 personnes (81\%) de nationalité Tunisienne, 3 de nationalité Française, 2 de nationalité Algérienne, 1 de nationalité Espagnole, 1 de nationalité Burkinabé, et 1 de nationalité Allemande.

CONSENTEMENT À PAYER : Une question a été posée dans le but de faire une distinction entre les personnes ayant visitees le parc national d'Ichkeul et celles qui ne l'ont jamais visité (valeur d'usage -valeur de non usage). Les résultats montrent que $81 \%$ des enquêtés ont déjà visité le site, et que parmi les 19\% restants (8 personnes), 7 ont lintention de le visiter.

Concernant le programme de conservation des habitas de la biodiversité, $81 \%$ des répondants (sur un total de 43 ) se sont montrés favorables à très favorables pour la réalisation du programme proposé, $9 \%$ indifferents alors que $10 \%$ sont putot defavorables a tres defavorables.

Indépendamment du fait qu'ils aient déjà visité le parc ou non, $70 \%$ des personnes (sur un total de 43 ) sont disposées a payer pour la réalisation du projet de l'amélioration de restants, il s'est avere que seuls $6 \%$ ont invoque des raisons exprimant des vrais zéro alors que pour le reste, ce sont des faux zéro qui représentent la contestation de l'interrogée ('Je n'ai pas assez d'informations pour me décider', 'Je ne paye

\section{Figure 13 : Valeurs du Consentement à payer 'CAP'
par la population nationale et internationale (DT)}

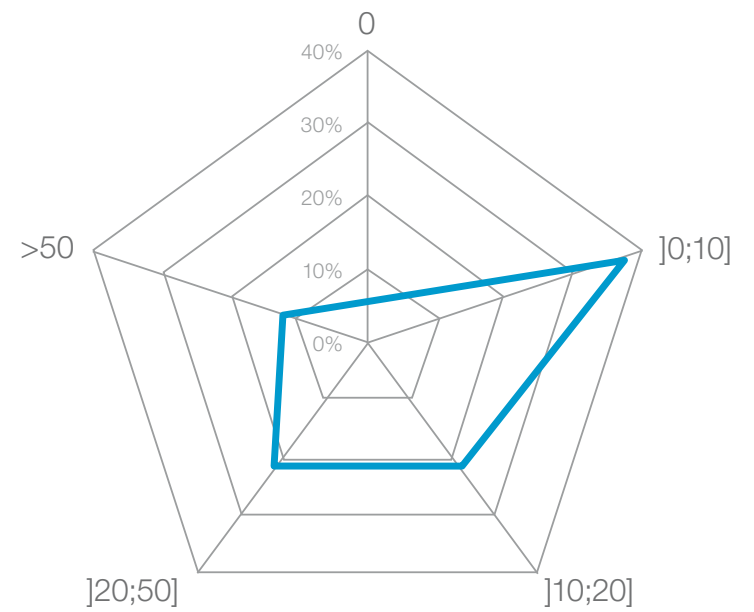




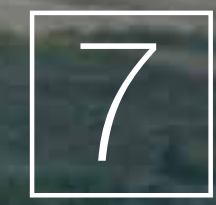

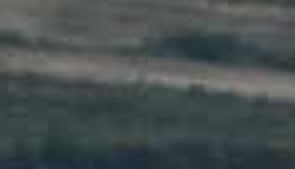

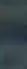

\section{Evaluation}

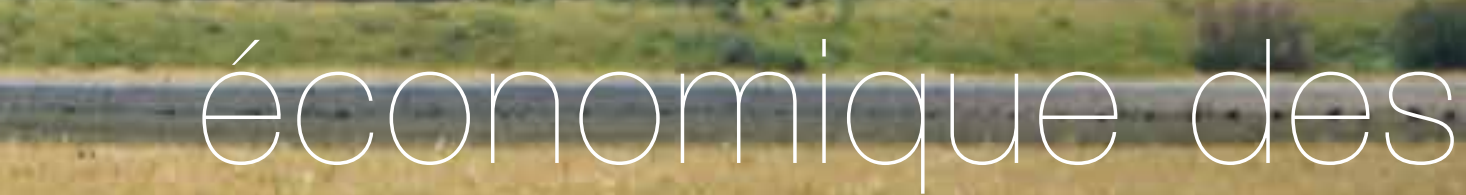
- programmes-de répeloiltetion idu

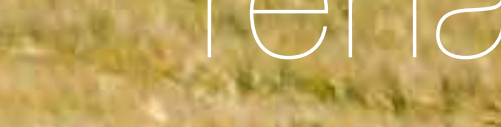
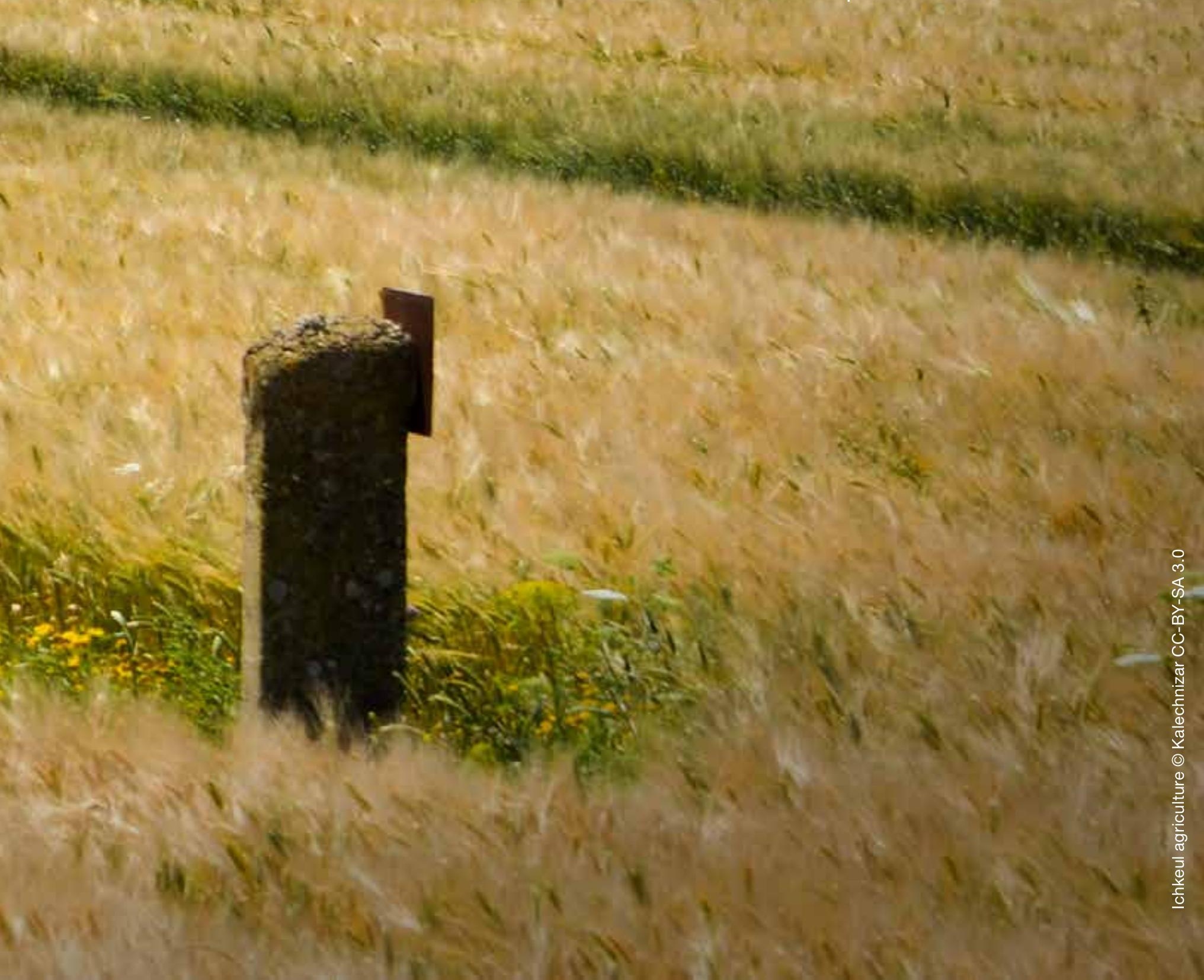

L'analyse économique des écosystèmes se donne souvent pour objectif de permettre aux décideurs d'effectuer des choix d'investissement ou de projets. Tout d'abord, l'évaluation des bénéfices sociaux permet de les comparer par rapport aux coûts nécessaires de la conservation de l'écosystème. Ensuite, lévaluation des pertes dues à des changements de la biodiversité permet d'arbitrer entre conservation et développement (Brahic, Terreaux, 2009). Ceci pourrait se faire par le moyen d'une analyse coûts-bénéfices qui pourrait déterminer les conséquences futures de choix d'aménagement. Afin qu'un programme soit choisi de manière rationnelle, il faudrait que les bénéfices soient simplement supérieurs aux coûts.

Les coûts annuels sont estimés ci-dessous avant de procé der à la comparaison.

Coût de gestion et de réhabilitation de l'écosystème. Le coût de gestion du parc est pris en charge par deux administrations : la DGF et l'ANPE.

- La DGF met en place un technicien, une équipe de gardiens (27 titulaires et 7 occasionnels) pour la préservation du parc et l'encadrement de l'activité écotouristique, soit un coût estimé à 199.000 DT environ en 2015. En plus, un budget annuel est alloué pour le fonctionnement du parc (49.000 DT en 2014) (CRDA Bizerte). Les frais d'unt vénher sont estimés à 10.000 DT par an. Ainsi les dépenses effecsont estimés à 10.000 DT par an. Ains

- Aussi, l'ANPE engage une équipe (3 ingénieurs) pour le suivi scientifique avec un coût estimé de 54.000 DT par an, et un coût de fonctionnement annuel de 100.000 DT an (ANPE). On ajoute à cela les frais d'un véhicule, estimés à 10.000 DT par an Ainsi, les dépenses sont estimées a 164.000 DT par an

La reconnaissance internationale et l'inscription du PNI sur les listes de conventions a facilité le financement de projets d'investissement dans le cadre de la coopération bilatérale et multilatérale, notamment le Fonds pour l'Environnement Mondial. En effet, dans le cadre d'un projet de Gestion des Aires protégées (GAP) (2005-2009), un investissement a été réalisé pour la réhabilitation du parc Ichkeul, en plus des aires protégées de Bouhedma et Jbil. Le coût du projet s'est éevevé à \$EU 9,88 million, équivalent à 14,8 Million $\mathrm{DT}^{22}$.

sonte proceder a cette comparaison, certaines données Le budget alloué pour la réhabilitation du PNI était de 2,6 pour assurer l'équilibre de l'écosystème? Est-ce que l'eau M DT (MARH /MEDD 2008). Ce projet visait notamment la serait utilisée au détriment de lírrigation ou non ? 
Il s'agit de comparer deux scénarios:

- Scénario de référence : Sans intervention : pas d'eau sup-

Les lâchers d'eau peuvent être justifiees economiquement Iorsque le Benéfice (Disposition a payer pour l'amelioration de la biodiversité) couvre le coût de preservation (valeur économique de l'eau). Comme la valeur de l'eau n'est pas

- Scénario avec intervention: Quantité d'eau additionnelle pour assurer la conservation de la biodiversite en année sèche. Ce scénario a deux variantes:

-Variante $1(V)$ : Cette quantité d'eau va être attribué aux dépens de l'agricultureiriguée(moins d'eau pour l'agriculth de l'eau est baś́e sur la perfomancéćconomic périmètres irrigués, soit $0,24 \mathrm{DT} / \mathrm{m}^{3}$. perise

作 s sont les suivantes:

- Variante 1(V1) : Q1 $<=16,4$ Million $\mathrm{m}^{3}$ (tous les 3 ans) Variante 2 (V2): Q2 $<=43,8$ Million $\mathrm{m}^{3}$ (tous les 3 ans) La quantité correspondante à la Variante 1 est légèrement plus faible que la demande environnementale de l'eau (20 Million $\mathrm{m}^{3}$ ) indiquée par ANPE (2003), alors que celle de la variante 2 la dépasse largement.

lorsque celui-ci atteint sa capacité maximale, l'eau se-

rait lâchee au lac au lieu d'être déversée dans la mer,

dans ce cas, on prend en compte seulement le coût de

pompage, soit 0, $09 \mathrm{DT} / \mathrm{m}^{3}$.

\section{8}

\section{Conclusions}

2
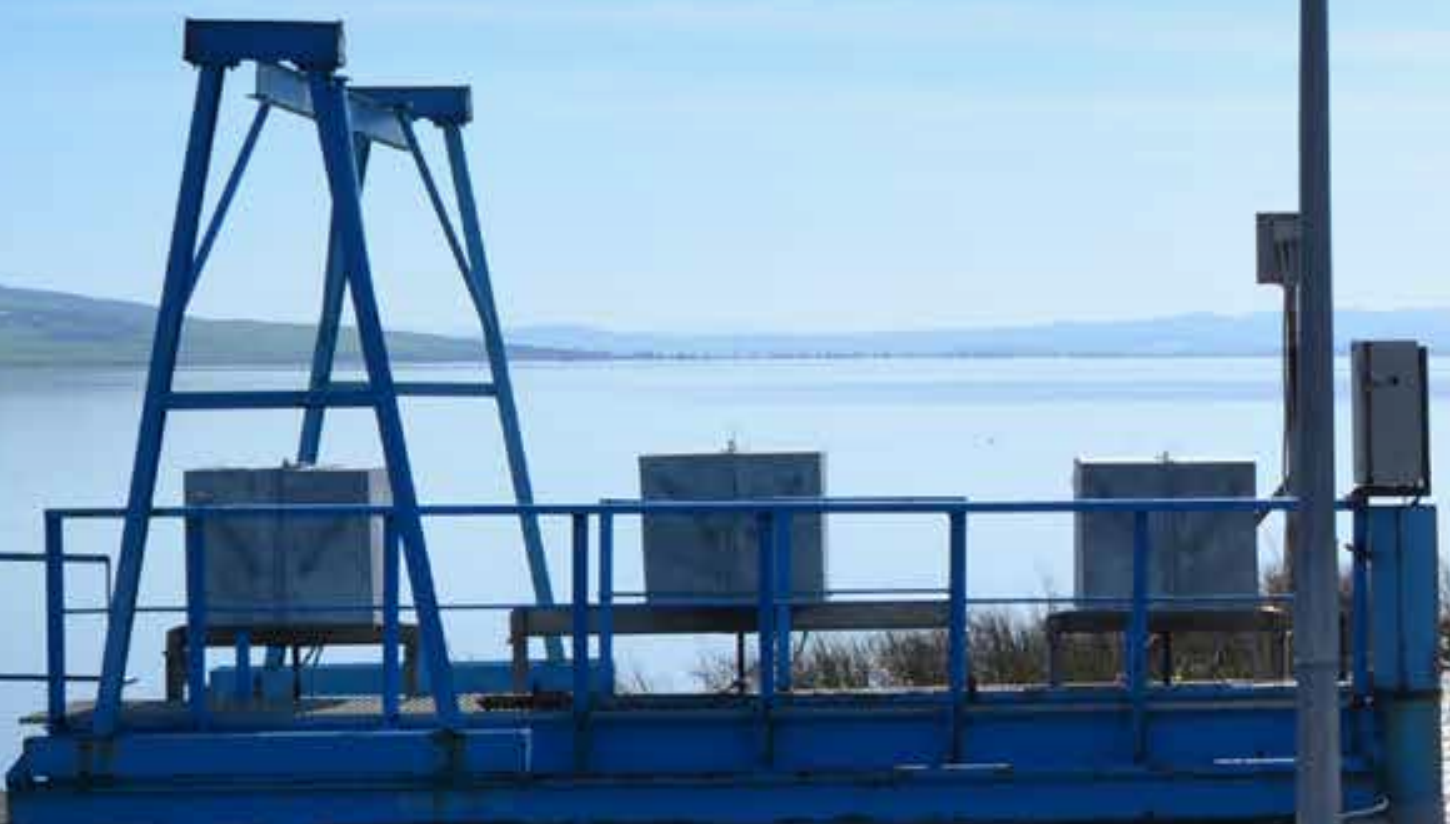

7
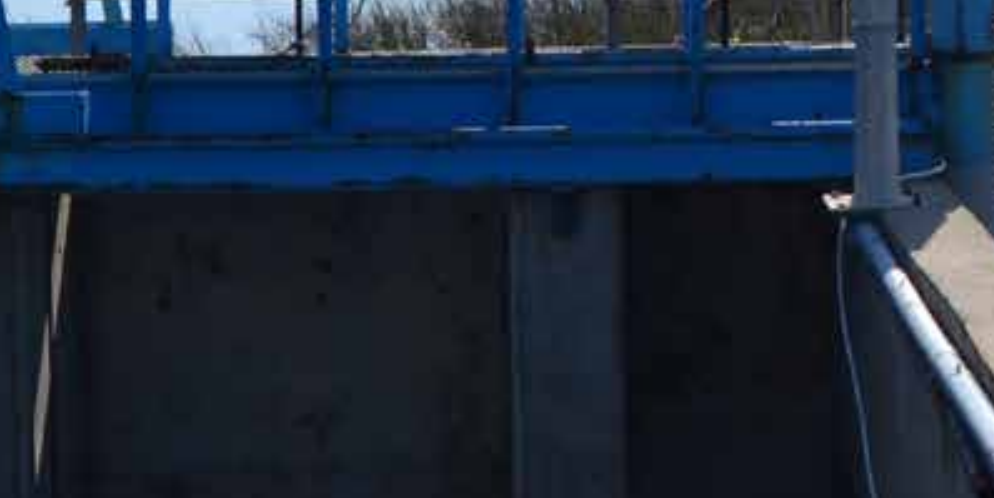
La compréhension de l'ensemble des valeurs des bénéfices fournis par le Parc National Ichkeul (PNI) est essentielle pour la prise de décision en matière de gestion. En effet, le PNI est source de bénéfices multiples, mais plusieurs d'entre eux ne sont pas reconnus alors que leurs valeurs économiques sont elevées. Ceux qui sont souvent appréciés sont la production de poissons et l'ecotourisme. La valeur des services écosystemiques obtenue est superieure a celle estimée récemmen pour les forêts tunisienne. En effet, le PNl est un ecosystem particulier en termes paysagers, écologiques, économiques grâce à la coexistence et aux interactions dynamiques entre les trois sous-écosystèmes. Aussi, il convient de noter les conflits entre certains usages (i.e. surpâturage, rétention des sédiments) et la conservation de la biodiversité ainsi que le problème de durabilité des flux des services écosystémiques suite à l'accroissement du nombre de barrages.

Les composantes et la multifonctionnalité du PNI sont considérées comme un moteur de développement local voire régional. Cette étude a montré aussi la diversité des utilisateurs directs et indirects du parc. La population locale considère parc comme un habitat, une source de revenus et de loisirs. Aussi, les agriculteurs, notamment I'OTD, et les habitants de b ville de Mateur sont concernés par la protection contre les inondations, alors que l'entrepre príe est intesse par i production de poissons. De mene, les habits aes par la production de poissons. De meu, les habitants des communes voisines (Tinja, Menzel Bourguiba, Mateur) notamment, sinteressent à leccotourisme et à la frequentation du hammam.

Lenquête effectuée auprès de la population locale sur la disposition à payer pour l'amélioration de la biodiversité de lécosystème Ichkeul montre une position très positive, toufofis, cette attitude est à base utilitaire, relative à l'amélioraton des services écosystémiques (pâturage, services liés au hammam, etc.). L'enquête auprès des visiteurs présente un aspect moins utilitaire, en effet, certains visiteurs ont exprim leur disposition à payer pour l'amélioration de la biodiversité et non pas pour tirer profit de ses services. L'enquête auprès du public a montré aussi une forte disposition à payer que l'on peut considérer comme une valeur de non usage en grande partie. La démonstration des valeurs d'existence au niveau international permet de justifier les prêts et donations internationales pour la protection du parc. L'amélioration de a conscience environnementale dans le futur contribuerait à une meilleure perception de l'amélioration de la biodiversité par une part plus large de la population.
Les bénéfices économiques, que ce soit la valeur des services écosystémiques ou la valeur de l'amélioration de la biodiversité, excèdent le coût de préservation du parc. Ainsi, les dépenses consenties par l'Etat pour la conservation du parc ont justifiées économiquement. De plus, la valeur attribuée l'amélioration de la biodiversité pourrait justifier les lâchers d'eau dans le lac (15 à 41 Million $\mathrm{m}^{3}$ tous les 3 ans).

Ceci nous amène à formuler les recommandations suivantes dans le but d'un accroissement des services fournis par le PNI pour la société :

Renforcer la contribution du lac dans la protection des terres agricoles en amont et de la ville de Mateur contre les inondations. Ceci signifie un entretien du réseau d'assainissement des terres agricoles périphériques, une réduction de la sédimentation du lac pour emmagasiner plus d'ea dans les périodes de crues et d'inagtions. II faudreit signar qu'actuele cent, une supericie de 1.000 ha envion est encore sounise à des rinfices dinondation malgé le dévere soumise à des risques dinondation malgre le deversement des eaux dans lac. D'autant plus, ce role benéfique pour la population de la région est accompagné par une amélioration l'équilibre de l'écosystème lacustre.

Développer la contribution du PNI aux bénéfices de société. Certains services comme l'écotourisme, léducation environnementale, le nettoyage, la relaxation et le soin au hammam, pourraient avoir des valeurs beaucoup plus élevées avec une gestion plus adéquate répondan aux attentes des bénéficiaires. La participation de toutes les parties prenantes : Résidents du parc, des riverains, des agriculteurs de la région, notamment l'OTD, les responsables de la commune de Mateur et des services de gestion, est necessaire afin que le lac contribue de manière optimale au contrôle des inondations et à la rétention des sédiments, tout en assurant une bonne production de poissons. De même, la récréation et les services éducatiffs devraient être développés (facilité des moyens de transport par exemple) avec les communes voisines, dans les limites du respect de l'environnement. L'amélioprenates de mieux proffer aes services du parties porter atteinte à l'équilibre de l'écosystème.

Considérer les bénéfices multiples du PNI dans la gestion de l'écluse, et ne pas se focaliser simplement sur les conditions favorables pour développer un service particulier. Plusieurs services rendus par le parc ne sont pas recon Pus alors que leurs valeurs économiques sont
4 Mieux tirer profit des services du parc et rechercher des solutions de compromis entre les differents acteurs sur les différents usages, et ce sans porter atteinte à l'équilibre de l'écosystème, à travers la logique gagnant-gagnant, le développement local et la cogestion des ressources naturelles. Par exemple, la valeur du service des hammams, qui constitue la principale attente de la polution du lac. De meme, le pâtura gui peut etre mieux géf en assuran un bon compromis ente les hama

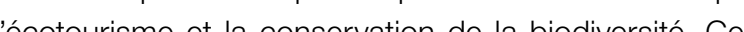
recotounisme et la conservation de la biodiversite. Ces paromis, notamment à travers des mécanismes de paiement des services, permettront non eulement dassurer une coordination entre les differents bénéficiaires sur la gestion du parc, mais aussi de garantir un meilleur partage des coûts et des bénéfices.
Mettre en place des mecanismes et dispositifs pour assurer les paiements de certains services environnementaux par les bénéficiaires, ou au moins prendre en charge les coûts des activités (visiteurs, agriculteurs, etc.). Cette étude a montré la disposition des visiteurs à payer un droit d'entrée au parc de 25 DT en moyenne pour les adultes et de 1 DT pour les enfants par visite.

Orienter l'approche de conservation du PNI vers des arguments economiques bases sur le maintien, voire 'optimisation de la production des services écosystém ques fournis, pour justifier les mesures de conservation du parc, complémentaire avec l'approche éthique de préservation de la biodiversité. La conception des programmes et des projets de protection du PNN nécessite une nouvelle vision en rapport avec le développemest versants. 


\section{Références}

Agence de l'eau Adour-Garonne, 2009. Evaluation économique des zones humides. Volume 1 : Synthèse de la bibliographie. Eco What/Acteon, France.

ANPE, 1996.Etude pour la sauvegarde du Parc National de 'Ichkeul. Plan de gestion optimale du parc de l'Ichkeul.

ANPE, 2003. Plan de sauvegarde de l'lchkeul.

Aouididi 1996. Evaluation économique du parc nationa d'Ichkeul, Université d'Ottawa, Canada.

Banque mondiale, 2005. Evaluer les coûts de la dégradation de l'environnement, élaboré par K. Bolt, G. Ruta et M. Sarraf Document $n^{\circ} 106$.

Banque mondiale, 2007. Tunisie : Evaluation du coût de dégradation de l'eau, élaboré par M. Sarraf, L. Croitoru, F. Ghariani, M. Mattoussi et H. Daly-Hassen. Washington.

Ben Belgacem W., 2013. Mesure de l'indicateur de suivi : Services récréatifs et éducatifs des zones humides méditerranéennes: Cas du Parc National de l'Ichkeul en Tunisie, Mastère de recherche, ESA Chott Meriem.

Men Mbarek N., 2001. Etude l'ecosystème du lac Ichkeul de son bassin versant : Caractérisation physique et géoch mique des eaux et des sediments. Faculte des Sciences de Tunis.

Benessaiah N., 1998. Aspects socio-économiques des zoes humides méditerranéennes

Bishop J.T. (Editor), 1999. Valuing forests : A Review of Methods and Applications in Developing Countries, IIED, London. Bontems P., Rotillon G., 2007. L'économie de l'environnement, Troisième édition, Editions La découverte, Paris.

Brahic E., Terreaux J.P., 2009. Evaluation économique de la biodiversité : Méthodes et exemples pour les forêts tempérées. Editions Quae, France.

Brander L. and Schuyt K. (2010). TEEB case study : The economic value of the world's wetlands available at : TEEBweb.org.

Centre d'Analyse Stratégique (CAS), 2009. Approche économique de la biodiversité et des services liés aux écosystèmemique de la biodiversité et des services liés aux
Chennoufi S., 1995a. Quels espaces possibles pour les auIntones de l'Ichkeul ? I : Le parc et les intervenants. Cahier du CERES, série sociologique n`24, p. 167-202

Chennoufi S., 1995b. Quels espaces possibles pour les autochtones de l'chkeul ? II : Les alternatives. Cahier du CERES, série sociologique n²4, p. 113-151.

CRDA Bizerte 2009. Suivi de la mise en œuvre du plan d'aménagement et de gestion du PNI, décembre 2009 .

CRDA Bizerte, 2006. Plan de développement communautaDaly-Hassen H. 2013. Guide méthodologique : Evaluation économique des biens et services des écosystemes. Cas de la subéraie et des nappes alfatières en Tunisie. GIZ, Tunis. Darwall W., Carrizo S., Numa C., Barrios V., Freyhof J. et Smith K. (2015). Les Zones clés pour la biodiversité d'eau douce dans le hotspot du bassin méditerranéen. Un éclarage pour la conservation des espèces et la planification du developpement dans les écosystèmes d'eau douce. Cambridge, Royaume-Uni et Malaga, Espagne : UICN. 86pp. httos : Mportals.iucn.org/library/sites/library/files/documents/s/ SSC-OP-052-Fr.pdf.

De Groot R., Brander L., van der Ploeg S., Costanza R. Bernard F., Braat L., Christi, M., Crossman N., Ghermand A., Hein L., Hussain,S., Kumar P., McVittie A., Portela R., Rodriguez L.C., ten Brink P., van Beukering P., (2012). Global estimates of the value of ecosystems and their services monetary units. Ecosystem Services 1, 50-61.

DG Pêche, annuel. Statistiques de la pêche en Tunisie, annuel, 2000 à 2014.

DGF, 2015. Atlas des aires protégées en Tunisie, Ministère de

Direction Générale des Forêts (DGF), 2006. Plan d'Amén gement et de Gestion du Parc National de l'chkeul et de sa périphérie, MARH.

Ferchiou A., 2011. Contribution à l'analyse de la valeur écomique d'un espace naturel protégé : Cas du parc nationa d'Ichkeul, Projet de fin d'études, INAT.
Frija A. 2006. Gouvernance et allocation de l'eau entre usages sur l'écosystème d'Ichkeul, Projet de fin d'études, INAT/CERTE

Juffe-Bignoli D. et Darwall W.R.T (éds.), 2012. Évaluation de a valeur socioéconomique des espèces d'eau douce en $A$ rique du Nord. Gland, Suisse et Malaga, Espagne : UICN MED. IV + 92 pages. https : //portals.iucn.org/library/efiles/ documents/2012-042-Fr.pdf.

King.M.D., Wainger LA, 2001. Assessing the economic value of biodiversity using indicators of site conditions and andscape context In Valuation of biodiversity berefits : Seected studies, OECD, France, p.121-150.

MARH/MEDD. 2008. Rapport d'avancement du projet Gestion des Aires protégées, Tunis

Merlo M., Croitoru L. (Eds.), 2005. Valuing Mediterranean forests : Towards total economic value. CABI Publishing, Wallingford, Oxon, $406 \mathrm{p}$.

Millenium Ecosystem Assessment, 2005. Ecosystems and human well-being. Current state and trends assessment, Washington D.C., Island Press.

Ministère de l'Equipement et de l'Environnement 2013. Tunisie : Seconde Communication Nationale à la Convention Cadre des Nations Unies sur les Changements Climatiques. http : //unfccc int/resource/docs/natc/tunnc2 pod.

Monke, E.A. and S.R. Pearson. 1989. The Policy Analysis Matrix for Agricultural Development. Cornell University Press, Ithaca, New York.

Observatoire des zones humides méditerranéennes, 2012 Les zones humides méditerranéennes : Enjeux et perspectives. Rapport technique, Tour de Valat, France, 128p.

RAMSAR, 2012. Les zones humides et le tourisme: Tunisie - Lac Ichkeul http : //www.ramsar.org/sites/default/files/documents/ pdf/case studies tourism/Tunisia/Tunisia/chkeul FR.pdf

République Tunisienne, 2011. Code forestier et ses textes d'application, Imprimerie officielle, Tunis.
Russi D., ten Brink P., Farmer A., Badura T., Coates D., FörsRer J. Kumar R. and Davidson N. (2013). The Econ. ForsEcosystems ondon and Brussels; Ramsar Secretariat, Gland.

Salles, J-M., Évaluer la biodiversité et les services écosystèmiques : pour quoi faire ? Document de recherche. LAMETA, CNRS, Montpellier.

Schuyt, K., And Brander, L. 2004. The Economic Values of the World's Wetlands, Gland/Amsterdam, WWF.

Staub C., Ott W. et al. 2011 : Indicateurs pour les biens et services écosystémiques : Systématique, méthodologie et recommandations relatives aux informations sur l'environnement liées au bien-être. Office fédéral de l'environnement, Berne. L'environnement pratique $n^{\circ} 1102: 14$ p.

Tamisier A., 1997. Les oiseaux d'eau, Indicateurs de changements d'habitats. Le cas de l'Ichkeul. Actes du séminaire "Gestion et conservation des zones humides tunisiennes ", projet MEDWET, Sousse, octobre 1997, p. 91-98.

TEEB (2010), The Economics of Ecosystems and Biodiversity : Ecological and Economic Foundations. Edited by Pushpam Kumar. Earthscan, London and Washington.

Thomas D. H.L., Ayache F. and Hollis G.E., 1991.Use and Non-use Values in the Conservation of Ichkeul National Park, Tunisia, Environmental Conservation. Volume 18, Issue 02. Turner, K., den Bergh, J., Soderquist, T., Baerendregt, A derStraaten, J Maltby, E and Van lerland, E. (2000) Ecolo(ical economic analysis of wetlands, Ecological economics $35: 7-23$. UICN/CEPF, 2015. Analyse de linformation sur le Parc Nationa de I'Ichkeul a Bizerte et la Sebkha Sidi Mansour à Gafsa et renforcement de leur partenariat avec les institutions de recherche et les organisations de la société civile en Tunisie, DGF, Tunis.

UICN-Med, 2015. Plan de gestion des visiteurs du parc national d"Ichkeul. Projet « MEET - Expérience méditerranéenne d'écotourisme, ENPI CBCMED.

Zaiane S., 2004. Tourisme et loisirs dans les parcs nationaux tunisiens. L'exemple du Parc National d'Ichkeul. Centre de Publication universitaire. 
Les usages du parc - année 2015

\begin{tabular}{|l|l|l|}
\hline & Produits et services & Pour la vente \\
\hline & Pour l'usage familial & \\
\hline Forêt & & \\
\hline Eaux thermales & & \\
\hline Jardin familial & & \\
\hline Marais & & \\
\hline Lac & & \\
\hline Autres & & \\
\hline
\end{tabular}

\section{Produits agricoles en 2015}

\begin{tabular}{|l|l|l|l|l|}
\hline Type de culture & Surface & Source d'eau & Recettes DT/an & Dépenses DT/an \\
\hline & & & & \\
\hline & & & & \\
\hline
\end{tabular}

\section{Pêche à titre individuel en 2015}

\begin{tabular}{|l|l|l|l|l|l|l|}
\hline Type de pêche & $\begin{array}{l}\text { Type de } \\
\text { poissons }\end{array}$ & Période & Fréquence & Quantité & Prix & $\begin{array}{l}\text { Coût de } \\
\text { production }\end{array}$ \\
\hline & & & & & & \\
\cline { 2 - 7 } & & & & & & \\
\cline { 2 - 7 } & & & & & & \\
\hline
\end{tabular}

Autres usages du parc (médicinal, etc.) en 2015

\begin{tabular}{|l|l|l|l|l|}
\hline $\begin{array}{l}\text { Espèces animales } \\
\text { / végétales }\end{array}$ & $\begin{array}{l}\text { Type de zone } \\
\text { (Jbel, lac, marais) }\end{array}$ & Usages & Q collectée/ an & Revenu (DT/an) \\
\hline & & & & \\
\hline & & & & \\
\hline
\end{tabular}

\section{Activités salariées (chef du ménage et membres de la famille) en 2015}

\begin{tabular}{|l|l|l|}
\hline & Activité & Revenu annuel (DT/an) \\
\hline A l'intérieur du parc & & \\
\hline En relation avec le parc & & \\
\hline Horsdu parc & & \\
\hline
\end{tabular}

\section{Produits forestiers}

\begin{tabular}{|l|l|l|l|l|l|l|l|l|}
\hline & S (ha) & $\begin{array}{l}\text { Période } \\
\text { d'exploit. }\end{array}$ & $\begin{array}{l}\text { Fréq. (Nbre } \\
\text { /semaine) }\end{array}$ & $\begin{array}{l}\text { Production } \\
\text { (Kg/j) }\end{array}$ & $\begin{array}{l}\text { Part } \\
\text { consommé }\end{array}$ & $\begin{array}{l}\text { Lieu de } \\
\text { vente }\end{array}$ & Prix & $\begin{array}{l}\text { Membres } \\
\text { impliqués }\end{array}$ \\
\hline Olives & & & & & & & & \\
\hline Câpres & & & & & & & & \\
\hline Caroubes & & & & & & & & \\
\hline Bois & & & & & & & & \\
\hline Lentisque & & & & & & & & \\
\hline Autres & & & & & & & & \\
\hline
\end{tabular}

Pâturage

\begin{tabular}{|l|l|l|l|l|l|l|l|l|l|l|l|l|}
\hline mois & 1 & 2 & 3 & 4 & 5 & 6 & 7 & 8 & 9 & 10 & 11 & 12 \\
\hline Montagne & & & & & & & & & & & & \\
\hline Marais & & & & & & & & & & & & \\
\hline $\begin{array}{l}\text { Nbre } \\
\text { d'h par j }\end{array}$ & & & & & & & & & & & & \\
\hline Membres impliqués (H, F, J) & & & & & & & & & & & & \\
\hline Lentisque & & & & & & & & & & & & \\
\hline Autres & & & & & & & & & & & & \\
\hline
\end{tabular}

Production animale

\begin{tabular}{|l|l|l|l|l|}
\hline mois & Bovins & Ovins & Caprins & Abeilles \\
\hline Nombre (têtes /ruches) & & & & \\
\hline Bénéfice net par unité & & & & \\
\hline Bénéfice net par an & & & & \\
\hline $\begin{array}{l}\text { Membres impliqués } \\
\text { (H, F, J) }\end{array}$ & & & & \\
\hline Lentisque & & & & \\
\hline Autres & & & \\
\hline
\end{tabular}

\section{Eaux thermales}

\begin{tabular}{|l|l|l|l|l|l|l|l|l|l|l|l|l|}
\hline mois & 1 & 2 & 3 & 4 & 5 & 6 & 7 & 8 & 9 & 10 & 11 & 12 \\
\hline Fréquence (Nbre/semaine) & & & & & & & & & & & & \\
\hline Nbre de membre de famille & & & & & & & & & & & & \\
\hline
\end{tabular}




\begin{tabular}{|c|c|c|}
\hline $\begin{array}{c}\text { Nom } \\
\text { Scientifique }\end{array}$ & Nom Français/ Arabe & Utilisation \\
\hline Cichorium intybus & Chicorée sauvage (chkouria) & diabète \\
\hline $\begin{array}{l}\text { Scorzonera } \\
\text { undalata vahl }\end{array}$ & Scorzonere (guiz) & dépuratif, brülures \\
\hline Sonchus oleraceus & Laiteron Commun (difef) & verrues, leucomes oculaire, favorise la lactation, \\
\hline & difef) & dépuratif et antidiabétique \\
\hline Erica multiflora & bruyère multiflore (kchleni) & cancer de la prostate \\
\hline Arbutus unedo & Arbousier (boujbiba) & $\begin{array}{l}\text { antidiabétique, hypotenseur, certains cancers, } \\
\text { antidiarrhéique }\end{array}$ \\
\hline Mercurialis annua & $\begin{array}{l}\text { Mercuriale annuelle (h'baq el } \\
\text { dhol) }\end{array}$ & stérilité et frigidité \\
\hline Ricinus communis & Ricin (kharouáa) & $\begin{array}{l}\text { bronchites, usages externes, fièvre, thumatisme, } \\
\text { acné }\end{array}$ \\
\hline Fumaria capreolata & \begin{tabular}{|l} 
Fumeterre (sibana) \\
\end{tabular} & diverses dermatoses \\
\hline Globularia alypum & Globulaire (zriga) & $\begin{array}{l}\text { cicautrisantes, acné, eczéma, ulcère gastrique, } \\
\text { antipaludique, antirhumatismale, cancer de la } \\
\text { peau }\end{array}$ \\
\hline Ajuga iva & Ivette musquée (chandgoura) & antidiabètique, hypertension, toux \\
\hline Marrubium vulgare & Marrube blanc (omerroubia) & $\begin{array}{l}\text { antidiabètique, hémoroides, antirhumatismale, } \\
\text { maladies gastriques, abcés }\end{array}$ \\
\hline Mencha pulegium & Menthe pouliot (llayou) & toux, céphalée, gastralagie, aérophagie, asthme \\
\hline $\begin{array}{l}\text { Mentha } \\
\text { rotundifolia }\end{array}$ & $\begin{array}{l}\text { Menthe à feuilles rondes } \\
\text { (marsita) }\end{array}$ & usages extermes contre abcés, furoncles \\
\hline $\begin{array}{l}\text { Rosmarinus } \\
\text { officinalis }\end{array}$ & Romarin (klii) & $\begin{array}{l}\text { troubles hépatiques, thumatisme, laxatif, asthme, } \\
\text { cicartisation }\end{array}$ \\
\hline Myrtus communis & Myrthe (rihan) & ulcètr, douleurs gastriques, thumatisme \\
\hline $\begin{array}{l}\text { Centaurium } \\
\text { pulchellum }\end{array}$ & $\begin{array}{l}\text { Petite centauré (quosset } \\
\text { sbiya) }\end{array}$ & dépurative \\
\hline
\end{tabular}

\begin{tabular}{|c|c|c|}
\hline $\begin{array}{c}\text { Nom } \\
\text { Scientifique }\end{array}$ & Nom Français/ Arabe & Utilisation \\
\hline Agave americana & Agave (Sabbara) & $\begin{array}{l}\text { les feuilles ont des vertus antirhumatismales et } \\
\text { anti-infectieuses }\end{array}$ \\
\hline Arum Ivalium & Arum d'Talie (Sabbat ghoula) & antiseptique et anti-infectieuse. \\
\hline Arundo donax & Canne de Provence (ksab) & $\begin{array}{l}\text { diurétique dans les parties aériennes de cette } \\
\text { plante. }\end{array}$ \\
\hline Cyrodon dactylon & Gros chiendent (nejm) & diurétique/antirhumarisme/ antidiabéique \\
\hline Phoenix dactyliffera & Palmier dartier (nakhla) & énurésie/gerçures \\
\hline $\begin{array}{l}\text { Mesembryanthenu } \\
\text { m edule }\end{array}$ & Figue marine (charbabbou) & propriétés cicatrisantes/ anti-verrues \\
\hline $\begin{array}{l}\text { Pistacia lentiscus } \\
\end{array}$ & Lentisque (dharou) & $\begin{array}{l}\text { douleurs gastriques, hypertension, asthme, } \\
\text { antirhumastismale, cicatrisante. }\end{array}$ \\
\hline Borrago officinalis & Bourtrache (boukhrich) & propriété antitussive \\
\hline $\begin{array}{l}\text { Opuntia ficus- } \\
\text { indica }\end{array}$ & Figuier de Barbarie (hind) & $\begin{array}{l}\text {-fleurs: diurétique, lithiase urinaire } \\
\text {-racine: antidiabétique, } \\
\text {-frnits: antidiarréique }\end{array}$ \\
\hline Capparis spinosa & Caprier commun (kabbar) & $\begin{array}{l}\text { antirhumatismale, articulation douloureuse, } \\
\text { thume, sinusite, hypertension }\end{array}$ \\
\hline Salicornia arabica & Salicorne (hmadha) & eczema \\
\hline $\begin{array}{l}\text { Cistus } \\
\text { monspeliensis }\end{array}$ & $\begin{array}{l}\text { Ciste de Montpellier } \\
\text { (oumillia) }\end{array}$ & blessures extemes, dépuratif \\
\hline Bellis annua & $\begin{array}{l}\text { Paquerette annuelle } \\
\text { (oquouana) }\end{array}$ & $\begin{array}{l}\text {-dige: constipation } \\
\text {-Aleurs: aćrophagie, diabète, vermifuge, douleurs } \\
\text { dentaires }\end{array}$ \\
\hline Calendula arvensis & Souci des champs (karchoun) & chumatisme, fièvre, céphalée \\
\hline $\begin{array}{l}\text { Ecballium } \\
\text { elaterium }\end{array}$ & $\begin{array}{l}\text { Concombre d'âne (frquous } \\
\text { h'mir) }\end{array}$ & ictère par inhalation, migraine, acné \\
\hline
\end{tabular}




\begin{tabular}{|c|c|c|}
\hline $\begin{array}{c}\text { Nom } \\
\text { Scientifique }\end{array}$ & Nom Français/ Arabe & Utilisation \\
\hline Jasminus trutican & Jasmin jaune (boulla & usage exteme contre la gales \\
\hline Olea europea & Olivier (zitoun) & $\begin{array}{l}\text { constipation, ictère, antirussive, tuberculose, } \\
\text { grippes, hypotensives, orite... }\end{array}$ \\
\hline Clematis flammula & $\begin{array}{l}\text { Clématite brülante (nar } \\
\text { berda) }\end{array}$ & thumatisme, eczéma \\
\hline Nigella damascena & $\begin{array}{l}\text { Nigelle de Damas (habba } \\
\text { souda) }\end{array}$ & $\begin{array}{l}\text { analeprique cardiaque, antirhumatismale, } \\
\text { antiulcéreux }\end{array}$ \\
\hline Zizyphus lonus & Jujubier sauvage (nbeg) & propriété pectorales, leucomes oculaires \\
\hline $\begin{array}{l}\text { Crataegus azarolus } \\
\text { nas }\end{array}$ & Azérolier (zaarour) & antidiabètique, angine \\
\hline Verbena officinallis & verveine (tronjia) & tranquilisante, colique \\
\hline $\begin{array}{l}\text { Juniperus } \\
\text { phoenicea }\end{array}$ & Genevrier de phoenicie (äriar) & $\begin{array}{l}\text { antirhumatismale, antidiabètique, } \\
\text { antidianchéique... }\end{array}$ \\
\hline Malva sylvestris & Grande mauve (khobiza) & $\begin{array}{l}\text { inflammation oculaires, piqüres d'insectes, } \\
\text { constipation }\end{array}$ \\
\hline Foeniculum vulgare & Fenouil (besbes) & $\begin{array}{l}\text { diarrhées, gastralgies, dysurie, lactation, ulcère } \\
\text { gastrique... }\end{array}$ \\
\hline Ceratoniz siliquz & Caroubier (charroub) & $\begin{array}{l}\text { antidiarrhéique, antidiabétique, maladies } \\
\text { cardiaques, }\end{array}$ \\
\hline Nerium oleander & Laurier rose (khadhraya) & $\begin{array}{l}\text { strictement en usage extetne: eczéma, morsure } \\
\text { de chien, insolation, douleurs dentaires, } \\
\text { hémiplégie }\end{array}$ \\
\hline Solanum nigrum & Morelle noire (tmatem kleb) & brîutures et dermatose, eczéma, érisypele \\
\hline $\begin{array}{l}\text { Solanum } \\
\text { sodomeum }\end{array}$ & $\begin{array}{l}\text { Pomme de Sodome (lim } \\
\text { nsara) }\end{array}$ & $\begin{array}{l}\text { en cataplasme pour les verrues et en friction } \\
\text { pour l'eczéma }\end{array}$ \\
\hline Urtica pilulifera & Orties ì pilule (horriqua) & engechures, anurie, énurésie et lithiases thénales \\
\hline $\begin{array}{l}\text { Chenopodium } \\
\text { mumale }\end{array}$ & Anserine des murs (mazrita) & maladie asthénique et fievre \\
\hline
\end{tabular}

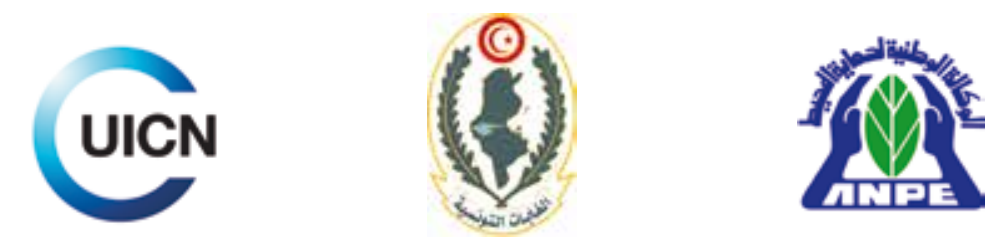

Dans le cadre d'un projet de recherche, la Direction Générale des Forêts (DGF), l'Agence Nationale de la Protection de l'Environnement (ANPE) en collaboration avec l'Union Internationale pour la Conservation de la Nature (UICN) réalisent une étude sur

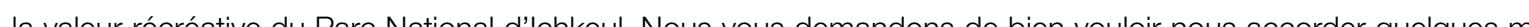

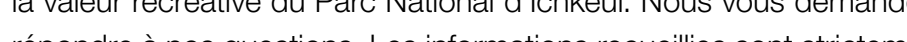
répondreà nos questions. Les informations recueilles sont stictement confidentielles et seront tratíes d'une façon anonyme.

1. Est-ce que c'est votre première visite au parc ? पOui घNon

2. Si non, combien de fois avez-vous visitez ce site durant les 12 derniers mois? fois

3. Connaissez-vous d'autres espaces naturels ? : $\square$ Oui $\square$ Non,

4. Si oui, lesquels?

5. Combien de temps comptez-vous passer sur le site ? : ..............mn

6. Quelles sont les activités que vous réalisez sur le site et qui motivent votre visite?

\begin{tabular}{|l|l|l|}
\hline Motifs de la visite & Détails & Classification \\
\hline 1. Découverte nature paysage & & \\
\hline 2. Observation des oiseaux, buffles, autres & & \\
\hline 3. Récréation, Calme, tranquillité Pique-nique & & \\
\hline 4. Etude, projet scientifique & & \\
\hline 5. Services liés au Hammam & & \\
\hline 6. Autres... & & \\
\hline
\end{tabular}

7. De quelle Ville avez-vous fait votre départ aujourd'hui (+Gouvernorat)?

8. Êtes-vous résident en Tunisie ? पOui (passez à la question 13) पNon

9. Sinon; Est-ce que la visite à lchkeul est la principale raison de votre visite en Tunisie ?
$\square$ Oui $\square$ Non, Si non (passez à la question 13)
10. Combien vous a coûté votre voyage depuis votre pays de résidence jusqu'en Tunisie ? (ticket d'avion, ticket de train,
bus, location de voiture, frais de carburant, réservation, etc.).......
11. Durée totale de séjour en Tunisie? .....................Jours
12. Aviez-vous une idée sur Le Parc National d'I'chkeul avant votre voyage en Tunisie?
$\square$ Oui $\square$ Non


13. Allez-vous faire l'aller-retour depuis votre résidence principale (lieu de séjour en Tunisie pour les non-résidents) à ce site dans la journée? GOui (passez à la question 18) 口Non,

14. Sinon; Est-ce que la visite à Ichkeul est la principale raison de votre visite dans la région? 口Oui $\square$ Non, Si non (passez à la question $\mathbf{1 8}$

15. Au cours de ce séjour, combien de nuitées allez-vous passer dans la région?..Nuitées

16. Au cours de ce séjour combien de fois allez-vous venir sur ce site? .................fois

17. Dans le cas où l'hébergement est payant, combien payez-vous par personne/nuitée ? ...DT

18. Quelle distance en kilomètres cela représente-t-il ? . Km

19. Par quel moyen de transport, principalement, êtes-vous venu aujourd'hui sur ce site?

\begin{tabular}{|l|l|l|l|l|l|}
\hline & $\begin{array}{l}\text { Cons. } \\
\text { //100 km }\end{array}$ & $\begin{array}{l}\text { Type de } \\
\text { carburant }\end{array}$ & $\begin{array}{l}\text { Cout de } \\
\text { location DT/j }\end{array}$ & $\begin{array}{l}\text { Nombre } \\
\text { adultes }\end{array}$ & $\begin{array}{l}\text { Nombre } \\
\text { enfants (-12) }\end{array}$ \\
\hline Voiture part. & & & & & \\
\hline Voiture de loc. & & & & & \\
\hline Voyage organisé & & & & & \\
\hline Autres (préciser) & & & & & \\
\hline
\end{tabular}

20. Pouvez-vous situer votre degré de satisfaction de votre visite sur une échelle de 1 à 10 ? :

21. Pensez-vous retourner visiter ce site ? : $\square$ Oui $\square$ Non

Si un droit d'entrée devait être appliqué pour l'accès au site, seriez-vous disposés à payer ? :

22. Si oui, quelle est la somme maximale que vous êtes prêt à payer? :

口Pour un Adulte

$\square$ Pour un Enfant (-12ans)

23. Si non, Pourquoi ?

$\square$ Ce n'est pas à moi de payer $\quad$ 口Il n'est pas nécessaire de préserver cet espace

$\square$ Mes moyens financiers ne me le permettent pas

$\square$ Je n'ai pas assez d'informations pour me decider

$\square$ Je crains de payer pour les autres $₫$ Je ne me sens pas concerne

$\square$ Autres raisons..........

24. Situer votre âge selon ces intervalles:

$\square 35$ à 49 ans $\quad \square 50$ à 64 ans

$\square<5$ ans

25. Adresse de résidence : Ville : ........................ Pays.............

26. Nationalité :

27. Sexe : पMasculin $\square$ Féminin

28. Situation familiale : $\square$ Célibataire $\square$ marié(e) $\square$ autre :

29. Niveau d'instruction: $\square$ Non $\square$ Primaire $\square$ Secondaire $\square$ Supérieur

30. Profession

$\square$ Retraités पEtudiant/ lélève $\square$ Cadre supérieur $\square$ Profession libérale $\square$ Commerçant $\square$ Ouvrier Dautres

31. Pouvez-vous situer votre revenu annuel parmi les réponses proposées (en dinars tunisiens) $\square<1500 \quad \square 1500$ à $2000 \quad \square 2000$ à $5000 \quad \square 5000$ à 10.000 10.000 à $20.000 \quad \square 20.000$ à $50.000 \quad \square>50.000$

\section{Merci pour votre collaboration}


ANNEXE 4 : QUESTIONNAIRE SUR LA VALEUR DE L'AMÉLIORATION DE LA BIODIVERSITÉ

DU PARC NATIONAL DE L'ICHKEUL - TUNISIE HTTPS : //GOO.GL/KDDM7B

\section{Préambule}

L'administration tunisienne, en collaboration avec l'Union Internationale pour la Conservation de la Nature (UICN) et l'appui de WWF, réalise une étude sur la valeur de l'amélioration de la biodiversité du Parc National d'Ichkeul. Nous vous deman-

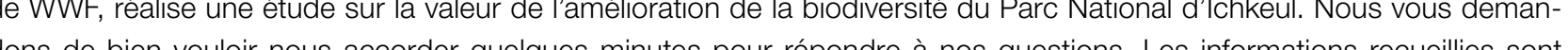
dons de bien vounir strictement confientiles et seront trantés dune

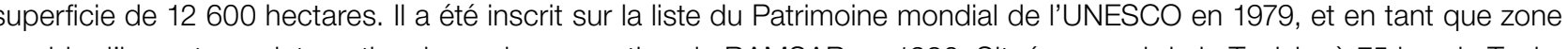
humide d'importance internationale par la convention de RAMSAR en 1980. Situe au nord de la Tunisie, a $75 \mathrm{~km}$ de Tunis, il est composé d'un lac ( $8600 \mathrm{ha}$ ), des marais (2700 ha) et d'un massif montagneux (1300 ha). II abrite des milliers d'oiseaux migrateurs comme des oles cendrees, des canards siffleurs, des foulques, des cigognes. Passereaux, rapaces, reptiles, tortues et buffles ont également élu domicile dans ce parc. La flore du parc est très variée. On y trouve plus de 500 espèces de plantes. Pour plus d'informations : consultezhttp : //whc.unesco.org/fr/list/8.

Le Parc subit des risques de degradation de la biodiversite du parc, notamment durant les annees de secheresse lorsque le lac n'est pas assez alimenté par les eaux des barrages, aussi, certaines ressources sont surexploitees par la population locale. Pour cela, l'administration du parc projette l'élaboration d'un programme visant l'amélioration de la biodiversité de l'écosystème de l'chkeul, avec ses trois sous-écosystèmes (lac, marais et montagne). Le programme comporte trois composantes (i) la protection durable de l'écosystème lac-montagne, (ii) le développement socio-économique de la périphérie et (iii) un système de gestion de l'eau efficace du lac.

Le montant qui sera alloué à ce programme dépendra étroitement de la disposition à payer des adhérents à ce programme. L'amélioration de la biodiversité qui en découle dépendra du montant collecté.

e payement sera annuel pour tous les volontaires par versement sous forme de donations. Le montant sera versé dans un compte spécial géré, selon la réglementation en vigueur par une ONG environnementale pour financer exclusivement le programme envisagé. Considérez aussi que votre revenu peut supporter le payement consenti. Merci de répondre aux questions suivantes:
*Obligatoire $\square$ Oui

$\square$ Non

Si non, avez-vous l'intention de le visiter?

पOui

3. Qu'est-ce que vous pensez de ce programme ? $\square$ Très défavorable

$\square$ défavorable

IIndifferent

口Favorable

口Très favorable

$\square 18$ à 24 ans

$\square 25$ à 34 ans

$\square 35$ à 49 ans

10. Pays de résidence : *

11. Nationalité :

12. Sexe : *

$\square$ Masculin

口Féminin

\section{Seriez-vous disposé(e) à payer ? *}

$\square$ Oui

$\square$ Non

13. Profession :

पEtudiant

$\square$ Cadre supérieur

$\square$ Profession libérale

5. Quel est le montant maximum par personne (adulte) que vous etes dispose(e)s a payer annuellement pour ce programme ? en DT (Dinars tunisiens) *

$1 \mathrm{DT}=0,5$ Euro environ ; N.B. : Veuillez mettre 0 si vous n'êtes pas disposés à payer

\section{Pour quel type
पMontagne
$\square$ Lac}

7. Veuillez préciser l'espèce sauvage (animale, végétale)

pour laquelle vous souhaiteriez une amélioration ?

Employe $(e)$

14. Situation familiale : *

$\square$ Célibataire

口Marié(e)

$\square$ Autre :

15. Pouvez-vous situer votre revenu annuel selon ces intervalles (en dinars tunisiens) ?

DT $=0,5$ euros environ

$\square<1500$

8. Si vous n'êtes pas disposé(e)s à payer, quelles son les raisons?

$\square$ Ce n'est pas à moi de payer

$\square$ Je ne paye pas pour un service «naturel" -Don de Dieu-

$\square$ Mes moyens financiers ne me le permettent pas

$\square$ Je n'ai pas assez d'informations pour me décider

$\square$ Je crains de payer alors que d'autres en bénéficient

$\square$ Je ne me sens pas concerné(e)

$\square 1500$ a 2000

ㅁ2000 à 5000

$\square 5000$ à 10.000

ㅁ10.000 à 20.000

$\square$
$\square>50.000$

Autre:

16. Autres commentaires 


\section{Aménagement}

- Toutes les ZPI au niveau du Djebel et des marais sont protégées par une clôture métallique.

a

des marais pour la restauration des zon eas d'ou

Aménagement de l'entrée principale du parc et d'un portique sur la route d'accès au parc portant

les logos du parc et des trois conventions internationales dans lesquelles il est inscrit.

Acquisition et pose de panneaux de signalisation aux endroits stratégiques

- Asphaltage de 6,2 km (de Hammam Bel Abbes à douar Chardoudl).

d'une table d'orientation pres de l'ecomusee.

Ouverture de $8 \mathrm{~km}$ de tranchée pare feu le long de la crête E-W du Djebe.

Ouverture des sentiers pédestres.

Mesures des débits des sources et analyses bactériologiques des eaux réalisées par les agents de l'office

national du thermalisme. Une convention entre le CRDA de Bizerte et l'office national du thermalisme portan

sur la réhabilitation des hammams et leur mode d'exploitation futur. Travaux de captage des sources.
Tous les bâtiments existants ont été réhabilités. Le musée géologique a été créé et équipé

Te

des anciennes carrières pour la production des tableaux en mosä̈que.

Les falaises des carrières constituent des refuges pour certains rapaces et pigeons.

Une prairie permanente sur 122 ha a été installée. Elle est exploitée en pâturage par le cheptel des éleveurs locaux.

Deux miradors en bois ont été installés pour l'observation des oiseaux d'eau.

La grotte est fermée par une porte. L'entrée à la grotte est contrôlée par le conservateur du parc.

Des visites assistées sont réservées aux scientifiques, chercheurs et spéléologues.

-Réactualisation des panneaux didactiques.

-Renforcement des panneaux solaires et remplacement des batteries accumulatrices.

Rénovation complète des sanitaires

.

- Installation du lecom

.

\section{Equipement}

1 véhicule de liaison (4×4)

- 10 mobylettes.

1 embarcation

5 tracteur et une remorque.

Equipement pour écogardes.

- Matériel d'observation et d'enregistrement.

- Matériel audio-visuel.

- Materiel de bureau (ordinateurs + PC, imprimante et scanner, photocopieuse....

- Outillage divers

\section{Matériel publicitaire}

- Pose de panneaux signaletiques aux endroits strategiques de I'Unité et au niveau des agglomérations avoisinantes.

Edition de la brochure réactualisée en couleurs sur le PNI.

- Visites guidees decollers et d'etudiants.

Atlas des parcs nationaux et reser

res naturelles MEDD.

20ème anniversaire de l'ANPE (organisation d'atelier sur le parc national d'Ichkeul) ANPE

Publication d'un document intitulé :

«Les 20 sites Ramsar de Tunisie : Zones Humides d'Importance Internationale „ DGF.

Publication de document sur l'écotourisme (au Nord, au Centre et au Sud Tunisien) DGF/GTZ.

Organisation communautaire (GDA/lchkeul).

- Formation des membres du GDA par une ONG nationale «ASAD » dans

- Organisation d'un atelier de conce entann 2007 et 2008

(2) partenaires-

\section{Projets pilotes et aspect financier}

- Vulgarisation et démarrage de certains projets pilotes « porteurs " et soutenus par

la population (apiculture, páturage ameliore, greffage des oleastres).

-

au niveau de la périphérie

- Intégration du parc dans les circuits touristiques de certains tours opérateurs.

Sources : CRDA Bizerte 2009 ; MARH/MEDD. 2008 


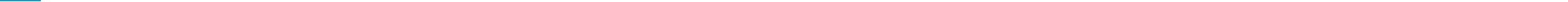




\section{UICN}

\section{UNION INTERNATIONALE POUR}

LA CONSERVATION DE LA NATURE

CENTRE DE COOPÉRATION

POUR LA MÉDITERRANÉE (UNICN-MED)

C/ Marie Curie 22 - Andalucia Science and Technology Park 29590 Campanillas

Malaga, Espagne

Tél +34 352028430

Fax +34 352028430

uicnmed@iucn.org

\section{www.iucn.org/mediterranee}

Le Centre de Coppération pour la Méditerranée de l'UICN est soutenu par :

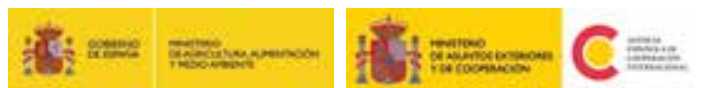

